\title{
7. THE PALEOMAGNETISM OF SEDIMENTS ACQUIRED FROM THE GOBAN SPUR ON DEEP SEA DRILLING PROJECT LEG 801
}

\author{
Hilary A. Townsend, Oceanography Department, Southampton University²
}

\begin{abstract}
Paleomagnetic results from sediments acquired from the continental margin at DSDP Sites 548, 549, 550, and 551 are described. Where possible, the results were used to construct a polarity reversal stratigraphy for the sections sampled, thus enabling the biostratigraphic dating of the sediments to be refined. Several sections in this study were found to be suitable for magnetostratigraphic work, in particular the upper Paleocene to middle Eocene sediments from Site 549 , which contained rich faunal assemblages. These sediments are underlain by a thick sequence of Cretaceous sediments that formed during the Long Cretaceous normal polarity interval. Sediments that formed during the later part of this magnetically quiet interval were also recovered at Site 550 . Three short reverse polarity intervals were also recovered at this site; they lie directly over basement and are thought to represent a mixed-polarity interval of late Albian age. They may therefore provide important evidence concerning the age of the earliest sediments at this site.

In addition, measurements of the magnetic susceptibility and intensity of remanent magnetism proved to be of interest. A significant decrease in the susceptibility and intensity values close to the early/middle Eocene boundary was noted at Sites 548 and 549. This decrease may be correlated with the results from Holes 400A and 401, which were drilled on DSDP Leg 48 in the northeast Bay of Biscay. The decrease may represent an abrupt reduction in the supply of terrigenous material at the end of the early Eocene, reflecting, perhaps, a change in sediment transport processes at that time.
\end{abstract}

\section{INTRODUCTION}

This paper describes the results of paleomagnetic studies carried out on the sediments recovered from four sites drilled along a transect across the northern Biscay continental margin during Leg 80 of the Deep Sea Drilling Project. The main aim of this study was to construct a magnetic stratigraphy that would complement the biostratigraphic studies and provide additional information on the geological ages of the sediments drilled and the dating of the major paleoceanographic events recorded in the sediments.

\section{PALEOMAGNETIC TECHNIQUES}

\section{Sampling Procedures}

Paleomagnetic samples were taken from the relatively soft sediments by using $2.5-\mathrm{cm}$ plastic cylinders that were then sealed with cellophane to prevent the sediment from drying out. In the case of the consolidated sediments, $2.5-\mathrm{cm}$-diameter cylinders were drilled with a diamond-tipped core drill. In both cases an uphole orientation line was carefully marked on the samples before they were removed from the core.

\section{Measuring Procedures}

Most of the measurements of direction and intensity of remanence were carried out on a standard Digico spinner magnetometer (Molyneux, 1971) installed on the Glomar Challenger. The samples were incrementally demag-

\footnotetext{
${ }^{1}$ Graciansky, P. C. de, Poag, C. W., et al., Init. Repts. DSDP, 80: Washington (U.S Govt, Printing Office) dom.
}

netized in an alternating field (af) by using a Schonstedt single-axis demagnetizer. Subsequent measurements were completed after the cruise in the paleomagnetic laboratory at Southampton University. Throughout the period of the cruise the average noise level of the shipboard magnetometer was $0.05 \mu \mathrm{G}$.

At Sites 548 and 549 the variable length hydraulic piston corer (VLHPC) was used for the first time; a method for orienting the cores was also tested at these two sites. Knowledge of the orientation of a core would permit the absolute azimuth of the core to be measured so that the assignment of polarity could be based on both the declination and the inclination of stable remanent magnetization rather than inclination only. Unfortunately, as a result of technical difficulties fewer than half the cores could be oriented at these sites, so the new facility was of limited value on Leg 80 . However, the additional paleomagnetic information available from having oriented IPOD cores should prove to be useful in the future.

Figure 1 shows the stratigraphic variation of declination and inclination for the upper $80 \mathrm{~m}$ at Site 548 , where the absolute azimuth was successfully recorded in a number of cores. After magnetic cleaning, the oriented cores generally possessed a northerly paleomagnetic declination $\left(360 \pm 20^{\circ}\right)$ and a positive inclination, characteristics indicative of a normal polarity stable direction. A single oriented sample at $72 \mathrm{~m}$ yielded a shallow negative inclination and a more southerly declination, which suggests that a short magnetic event occurred at this point in the Brunhes normal polarity epoch.

Figure 1 shows one of only a few short intervals where the azimuth of the core was recorded successfully, however. In most of this study the assignment of the magnetic polarity is based on the variation in the inclination 

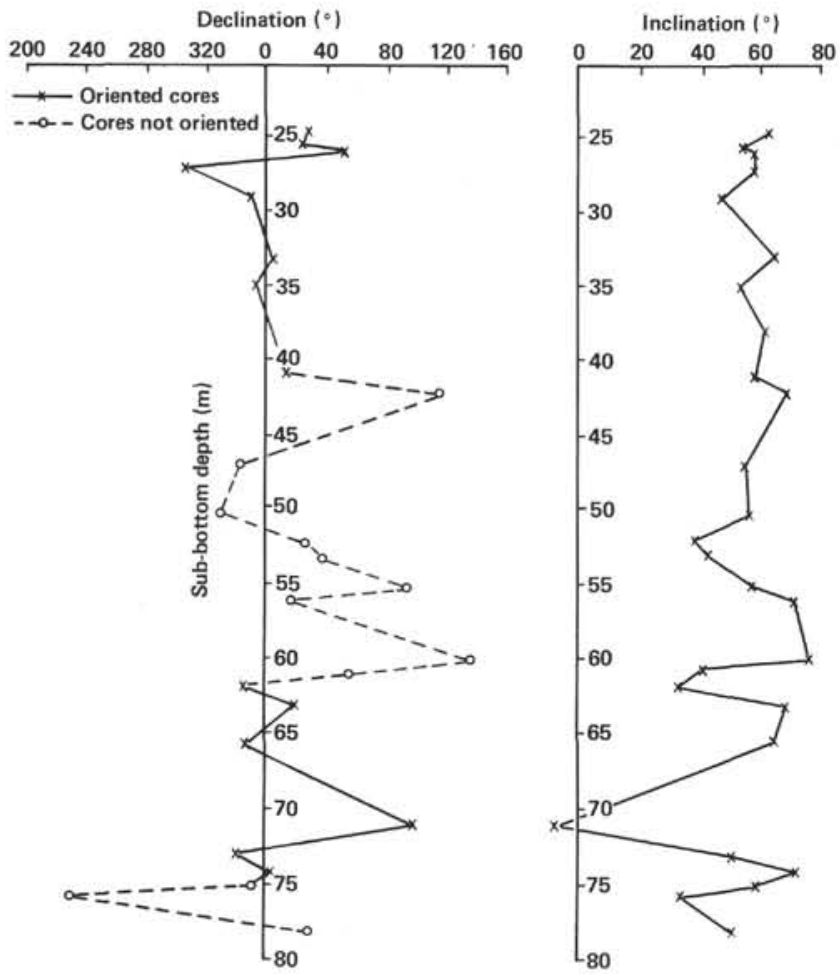

Figure 1. Downhole variation of declination and inclination at Hole 548.

of the stable remanent magnetization alone. Since the sites are situated in the Northern Hemisphere, positive inclinations are taken to signify normal polarity and negative inclinations reverse polarity.

\section{RESULTS}

\section{Site 548}

Site 548, which was the shallowest site in the Goban Spur transect, was drilled on the seaward edge of a tilted block of Hercynian basement. Two holes were drilled at this site, Hole 548 with the VLHPC and Hole 548A with the conventional rotary drill.

\section{Hole 548}

Recovery from Hole 548 was extremely good, and approximately 200 oriented samples from the Pleistocene and Pliocene sediments were taken. Each sample was demagnetized in an alternating field until the polarity of the stable component was considered unequivocal. The more strongly magnetized samples were demagnetized at peak fields up to 200 or 300 Oe; the intensity of the more weakly magnetized samples became too weak for reliable measurement after demagnetization at fields greater than 75 or $100 \mathrm{Oe}$. The results from this hole are summarized in Table 1. Figure 2 illustrates the behavior of four samples from this hole on demagnetization. Two of the samples (548-10-4, 6-8 cm and 548-1-1, 14-43 $\mathrm{cm}$ ) are moderately stable and have been assigned a normal polarity. The other two are reverse polarity samples. One of them (Sample 548-10-5, 6-8 cm) has a reasonably high stability, whereas the other (Sample 548-10-5,
$84-86 \mathrm{~cm}$ ) has a shallow initial negative inclination that becomes steeper on demagnetization, suggesting that a secondary component is being removed during the demagnetization process.

\section{Magnetic Polarity Record}

Figure 3 shows the stratigraphic variation of magnetic inclination after optimum demagnetization, together with the inferred polarity reversal sequence and the assigned anomaly numbers. The biostratigraphic zones established (Müller, this volume and Snyder, this volume) have also been included in the figure. In the upper section there is a long normal polarity interval (down to $84 \mathrm{~m}$ sub-bottom) that contains evidence of a possible magnetic field excursion. Below this interval there are two well-defined normal polarity intervals that are thought to represent the Jaramillo and Olduvai events. The Olduvai Event spans the Pleistocene/Pliocene boundary, which has been placed at approximately $102 \mathrm{~m}$ subbottom on nannofossil evidence (Müller, this volume). In the Pliocene sediments a further sequence of polarity reversals is evident. They are not as well defined as the later reversals, but they probably correspond to the sequence of polarity changes that together represent Anomalies $2 \mathrm{~A}$ and 3 .

\section{NRM Intensity and Volume Susceptibility}

Figure 4 shows the downhole variation of NRM intensity and volume susceptibility. The correlation of the two plots is good, suggesting that the NRM intensity reflects magnetic mineral content instead of variations in the intensity of the geomagnetic field at the time of deposition. The abrupt decrease in intensity and susceptibility at approximately $59 \mathrm{~m}$ sub-bottom corresponds to the lithologic change between Subunits 1a and 1b, which is distinguished by a downward decrease in terrigenous material and an increase in calcium carbonate. The minor fluctuations in NRM intensity and volume susceptibility also seem to correspond to small variations in the amount of marly material.

\section{Hole 548A}

Approximately 220 samples were taken from Hole $548 \mathrm{~A}$, and the paleomagnetic results are summarized in Table 2. The results from the upper part of the hole (down to $380 \mathrm{~m}$ sub-bottom) are difficult to interpret because the sequence is condensed in parts. In addition, the frequency of magnetic field reversals was relatively high during the Miocene and Oligocene epochs. Inasmuch as the sediments from this section are also very weakly magnetized $(0.03-0.20 \mu \mathrm{G})$, no conclusive magnetic polarity reversal sequence can be defined.

The samples below $380 \mathrm{~m}$ sub-bottom have considerably higher NRM intensities $(0.20-50.0 \mu \mathrm{G})$, and most were demagnetized in peak fields up to 200 or 300 Oe. Figure 5 illustrates the behavior of a typical normal polarity sample $(548 \mathrm{~A}-33-2,107-109 \mathrm{~cm})$ and a reverse polarity sample (548A-33-1, 106-108 cm). The magnetization direction of the normal polarity sample is moderately stable; the reverse polarity sample shows a considerable change between the NRM and 100 Oe steps but little 
Table 1. Paleomagnetic results, Hole 548.

\begin{tabular}{|c|c|c|c|c|c|c|}
\hline & Sub-bottom & & $\begin{array}{c}\text { Before } \\
\text { lagnetiza }\end{array}$ & & Demagnetization & \\
\hline $\begin{array}{c}\text { Core-Section } \\
\text { (interval in cm) }\end{array}$ & $\begin{array}{l}\text { depth } \\
\text { (m) }\end{array}$ & $\begin{array}{l}\text { D } \\
\left({ }^{\circ}\right)\end{array}$ & $\begin{array}{c}1 \\
\left({ }^{\circ}\right)\end{array}$ & $\begin{array}{l}\text { Int } \\
(\mu \mathrm{G})\end{array}$ & $\begin{array}{l}\text { field } \\
\text { (Oe) }\end{array}$ & $\begin{array}{c}D \\
\left({ }^{\circ}\right)\end{array}$ \\
\hline $1-1,41-43$ & 0.41 & 108.0 & 64.3 & 27.82 & 300 & 120.6 \\
\hline $1-2,106-108$ & 2.56 & 85.1 & 64.0 & 12.88 & 300 & 122.5 \\
\hline $2-1,57-59$ & 4.57 & 338.9 & 55.0 & 12.52 & 200 & 330.9 \\
\hline $2-2,122-124$ & 6.72 & 23.5 & 47.2 & 6.02 & 200 & 22.0 \\
\hline $2-3,82-84$ & 7.82 & 172.5 & 83.0 & 11.11 & 200 & 181.9 \\
\hline $2-4,82-84$ & 9.32 & 320.0 & 56.2 & 13.56 & 200 & 297.5 \\
\hline $2-5,19-21$ & 10.19 & 163.2 & 62.2 & 22.53 & 200 & 157.5 \\
\hline $2-6,27-29$ & 11.79 & 263.9 & 79.7 & 21.75 & 200 & 228.4 \\
\hline $2-7,20-22$ & 13.20 & 311.0 & 72.7 & 20.74 & 200 & 284.5 \\
\hline $3-1,23-25$ & 13.73 & 108.1 & 56.3 & 10.52 & 200 & 116.1 \\
\hline $3-2,45-47$ & 15.47 & 330.5 & 57.2 & 18.26 & 200 & 217.5 \\
\hline $3-3,112-114$ & 17.62 & 208.2 & 60.7 & 11.69 & 200 & 209.5 \\
\hline $3-4,37-39$ & 18.37 & 40.4 & 70.4 & 12.02 & 200 & 45.5 \\
\hline $3-5,25-27$ & 19.75 & 56.6 & 54.9 & 16.06 & 200 & 61.6 \\
\hline $3-6,35-5$ & 21.35 & 10.9 & 64.6 & 10.83 & 200 & 16.7 \\
\hline $3-7,25-27$ & 22.75 & 215.1 & 65.0 & 10.26 & 200 & 211.0 \\
\hline $4-1,103-104$ & 24.02 & 9.2 & 64.7 & 18.21 & 200 & 26.4 \\
\hline $4-2,103-105$ & 25.53 & 2.7 & 66.8 & 10.65 & 200 & 25.9 \\
\hline $4-3,48-50$ & 26.48 & 39.0 & 64.4 & 19.72 & 200 & 54.7 \\
\hline $4-4,49-51$ & 27.99 & 301.6 & 59.6 & 29.68 & 200 & 302.5 \\
\hline $4-5,49-51$ & 29.49 & 338.5 & 50.7 & 21.27 & 200 & 349.8 \\
\hline $4-6,39-41$ & 30.89 & 12.9 & -82.6 & 25.34 & 200 & 73.1 \\
\hline $5-1,117-119$ & 33.67 & 327.3 & 66.0 & 20.84 & 200 & 1.4 \\
\hline $5-3,38-40$ & 35.88 & 343.3 & 57.5 & 24.41 & 200 & 354.7 \\
\hline $5-5,36-38$ & 38.86 & 341.7 & 59.2 & 15.39 & 200 & 3.8 \\
\hline $5-7,33-35$ & 41.83 & 8.8 & 57.4 & 16.45 & 200 & 12.9 \\
\hline $6-2,43-45$ & 42.43 & 124.3 & 79.7 & 22.19 & 200 & 114.1 \\
\hline $6-4,109-111$ & 47.59 & 341.6 & 55.6 & 18.97 & 200 & 341.4 \\
\hline $6-6,107-109$ & 50.57 & 330.3 & 55.8 & 18.41 & 200 & 333.0 \\
\hline $7-1,95-97$ & 52.45 & 359 & 42.7 & 14.86 & 200 & 35.8 \\
\hline $7-2,18-20$ & 53.18 & 42.3 & 59.3 & 11.26 & 200 & 49.6 \\
\hline $7-3,102-104$ & 55.52 & 82.9 & 72.2 & 9.04 & 200 & 94.7 \\
\hline $7-4,15-17$ & 56.15 & 359.9 & 77.1 & 23.09 & 200 & 16.1 \\
\hline $7-6,120-122$ & 60.20 & 252.5 & 87.6 & 0.09 & 50 & 139.3 \\
\hline $7-7.30-32$ & 60.80 & 27.5 & 54.7 & 0.61 & 50 & 56.5 \\
\hline $8-1,50-52$ & 61.50 & 355.5 & 40.8 & 0.58 & 50 & 346.5 \\
\hline $8-2,51-53$ & 63.01 & 18.4 & 74.7 & 0.76 & 75 & 23.5 \\
\hline $8-3,50-52$ & 64.50 & 180.2 & 67.1 & 1.38 & 100 & 156.9 \\
\hline $8-4,50-52$ & 66.0 & 327.4 & 78.3 & 8.836 & 100 & 347.5 \\
\hline $8-6,31-33$ & 68.81 & 109.6 & 49.6 & 0.49 & 100 & 127.0 \\
\hline $9-1,98-100$ & 71.48 & 99.9 & -9.0 & 0.35 & 150 & 169.7 \\
\hline $9-3,29-31$ & 73.29 & 305.5 & 65.2 & 0.30 & 100 & 340.5 \\
\hline $10-2,103-105$ & & 211.5 & 67.8 & 4.30 & 200 & 7.3 \\
\hline $10-3,103-105$ & & 348.0 & 58.6 & 10.07 & 200 & 349.5 \\
\hline $10-4,6-8$ & 75.06 & 269.3 & 59.4 & 5.55 & 200 & 279.1 \\
\hline $10-4,84-86$ & 75.84 & 254.8 & 37.1 & 4.18 & 300 & 223.2 \\
\hline $10-5,6-8$ & 76.56 & 31.6 & -75.2 & 7.522 & 200 & 67.8 \\
\hline $10-5,84-86$ & 77.34 & 329.7 & -14.6 & 1.24 & 200 & 326.6 \\
\hline $10-6,6-8$ & 78.06 & 54.4 & 71.1 & 1.90 & 200 & 48.1 \\
\hline $10-6,84-86$ & 78.84 & 10.1 & 51.7 & 0.363 & 50 & 33,1 \\
\hline $11-1,33-36$ & 80.33 & 337.9 & 38.1 & 0.19 & 150 & 303.0 \\
\hline $11-1,101-103$ & 81.01 & 306.7 & -13.9 & 0.23 & 150 & 309.1 \\
\hline $11-3,101-103$ & 84.1 & 211.5 & 82.5 & 5.32 & 200 & 225.1 \\
\hline $11-4,33-36$ & 84.83 & 227.4 & 24.7 & 1.61 & 200 & 249.7 \\
\hline $11-4,101-103$ & 85.51 & 305.2 & -12.0 & 0.35 & 150 & 312.9 \\
\hline $11-5,33-36$ & 86.33 & 241.0 & 26.7 & 0.23 & 150 & 105.4 \\
\hline $11-5,101-103$ & 87.01 & 237.6 & -47.1 & 0.35 & 50 & 271.3 \\
\hline $11-6,33-36$ & 87.33 & 250.0 & 16.7 & 0.14 & 100 & 328.1 \\
\hline $12-1,138-140$ & 90.88 & 65.1 & 68.9 & 0.44 & 200 & 221.6 \\
\hline $13-1,112-114$ & 93.12 & 253.4 & 7.8 & 0.16 & 50 & 1.7 \\
\hline $13-2,106-108$ & 94.56 & 187.9 & 30.2 & 0.23 & 150 & 140.2 \\
\hline $13-2,116-118$ & 94.66 & 282.2 & 62.3 & 0.16 & 35 & 224.5 \\
\hline $13-3,16-18$ & 95.16 & 310.5 & 0.3 & 0.30 & so & 317.5 \\
\hline $13-3,97-99$ & 95.97 & 116.2 & 2.0 & 0.33 & 150 & 154.5 \\
\hline $13-4,16-18$ & 96.66 & 258.1 & 34.1 & 0.07 & 100 & 320.4 \\
\hline $13-4,112-114$ & 97.62 & 154.6 & -19.7 & -.19 & 150 & 128.8 \\
\hline $13-5,16-18$ & 95.16 & 261.8 & -3.2 & 0.52 & 150 & 267.5 \\
\hline $13-5,106-110$ & 99.08 & 245.2 & -19.2 & 0.16 & 150 & 157.8 \\
\hline $13-5,112-114$ & 99.12 & 279.3 & 32.4 & 0.41 & 100 & 333.1 \\
\hline $13-6,16-18$ & 99.66 & 215,8 & 35.2 & 0.31 & 150 & 233.0 \\
\hline $15-1,110-112$ & 101.1 & 357.9 & 34.2 & 0.13 & 100 & 357.4 \\
\hline $15-2,12-14$ & 101.62 & 295.4 & 51.8 & 0.24 & 150 & 300.4 \\
\hline $15-4,12-14$ & 104.62 & 253.8 & 13.4 & 0.26 & 150 & 259.5 \\
\hline $15-4,110-112$ & 105.73 & 301.2 & 60.7 & 0.37 & 100 & 353.1 \\
\hline $15-5,110-112$ & 107.10 & 118.4 & 71.7 & 0.39 & 100 & 98.7 \\
\hline $15-6,12-14$ & 107.62 & 227.8 & 22.1 & 0.31 & so & 207.1 \\
\hline $15-7,12-14$ & 109.12 & 284.8 & -4.7 & 0.29 & 100 & 185.3 \\
\hline $16-1,110-112$ & 109.60 & 204.4 & 10.4 & 0.09 & 50 & 120.2 \\
\hline $16-2,110-112$ & 111.10 & 267.8 & 72.3 & 0.10 & 100 & 290.0 \\
\hline $16-4,110-112$ & 112.60 & 285.2 & -27.9 & 0.13 & 50 & 279.4 \\
\hline $16-4,110-112$ & 114.10 & 286.0 & 33.3 & 0.16 & 50 & 338.8 \\
\hline $16-6,28-30$ & 126.28 & 22.7 & 43.6 & 0.23 & 50 & 338.8 \\
\hline $19-1,61-64$ & 131.61 & 171.1 & 62.9 & 0.01 & NRM & \\
\hline $19-2,29-31$ & 132.79 & 274.2 & -15.8 & 0.06 & NRM & \\
\hline $19-3,30-33$ & 134.3 & 264.3 & 41.0 & 0.38 & 50 & 275.9 \\
\hline $19-4,6-9$ & 135.56 & 272.1 & 72.1 & 0.26 & 50 & 270.9 \\
\hline $20-1,23-25$ & 136.23 & 124.6 & 79.2 & 0.25 & 50 & 210.7 \\
\hline $20-2,23-25$ & 137.73 & 158.1 & 55.7 & 0.19 & 50 & 76.1 \\
\hline $21-1,42-44$ & 142.92 & 286.7 & 30.7 & 0.23 & 50 & 248.7 \\
\hline $21-3,45-47$ & 144.45 & 253.7 & 21.9 & 0.24 & 50 & 271.9 \\
\hline
\end{tabular}


Table 1. (Continued).

\begin{tabular}{|c|c|c|c|c|c|c|c|c|c|}
\hline \multirow[b]{2}{*}{$\begin{array}{c}\text { Core-Section } \\
\text { (interval in } \mathrm{cm} \text { ) }\end{array}$} & \multirow{2}{*}{$\begin{array}{l}\text { Sub-bottom } \\
\text { depth } \\
\text { (m) }\end{array}$} & \multicolumn{3}{|c|}{$\begin{array}{c}\text { Before } \\
\text { demagnetization }\end{array}$} & \multirow{2}{*}{$\begin{array}{l}\text { Demagnetization } \\
\text { field } \\
\text { (Oe) }\end{array}$} & \multicolumn{4}{|c|}{ After demagnetization } \\
\hline & & $\begin{array}{l}D \\
\left({ }^{\circ}\right)\end{array}$ & $\begin{array}{c}1 \\
\left({ }^{\circ}\right)\end{array}$ & $\underset{(\mu \mathrm{G})}{\text { Int }}$ & & $\begin{array}{c}D \\
\left({ }^{\circ}\right)\end{array}$ & $\begin{array}{c}1 \\
\left({ }^{\circ}\right)\end{array}$ & $\begin{array}{c}\text { Int } \\
(\mu \mathrm{G})\end{array}$ & Polarity \\
\hline $22-1,95-97$ & 146.95 & 223.5 & 80.7 & 0.18 & 50 & 172.4 & 49.5 & 0.12 & $\mathrm{~N}$ ? \\
\hline $22-2,32-35$ & 147.82 & 284.1 & 40.1 & 0.17 & 50 & 260.1 & 66.4 & 0.14 & $\mathrm{~N}$ \\
\hline $22-3,32-35$ & 149.32 & 332.9 & 38.3 & 0.13 & 50 & 343.4 & 27.3 & 0.50 & $\mathrm{~N}$ \\
\hline $24-1,108-110$ & 157.08 & 292.0 & 48.9 & 0.04 & NRM & & & & $\mathrm{N}$ ? \\
\hline $24-2,108-110$ & 158.58 & 236.6 & 9.3 & 0.09 & 25 & 243.7 & 61.0 & 0.15 & $\mathrm{~N}$ \\
\hline $24-3,49-51$ & 159.49 & 341.0 & 54.9 & 0.09 & NRM & & & & $\mathrm{N}$ \\
\hline $25-1,31-33$ & 156.31 & 250.2 & 17.9 & 0.16 & 25 & 239.3 & 61.3 & 0.07 & $\mathrm{~N}$ \\
\hline $25-2,31-33$ & 157.81 & 241.9 & 49.1 & 0.34 & 50 & 274.4 & 26.2 & 0.16 & $\mathrm{~N}$ \\
\hline $25-3,31-33$ & 159.31 & 238.8 & 37.7 & 0.17 & 50 & 222.7 & 50.1 & 0.11 & $\mathrm{~N}$ \\
\hline $25-1,46-48$ & 161.46 & 279.5 & 46.8 & 0.09 & 50 & 259.7 & 16.9 & 0.17 & $\mathrm{~N}$ \\
\hline $26-3,19-21$ & 164.19 & 215.0 & 22.6 & 0.17 & 50 & 61.4 & 64.4 & 0.11 & $\mathrm{~N}$ \\
\hline $26-4,19-21$ & 165.69 & 267.1 & -12.9 & 0.08 & 25 & 282.1 & -23.8 & 0.02 & $\mathbf{R}$ \\
\hline $27-1,123-125$ & 172.23 & 329.5 & 23.9 & 0.03 & NRM & & & & $\mathrm{N}$ ? \\
\hline $27-2,123-125$ & 173.73 & 52.1 & 11.7 & 0.007 & NRM & & & & $\mathrm{N}$ ? \\
\hline $27-3,123-125$ & 175.23 & 120.5 & 67.0 & 0.04 & NRM & & & & $\mathrm{N}$ ? \\
\hline $27-4,14-16$ & 175.63 & 151.1 & -13.9 & 0.018 & NRM & & & & $\mathrm{R}$ ? \\
\hline $28-1,40-43$ & 176.4 & 258.8 & 56.9 & 0.22 & 50 & 157.6 & 11.2 & 0.09 & $\mathrm{~N}$ ? \\
\hline $28-2,40-43$ & 177.90 & 234.5 & 72.6 & 0.10 & 25 & 159.3 & 57.0 & 0.17 & $\mathrm{~N}$ ? \\
\hline $28-2,110-112$ & 178.6 & 170.6 & 46.7 & 0.08 & 25 & 155.0 & 62.0 & 0.06 & $\mathrm{~N}$ \\
\hline $28-3,41-43$ & 179.41 & 170.2 & -21.2 & 0.26 & 25 & 161.6 & -46.7 & 0.19 & $\mathbf{R}$ \\
\hline $28-3,95-98$ & 179.95 & 92.1 & 6.3 & 0.08 & 25 & 114.9 & 29.6 & 0.04 & $\mathrm{~N}$ \\
\hline $28-4,20-23$ & 180.7 & 251.6 & 14.7 & 0.07 & 25 & 275.6 & 63.1 & 0.05 & $\mathrm{~N}$ \\
\hline $29-1,26-29$ & 181.29 & 203.7 & 45.0 & 0.14 & 100 & 200.2 & -31.1 & 0.07 & $\mathrm{R}$ ? \\
\hline $29-1,119-121$ & 182.19 & 265.9 & -5.0 & 0.03 & NRM & & & & $\mathrm{R}$ ? \\
\hline $29-2,33-35$ & 182.83 & 256.2 & 44.2 & 0.26 & 150 & 350.0 & -8.2 & 0.04 & $\mathrm{R}$ ? \\
\hline $29-2,118-120$ & 183.68 & 275.8 & 6.9 & 0.15 & 25 & 264.1 & 17.5 & 0.10 & $?$ \\
\hline $29-3,27-29$ & 184.27 & 223.8 & 33.8 & 0.16 & 100 & 245.4 & 0.2 & 0.06 & $\mathrm{R} ?$ ? \\
\hline $29-3,118-120$ & 185.18 & 336.2 & -32.6 & 0.07 & NRM & & & & $\mathrm{R}$ ? \\
\hline $29-4,26-28$ & 185.76 & 305.6 & 46.9 & 0.14 & 75 & 18.6 & 45.2 & 0.07 & $\mathrm{~N}$ \\
\hline $30-1,33-35$ & 186.35 & 264.1 & 8.5 & 0.06 & NRM & & & & $?$ \\
\hline $30-1,123-125$ & 187.23 & 175.1 & 37.2 & 0.13 & 25 & 11.7 & 75.7 & 0.12 & $\mathrm{~N}$ \\
\hline $30-2,30-32$ & 187.80 & 192.7 & 2.5 & 0.02 & NRM & & & & $?$ \\
\hline $30-2,118-120$ & 188.68 & 299.8 & 40.7 & 0.54 & so & 55.8 & 62.8 & 0.26 & $\mathrm{~N}$ \\
\hline $30-3,30-32$ & 189.30 & 104.0 & 19.3 & 0.02 & NRM & & & & $?$ \\
\hline $30-3,126-128$ & 190.26 & 339.2 & 43,5 & 0.11 & so & 210.8 & 69.4 & 0.11 & N? \\
\hline $30-4,22-24$ & 190.72 & 225.8 & 30.4 & 0.08 & NRM & & & & $\mathrm{N}$ ? \\
\hline $31-1,13-15$ & 191.13 & 328.4 & 58.2 & 0.21 & 50 & 281.9 & 70.7 & 0.25 & $\mathrm{~N}$ \\
\hline $31-1,129-131$ & 192.29 & 268.4 & -2.6 & 0.32 & 100 & 278.1 & -2.6 & 0.11 & $\mathrm{R}$ ? \\
\hline $31-2,13-15$ & 192.63 & 170.9 & 40.5 & 0.76 & 50 & 175.8 & 37.6 & 0.61 & $\mathrm{~N}$ \\
\hline $31-2,125-127$ & 913.75 & 250.2 & 68.7 & 0.02 & NRM & & & & $\mathrm{N}$ ? \\
\hline $31-3,117-119$ & 195.17 & 315.5 & 52.3 & 0.10 & 50 & 72.0 & 26.2 & 0.10 & $\mathrm{~N}$ ? \\
\hline $32-1,38-40$ & 196.38 & 191.4 & -30.8 & 0.12 & 25 & 139.5 & -15.5 & 0.14 & $\mathrm{R}$ \\
\hline $32-1,105-108$ & 197.03 & 256.0 & 35.6 & 0.17 & 50 & 224.8 & 51.8 & 0.03 & $\mathrm{~N}$ \\
\hline $32-2,34-36$ & 197.84 & 267.5 & 36.4 & 0.14 & 25 & 27.7 & -9.5 & 0.07 & $\mathrm{R}$ ? \\
\hline $32-2,102-104$ & 198.52 & 137.3 & 20.0 & 0.05 & NRM & & & & $\mathrm{N}$ \\
\hline $32-3,34-36$ & 199.34 & 285.3 & 14.6 & 0.36 & 50 & 289.7 & 37.5 & 0.24 & $\mathrm{~N}$ \\
\hline $32-3,104-106$ & 200.4 & 183.4 & 72.8 & 0.09 & NRM & & & & $\mathrm{N}$ ? \\
\hline $33-1 ; 48-50$ & 201.48 & 294.2 & 11.1 & 0.021 & NRM & & & & $\mathrm{N}$ ? \\
\hline $33-1,104-106$ & 202.04 & 275.7 & 45.3 & 0.07 & NRM & & & & $\mathrm{N}$ ? \\
\hline $33-2,45-47$ & 202.95 & 272.4 & 44.5 & 0.06 & NRM & & & & $\mathrm{N}$ ? \\
\hline $33-2,109-111$ & 203.59 & 293.8 & 46.0 & 0.12 & 50 & 187.8 & 53.3 & 0.20 & $\mathrm{~N}$ \\
\hline $33-3,41-43$ & 204.41 & 271.0 & 17.2 & 0.09 & NRM & & & & $\mathrm{N}$ ? \\
\hline $33-3,120-122$ & 205.20 & 82.1 & 56.3 & 0.11 & NRM & & & & $\mathrm{N}$ \\
\hline $34-2,46-48$ & 207.96 & 242.1 & -78.3 & 0.81 & 50 & 216.4 & -72.9 & 0.92 & $\mathrm{R}$ \\
\hline $34-2,106-108$ & 208.56 & 336.1 & -0.09 & NRM & & & & & $\mathrm{R}$ ? \\
\hline $35-1,120-122$ & 210.20 & 77.4 & 29.4 & 0.22 & 100 & 56.8 & 32.0 & 0.12 & $\mathrm{~N}$ \\
\hline $35-1,128-130$ & 210.3 & 31.2 & 0.11 & NRM & & & & & $\mathrm{N}$ \\
\hline
\end{tabular}

further movement. This suggests that a secondary component of normal polarity has been removed by the low alternating fields.

\section{Magnetic Polarity Record}

A polarity reversal record could not be developed for the upper part of this hole, but it could be achieved for the older sediments, and the results are summarized in Figure 6 . The position of the nannofossil and foraminiferal zones (Müller, this volume and Snyder, this volume) are also shown on this diagram, together with the anomaly numbers assigned to each reversal. Unfortunately, the section is incomplete; there are gaps in the record between the early and middle Eocene and between the Eocene and Maestrichtian. An interesting feature is the normal polarity interval corresponding to nannofossil Zone NP10, which is not obviously correlated with any specific magnetic anomaly on the standard polarity time scales of Lowrie and Alvarez (1981) or Hailwood et al. (1979).

\section{NRM Intensity and Volume Susceptibility Measurements}

Figure 7 illustrates the downhole variation in volume susceptibility and NRM intensity in the lower part of Hole 548A. The abrupt upward lithologic change that occurs at $469.9 \mathrm{~m}$ sub-bottom between nannofossil chalks and overlying brown-colored marly nannofossil chalks is correlated with a sudden increase in volume susceptibility and NRM intensity. Similarly, the distinct decrease in susceptibility and NRM intensity at $412.6 \mathrm{~m}$ sub-bottom corresponds to an abrupt lithologic change between the early Eocene marly nannofossil chalks and middle Eocene light-colored chalks. The increase in susceptibil- 


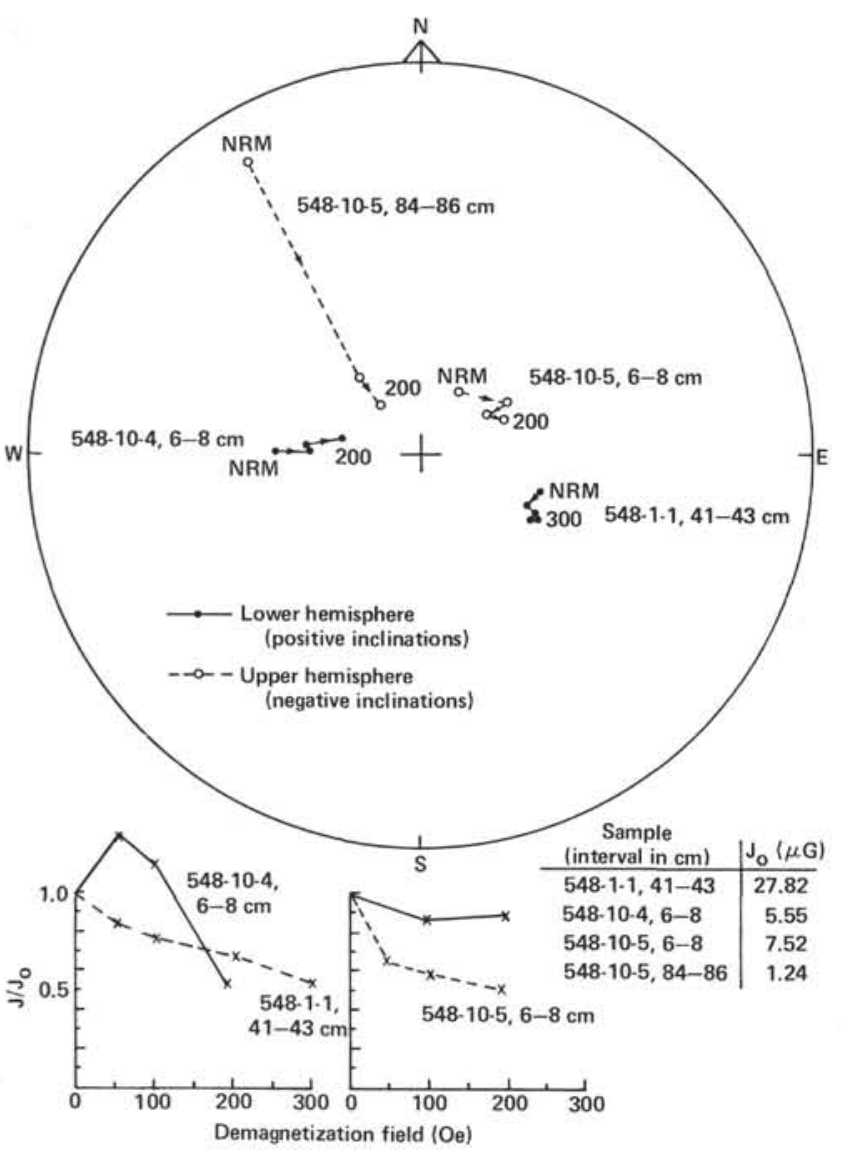

Figure 2. Behavior of four samples from Hole 548 during af demagnetization. Numbers refer to the peak applied alternating field in oersteds. The graphs illustrate the change in normalized intensity with peak demagnetization field for these samples; $\mathbf{J}=$ intensity, $\mathrm{J}_{\mathrm{o}}=$ initial intensity.

ity and NRM intensity values at $469.9 \mathrm{~m}$ sub-bottom would seem to represent an increase in the supply of terrigenous material that ceased at the end of the early Eocene. The change is of particular interest because similar results were noted at Holes $400 \mathrm{~A}$ and 401 during DSDP Leg 48 (Fig. 8). At Hole 400A this magnetic boundary occurs at a depth of $565 \mathrm{~m}$, and at Hole 401 it occurs at $173 \mathrm{~m}$. Magnetic fabric studies suggest that important changes in the direction of sediment transport might correspond to these fluctuations in lithology and magnetic intensity (Hailwood and Folami, this volume).

\section{Site 549}

At Site 549 (the second shallowest site in the Goban Spur transect), two holes were drilled near the seaward edge of the tilted block of Hercynian basement. For Hole 549A the VLHPC was used, and a sequence of Holocene to middle Eocene nannofossil and marly nannofossil oozes was recovered. Hole 549 was rotary drilled, and middle Eocene to lower Barremian chalks, mudstones, sandstones, and limestones were recovered; they rest on Hercynian basement.

\section{Paleomagnetic Results}

Approximately 400 samples were taken from Site 549; the results of the paleomagnetic measurements are listed

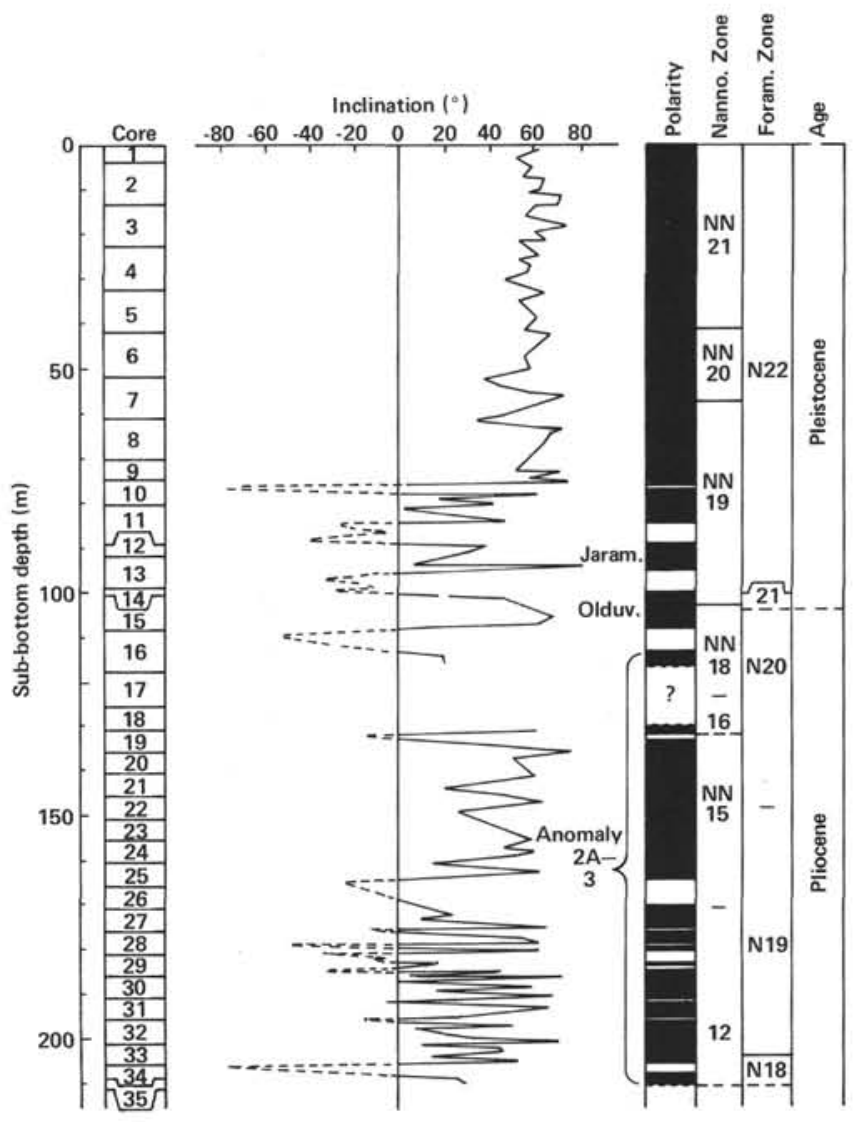

Figure 3. Downhole variation of magnetic inclination and inferred polarity reversal sequence for Hole 548. Positions of biostratigraphic zones are also shown. Normal polarity intervals shaded, reverse white.

in Table 3. At Hole 549A an abrupt change in NRM intensity occurs at the base of Core 3 . This change corresponds to the hiatus between the Pleistocene marly sediments and the underlying sequence of nannofossil oozes. Since the nannofossil oozes were very weakly magnetized, the results for this section are largely based on NRM measurements or demagnetization in very low alternating fields.

Figure 9A illustrates the behavior upon demagnetization of typical samples from Site 549. Figure 9A shows a normal polarity sample $(549 \mathrm{~A}-3-4,33-35 \mathrm{~cm})$ and a reverse polarity sample $(549 \mathrm{~A}-3-1,19-21 \mathrm{~cm})$ from Hole 549A. The latter clearly demonstrates that a secondary component is removed on demagnetization. Figure 9B shows two normal polarity samples and two reverse polarity samples from Hole 549. The results are broadly similar to those from Hole 549A. A histogram of the magnetic inclinations of all the samples from Hole 549 after af demagnetization is shown in Figure 10. The histogram is bimodal, with the two peaks representing the normal and reverse polarity samples. The abundance of normal polarity samples is due to the long section through the Cretaceous magnetic quiet zone. The broken lines on the histogram indicate the theoretical axial geocentric dipole values for this latitude. For both the normal and reverse polarity samples, the peaks are displaced away from this value toward lower inclination 


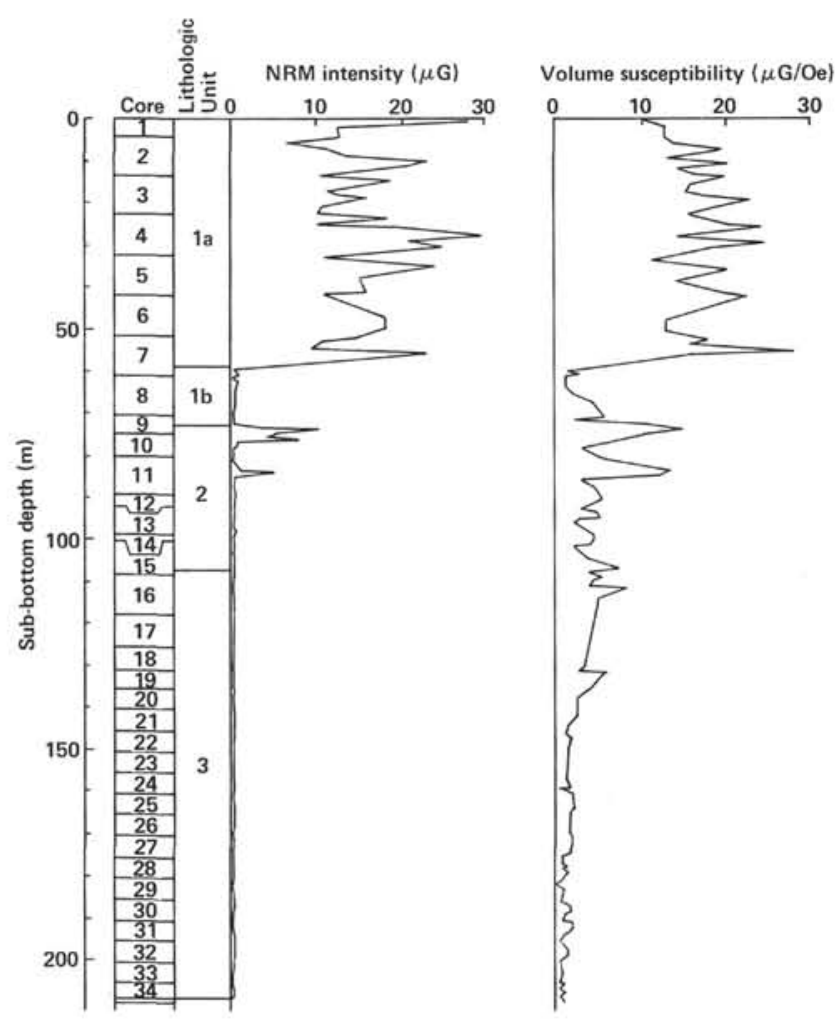

Figure 4. Correlation between volume susceptibility, NRM intensity, and lithology at Hole 548 .

values, probably as a result of the combined effects of compaction and the incomplete removal of secondary components of magnetization of normal polarity.

\section{Hole $549 A$}

The sequence of polarity reversals established at Hole 549A is shown in Figure 11. The Pleistocene sediments down to $24.00 \mathrm{~m}$ sub-bottom were of normal polarity, with the exception of one sample at $17.69 \mathrm{~m}$ sub-bottom. The results from the underlying sediments are less reliable because of the lower magnetic intensities of the nannofossil oozes. It has been impossible to establish a magnetostratigraphy for the Miocene sediments, because the section is highly condensed. The preceding section through the Oligocene and upper Eocene is more complete, and the magnetic polarity reversals deduced for this section are shown in Figure 11. It must be stressed that these results are based either on NRM measurements alone or (in the case of the slightly stronger samples) demagnetization in low alternating fields (up to 50 Oe). The biostratigraphy was used to assign magnetic anomaly numbers to the polarity reversals and to define gaps in the sequence. The results are significant, because they provide an opportunity to define the position of the Oligocene/Eocene boundary in this hole.

The position of the Oligocene/Eocene boundary in the magnetic polarity time scale is subject to some dispute. LaBrecque et al. (1977) place it in the reverse polarity interval preceding Anomaly 15, whereas Ness et al. (1980) propose that it lies at the top of Anomaly 16B.

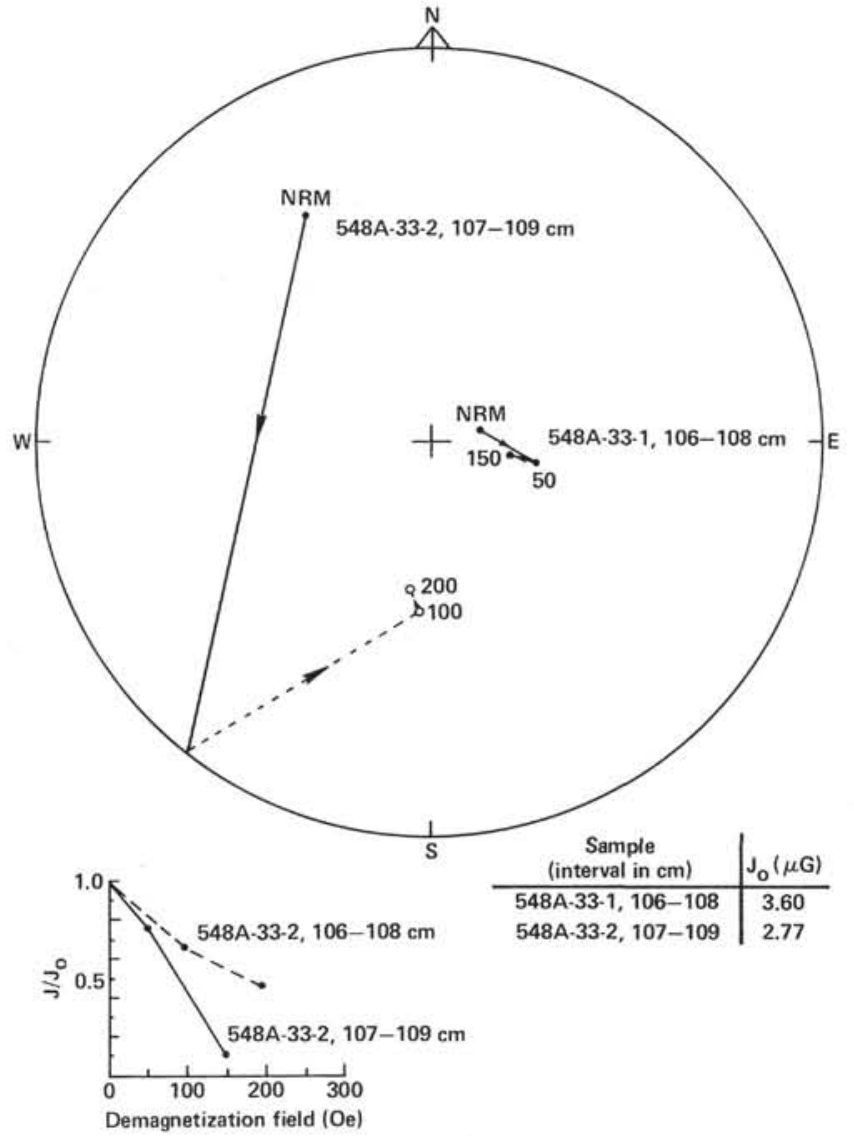

Figure 5. Demagnetization behavior of two representative samples from Hole 548A. Symbols as in Fig. 2.

In the most recent study, Lowrie and Alvarez (1981) place it in the reverse polarity interval following Anomaly 15. The work of Lowrie and Alvarez (1981) is based on combined paleomagnetic and biostratigraphic studies of relatively complete sections of pelagic limestones in the Southern Appennines and is therefore likely to provide the best direct correlation between the magneto- and biostratigraphy. On this basis the Eocene/Oligocene boundary in Hole 549A may be placed between 120.00 and $123.4 \mathrm{~m}$ sub-bottom.

\section{Hole 549}

The paleomagnetic results from the upper part of Hole 549 (down to the Paleocene/Upper Cretaceous hiatus) span a sequence of richly fossiliferous sediments of middle Eocene to late Paleocene age. This section, which is ideally suited to detailed biostratigraphic studies, proved also to be suitable for magnetostratigraphic work. The downhole variation of magnetic inclination together with the polarity reversal sequence defined is shown in Figure 12. A series of polarity reversals has been defined that is believed to correlate with marine magnetic Anomalies 18 to 26 .

It was thought at first that this section was complete, inasmuch as the biostratigraphic and magnetostratigraphic studies showed no direct evidence of breaks in 
Table 2. Paleomagnetic results, Hole 548A.

\begin{tabular}{|c|c|c|c|c|c|c|}
\hline & & & $\begin{array}{l}\text { Before } \\
\text { agnetiza }\end{array}$ & & Demagnetization & \\
\hline $\begin{array}{l}\text { Core-Section } \\
\text { (interval in cm) }\end{array}$ & $\begin{array}{l}\text { depth } \\
\text { (m) }\end{array}$ & $\begin{array}{c}\text { D } \\
\left({ }^{\circ}\right)\end{array}$ & $\begin{array}{c}1 \\
\left({ }^{\circ}\right)\end{array}$ & $\begin{array}{c}\text { Int } \\
(\mu \mathrm{G})\end{array}$ & $\begin{array}{l}\text { field } \\
(\mathrm{Oe})\end{array}$ & $\begin{array}{c}\text { D } \\
\left({ }^{\circ}\right)\end{array}$ \\
\hline $2-1,117-119$ & 216.17 & 133.0 & 29.9 & 0.035 & NRM & \\
\hline $2-2,117-119$ & 217.67 & 13.3 & 55.9 & 0.151 & 50 & 358.2 \\
\hline $2-3,27-29$ & 218.27 & 9.2 & -13.3 & 0.308 & 100 & 199.1 \\
\hline $2-3,122-134$ & 219.32 & 281.7 & 80.5 & 0.398 & NRM & \\
\hline $2-4,37-39$ & 219.57 & 51.3 & 69.5 & 0.105 & 25 & 99.0 \\
\hline $3-1,68-70$ & 225.18 & 4.0 & 37.1 & 0.059 & NRM & \\
\hline $3-1,131-133$ & 225.31 & 159.3 & -1.9 & 0.458 & 75 & 156.5 \\
\hline $3-2,15-17$ & 227.15 & 295.7 & 58.9 & 0.235 & 50 & 279.9 \\
\hline $3-3,109-111$ & 228.59 & 15.6 & 64.9 & 0.223 & 50 & 183.2 \\
\hline $3-4,27-30$ & 229.27 & 347.1 & 51.3 & 0.147 & 25 & 250.3 \\
\hline $3-4,118-121$ & 230.18 & 202.8 & 33.6 & 0.083 & NRM & \\
\hline $3-5,19-22$ & 230.69 & 105.7 & 35.7 & 0.250 & 50 & 101.3 \\
\hline $3-5,111-113$ & 231.61 & 158.8 & -15.4 & 0.038 & NRM & \\
\hline $3-6,35-36$ & 232.35 & 291.2 & 78.8 & 0.156 & NRM & \\
\hline 3-6. 104-107 & 233.4 & 117.7 & 72.0 & 0.200 & 25 & 48.0 \\
\hline $4-2,43-46$ & 234.93 & 104.0 & 87.4 & 0.181 & so & 207.2 \\
\hline $4-3,42-45$ & 236.42 & 180.9 & 71.8 & 0.107 & NRM & \\
\hline $4-4,43-46$ & 237.93 & 152.5 & 33.8 & 0.094 & NRM & \\
\hline $4-4,108-111$ & 238.58 & 22.7 & 61.0 & 0.009 & NRM & \\
\hline $4-5,6-8$ & 239.06 & 340.5 & 67.2 & 0.007 & NRM & \\
\hline $4-5,120-122$ & 240.20 & 320.0 & -11.6 & 0.106 & 40 & 331.9 \\
\hline $4-6,25-27$ & 240.75 & 166.4 & 39.5 & 0.103 & NRM & \\
\hline $4-6,124-126$ & 241.74 & 8.3 & 4.3 & 0.043 & NRM & \\
\hline $5-1,30-32$ & 242.85 & 342.8 & 55.4 & 0.067 & NRM & \\
\hline $5-1,116-118$ & 243.66 & 348.2 & 47.8 & 0.009 & NRM & \\
\hline $5-2,34-36$ & 244.34 & 110.6 & -31.5 & 0.005 & NRM & \\
\hline $5-2,92-94$ & 244.92 & 83.1 & 57.4 & 0.035 & NRM & \\
\hline $5-3,92-94$ & 246.37 & 127.8 & 48.9 & 0.005 & NRM & \\
\hline $5-4,26-28$ & 247.26 & 241.8 & 31.5 & 0.093 & NRM & \\
\hline $5-5,88-90$ & 249.38 & 42.1 & 73.7 & 0.015 & NRM & \\
\hline $5-6,131-133$ & 250.31 & 215.9 & -45.1 & 0.006 & NRM & \\
\hline $6-2,118-120$ & 254.68 & 292.9 & -45.1 & 0.006 & NRM & \\
\hline $6-3,133-135$ & 256.33 & 201.3 & -39.3 & 0.020 & NRM & \\
\hline $6-4,128-130$ & 257.78 & 9.3 & 30.2 & 0.070 & NRM & \\
\hline $7-1,137-139$ & 262.87 & 70.7 & -75.6 & 0.034 & NRM & \\
\hline $7-3,55-57$ & 265.05 & 150.3 & -31.1 & 0.010 & NRM & \\
\hline $8-1,96-98$ & 272.96 & 304.7 & 56.7 & 0.049 & NRM & \\
\hline $8-2,95-97$ & 274.45 & 350.2 & -18.6 & 0.009 & NRM & \\
\hline $8-3,55-57$ & 275.55 & 29.8 & 31.8 & 0.052 & NRM & \\
\hline $8-3,90-92$ & 275.90 & 149.9 & -27.3 & 0.010 & NRM & \\
\hline $8-1,67-69$ & 280.17 & 338.9 & 17.5 & 0.265 & NRM & \\
\hline $9-2,28-30$ & 383.25 & & 61.3 & 0.295 & 40 & 355.5 \\
\hline $9-2,106-108$ & 284.06 & 51.0 & 31.2 & 0.061 & NRM & \\
\hline $9-3,108-110$ & 285.58 & 1.7 & -27.4 & 0.117 & 25 & 56.3 \\
\hline $9-4,28-30$ & 286.28 & 29.7 & 39.1 & 0.053 & NRM & \\
\hline $9-4,120-122$ & 287.20 & 302,1 & -16.8 & 0.253 & 25 & 277.3 \\
\hline $9-5,163-165$ & 288.13 & 6.3 & 37.9 & 0.164 & 25 & 316.6 \\
\hline $9-5,120-122$ & 288.7 & 46.6 & 34.3 & 0.011 & NRM & \\
\hline $10-2,49-50$ & 292.99 & 24.5 & 51.9 & 0.295 & 25 & 10.3 \\
\hline $10-2,94-96$ & 293.44 & 333.3 & 25.4 & 0.030 & NRM & \\
\hline $10-3,34-36$ & 294.34 & 59.7 & 58.1 & 0.241 & 50 & 59.3 \\
\hline $10-3,118-120$ & 295.18 & 333.1 & 45.0 & 0.049 & NRM & \\
\hline $10-4,34-36$ & 295.84 & 266.4 & 73.6 & 0.101 & NRM & \\
\hline $10-5,36-38$ & 297.36 & 325.3 & 68.5 & 0.178 & 50 & 170.1 \\
\hline $10-6,32-34$ & 298.82 & 349.4 & 46.4 & 0.100 & NRM & \\
\hline $10-6,123-125$ & 299.73 & 62.7 & 19.4 & 0.060 & NRM & \\
\hline $11-1,101-103$ & 301.51 & 69.8 & 0.8 & 0.029 & NRM & \\
\hline $11-2,40-42$ & 302.40 & 322.2 & 72.8 & 0.062 & NRM & \\
\hline $11-2,97-99$ & 302.97 & 319.1 & 9.5 & 0.133 & NRM & \\
\hline $11-3,27-29$ & 303.79 & 300.6 & 43.6 & 0.054 & NRM & \\
\hline $11-3,108-110$ & 304.08 & 287.9 & 70.9 & 0.121 & 25 & 223.1 \\
\hline $11-4,48-50$ & 305.48 & 51.3 & -42.6 & 0.011 & NRM & \\
\hline $11-4,135-137$ & 306.35 & 356.1 & -16.8 & 0.022 & NRM & \\
\hline $11-5,19-21$ & 306.69 & 102.3 & 83.2 & 0.065 & NRM & \\
\hline $12-1,25-27$ & 310.25 & 329.2 & -10.9 & 0.036 & NRM & \\
\hline $12-2,127-129$ & 312.79 & 132.5 & -19.0 & 0.081 & NRM & \\
\hline $12-3,126-128$ & 314.26 & 200.4 & 35.7 & 0.100 & NRM & \\
\hline $12-4,116-118$ & 315.66 & 9.5 & 53.0 & 0.064 & NRM & \\
\hline $12-5,138-141$ & 317.39 & 246.5 & -21.1 & 0.047 & NRM & \\
\hline $12-6,9-11$ & 317.59 & 233.7 & -46.1 & 0.130 & NRM & \\
\hline $13-1,28-30$ & 319.80 & 117.8 & 77.7 & 0.020 & NRM & \\
\hline $13-1,136-138$ & 320.86 & 75.0 & -29.5 & 0.057 & NRM & \\
\hline $13-2,25-28$ & 321.25 & 194.7 & -25.5 & 0.021 & NRM & \\
\hline $13-2,133-136$ & 322.33 & 14.6 & -42.3 & 0.001 & NRM & \\
\hline $13-3,16-19$ & 322.66 & 46.3 & 72.6 & 0.002 & NRM & \\
\hline $13-3,114-116$ & 323.64 & 339.4 & -23.7 & 0.075 & NRM & \\
\hline $13-4,9-12$ & 324.09 & 293.6 & 23.3 & 0.063 & NRM & \\
\hline $13-4,116-119$ & 325.16 & 2.2 & -46.6 & 0.057 & NRM & \\
\hline $13-5,23-25$ & 325.73 & 195.5 & 39.8 & 0.040 & NRM & \\
\hline $13-5,144-146$ & 236.94 & 117.6 & -4.5 & 0.041 & NRM & \\
\hline $13-6,35-38$ & 327.35 & 193.7 & 13.3 & 0.762 & NRM & \\
\hline $13-6,133-136$ & 328.33 & 154.1 & 84.3 & 0.005 & NRM & \\
\hline $14-1,15-17$ & 329.15 & 337.2 & 27.6 & 0.075 & NRM & \\
\hline $14-1,123-125$ & & & & 0.001 & NRM & \\
\hline $14-2,15-17$ & 330.65 & 274.8 & -15.5 & 0.002 & NRM & \\
\hline $14-2,98-100$ & 331.48 & 321.8 & -43.6 & 0.042 & NRM & \\
\hline $14-3,37-39$ & 332.37 & 120.2 & 34.4 & 0.061 & NRM & \\
\hline $14-3,122-124$ & 333.22 & 228.2 & 37.6 & 0.002 & NRM & \\
\hline $14-4,33-35$ & 333.83 & 11.7 & -47.0 & 0.058 & NRM & \\
\hline $14-4,115-117$ & 334.15 & 279.5 & 17.9 & 0.108 & NRM & \\
\hline
\end{tabular}


Table 2. (Continued).

\begin{tabular}{|c|c|c|c|c|c|c|c|}
\hline & Sub-bottom & & $\begin{array}{l}\text { Before } \\
\text { agnetiza }\end{array}$ & & & & $\begin{array}{r}\text { A } \\
\text { demagn }\end{array}$ \\
\hline $\begin{array}{l}\text { Core-Section } \\
\text { (interval in cm) }\end{array}$ & $\begin{array}{c}\text { depth } \\
(\mathrm{m})\end{array}$ & $\begin{array}{l}\text { D } \\
\left({ }^{\circ}\right)\end{array}$ & $\begin{array}{c}1 \\
\left({ }^{\circ}\right)\end{array}$ & $\begin{array}{l}\text { Int } \\
(\mu \mathrm{G})\end{array}$ & $\begin{array}{l}\text { field } \\
(\mathrm{Oe})\end{array}$ & $\begin{array}{l}\text { D } \\
\left({ }^{\circ}\right)\end{array}$ & $\begin{array}{c}1 \\
\left({ }^{\circ}\right)\end{array}$ \\
\hline $14-5,31-33$ & 335.31 & 359.2 & 26.1 & 0.043 & NRM & & \\
\hline $14-6,81-83$ & 337.1 & 320.0 & 53.1 & 0.105 & NRM & & \\
\hline $15-1,133-135$ & 339.83 & 56.0 & -21.0 & 0.132 & 25 & 214.2 & 42.2 \\
\hline $15-2,45-47$ & 340.45 & 301.6 & 2.0 & 0.104 & NRM & & \\
\hline $15-2,108-110$ & 341.08 & 47.8 & 10.3 & 0.047 & NRM & & \\
\hline $15-3,116-118$ & 342.66 & 26.5 & -8.5 & 0.022 & NRM & & \\
\hline $15-4,48-50$ & 343.48 & 352.5 & -40.9 & 0.005 & NRM & & \\
\hline $15-5,25-27$ & 344.75 & 80.3 & 41.2 & 0.166 & 50 & 129.4 & 14.7 \\
\hline $15-6,12-14$ & 346.12 & 304.3 & 67.0 & 0.092 & NRM & & \\
\hline $16-1,28-31$ & 349.21 & 254.3 & 11.2 & 0.099 & NRM & & \\
\hline $16-2,19-21$ & 349.69 & 298.5 & 66.5 & 1.709 & 50 & 288.5 & 62.2 \\
\hline $16-3,26-29$ & 351.26 & 41.8 & 62.9 & 6.4 & 150 & 57.2 & 60.9 \\
\hline $16-4,19-21$ & 352.69 & 353.7 & -6.4 & 1.207 & 100 & 309.3 & -49.8 \\
\hline $16-4,119-121$ & 353.69 & 215.4 & 72.2 & 3.689 & 50 & 189.3 & 74.6 \\
\hline $16-5,20-22$ & 354.20 & 148.5 & 82.2 & 1.122 & 75 & 17.2 & -57.0 \\
\hline $17-1,32-34$ & 357.82 & 138.6 & 57.0 & 2.439 & 100 & 110.9 & 52.5 \\
\hline $17-1,116-118$ & 358.66 & 219.4 & 79.4 & 3.806 & 75 & 280.0 & 80.2 \\
\hline $17-2,123-125$ & 360.23 & 33.7 & 67.0 & 2.73 & NRM & & \\
\hline $17-3,34-36$ & 360.84 & 102.7 & 63.4 & 8.255 & 100 & 110.7 & 62.5 \\
\hline $17-4,106-108$ & 363.06 & 187.7 & 69.2 & 9.109 & 100 & 199.7 & 66.2 \\
\hline $17-5,114-116$ & 365.14 & 61.6 & 57.7 & 7.164 & 100 & 62.2 & 52.1 \\
\hline $17-6,130-133$ & 366.30 & 27.0 & 63.3 & 4.697 & 100 & 14.7 & 78.7 \\
\hline $18-1,25-27$ & 367.25 & 242.9 & 62.3 & 9.608 & 100 & 242.1 & 62.9 \\
\hline $18-2,101-103$ & 369.51 & 296.7 & 72.7 & 6.887 & 100 & 165.8 & 73.6 \\
\hline $18-3,32-34$ & 370.32 & 91.3 & 81.8 & 2.405 & 50 & 52.9 & 77.4 \\
\hline $19-1,25-27$ & 376.75 & 352.8 & 18.2 & 0.191 & 50 & 300.0 & 29.6 \\
\hline $19-1,144-146$ & 377.94 & 12.9 & 14.1 & 0.094 & 50 & 357.8 & 56.7 \\
\hline $19-2,16-19$ & 378.16 & 185.6 & -33.2 & 0.096 & NRM & & \\
\hline $19-3,130-132$ & 380.80 & 194.1 & 57.3 & 0.216 & 50 & 112.5 & 47.7 \\
\hline $19-4,34-37$ & 381.34 & 189.1 & 61.8 & 0.082 & NRM & & \\
\hline $19-4,108-111$ & 382.08 & 100.1 & 83.3 & 0.361 & 50 & 87.7 & 74.3 \\
\hline $19-5,18-21$ & 382.68 & 291.5 & 48.5 & 0.204 & 25 & 307.9 & 34.2 \\
\hline $19-5,105-107$ & 383.55 & 46.1 & 58.6 & 0.635 & 100 & 94.7 & 55.6 \\
\hline $19-6,35-37$ & 384.35 & 116.0 & 42.1 & 0.003 & NRM & & \\
\hline $19-6,95-97$ & 384.95 & 9.1 & -30.6 & 0.095 & NRM & & \\
\hline $20-1,108-110$ & 387.08 & 282.3 & 13.8 & 0.086 & NRM & & \\
\hline $20-2,20-23$ & 387.70 & 354.1 & -30.2 & 0.001 & NRM & & \\
\hline $20-2,109-111$ & 388.59 & 311.9 & -4.4 & 0.056 & NRM & & \\
\hline $20-3,27-30$ & 389.27 & 219.6 & 85.8 & 0.155 & NRM & & \\
\hline $20-4,11-13$ & 390.61 & 106.4 & 47.3 & 0.316 & 25 & 313.3 & -55.8 \\
\hline $21-1,23-25$ & 395.73 & 83.8 & -33.7 & 0.061 & NRM & & \\
\hline $21-1,109-111$ & & & & 0.103 & NRM & & \\
\hline $21-2,46-48$ & 397.46 & 105.1 & 23.1 & 0.181 & 25 & 70.2 & 67.4 \\
\hline $21-3,19-21$ & 398.69 & 10.3 & 20.8 & 0.124 & 25 & 21.9 & 43.6 \\
\hline $21-3,107-109$ & 399.59 & 32.4 & -8.1 & 0.121 & NRM & & \\
\hline $21-4,15-19$ & 400.13 & 338.2 & 23.6 & 0.125 & 25 & 84.4 & -29.7 \\
\hline $21-4,125-127$ & 401.25 & 28.0 & 3.1 & 0.144 & NRM & & \\
\hline $22-1,13-16$ & 405.13 & 114.8 & 67.7 & 0.408 & 25 & 147.5 & 66.3 \\
\hline $22-2,13-16$ & 406.63 & 120.7 & 77.7 & 0.436 & 25 & 165.3 & 74.8 \\
\hline $22-3,25-28$ & 408.25 & 301.7 & 57.0 & 1.729 & so & 302.7 & 48.4 \\
\hline $22-4,22-25$ & 408.72 & 199.5 & 73.2 & 3.966 & 100 & 200.8 & 62.2 \\
\hline $22-5,32-35$ & 411.32 & 194.1 & 66.5 & 6.267 & 200 & 180.0 & 45.5 \\
\hline $22-5,128-131$ & 412.28 & 8.2 & 42.1 & 1.830 & 200 & 92.9 & 40.3 \\
\hline $22-6,92-95$ & 413.42 & 40.7 & 61.5 & 12.108 & 200 & 22.1 & -21.9 \\
\hline $23-1,24-26$ & 414.74 & 6.6 & 41.5 & 33.928 & 500 & 357.4 & 8.4 \\
\hline $23-1,111-113$ & 415.63 & 306.8 & 15.7 & 28.579 & 300 & 291.3 & -0.0 \\
\hline $23-2,49-51$ & 416.49 & 66.7 & 40.3 & 44.137 & 500 & 56.3 & 9.1 \\
\hline $23-2,112-114$ & 417.12 & 239.6 & 5.8 & 12.665 & 300 & 213.0 & 4.9 \\
\hline $23-3,29-31$ & 417.79 & 287.4 & -11.9 & 29.543 & 300 & 275.5 & -34.2 \\
\hline $23-3,126-128$ & 418.76 & 320.4 & -8.8 & 17.397 & 300 & 298.3 & -29.5 \\
\hline $23-4,98-100$ & 419.98 & 239.5 & 49.8 & 11.867 & 300 & 238.9 & $-7,0$ \\
\hline $24-1,73-75$ & 425.73 & 191.6 & -5.6 & 30.121 & 400 & 192.1 & -20.4 \\
\hline $24-2,84-86$ & 427.34 & 7.8 & -23.4 & 10.636 & 500 & 357.5 & 5.8 \\
\hline $24-3,36-38$ & 428.36 & 168.7 & 42.2 & 11.362 & 300 & 202.4 & -12.8 \\
\hline $24-4,38-40$ & 429.38 & 202.4 & -12.8 & 4.857 & NRM & & \\
\hline $24-5,93-95$ & 431.93 & 111.4 & -11.0 & 10.298 & NRM & & \\
\hline $24-6,66-68$ & 433.16 & 170.0 & 16.8 & 17.228 & 400 & 185.8 & -23.2 \\
\hline $25-1,22-24$ & 433.72 & 243.2 & 38.9 & 11.391 & 400 & 234.2 & -25.9 \\
\hline $25-2,120-122$ & 436.22 & 186.1 & -23.2 & 17.006 & 400 & 190.9 & -43.4 \\
\hline $25-3,118-120$ & 477.68 & 247.6 & 71.3 & 23.004 & 400 & 257.8 & 40.8 \\
\hline $25-4,37-39$ & 438.37 & 277.0 & 33.4 & 15.797 & 300 & 274.9 & 30.1 \\
\hline $25-4,114-116$ & 439.14 & 136.4 & 19.0 & 50.411 & 500 & 123.6 & -0.1 \\
\hline $25-5,125-127$ & 440.75 & 175.5 & 55.5 & 38.795 & 500 & 274.2 & 48.9 \\
\hline $26-6,16-18$ & 450.66 & 327.4 & 42.3 & 57.201 & 400 & 332.0 & 34.2 \\
\hline $27-1,53-55$ & 453.03 & 27.4 & -20.5 & 8.871 & 200 & 28.4 & -46.6 \\
\hline $27-2,29-31$ & 454.29 & 75.9 & 61.4 & 7.225 & 200 & 93.8 & 50.3 \\
\hline $27-2,111-113$ & 455.11 & 92.1 & 55.2 & 14.727 & 600 & 110.0 & 23.8 \\
\hline $27-3,42-44$ & 455.92 & 184.5 & 54.6 & 17.335 & 300 & 188.1 & 45.5 \\
\hline $27-4,42-44$ & 457.42 & 112.5 & 53.6 & 15.712 & 600 & 94.5 & 34.5 \\
\hline $27-5,38-40$ & 458.88 & 285.7 & 72.2 & 34.249 & 400 & 292.6 & 54.6 \\
\hline $27-5,121-123$ & 459,71 & 337.8 & -24.3 & 3.125 & 200 & 213.8 & -51.6 \\
\hline $28-1,28-30$ & 463.28 & 29.0 & 40.1 & 2.485 & 150 & 0.7 & -16.3 \\
\hline $28-2,31-33$ & 463.8 & 32.3 & 72.1 & 13.973 & 200 & 293.7 & 66.6 \\
\hline $28-2,128-130$ & 464.78 & 17.6 & 42.9 & 9.631 & 200 & 24.7 & 59.0 \\
\hline $28-3,40-42$ & 465.40 & 228.5 & 68.7 & 19.249 & 700 & 317.9 & 24.0 \\
\hline $28-3,112-114$ & 466.12 & 7,4 & 59.0 & 15.569 & 200 & 5.8 & 58.8 \\
\hline $28-4,38-40$ & 466.88 & 93.8 & 65.5 & 11.77 & 300 & 254.2 & -2.5 \\
\hline $28-5,35-37$ & 468.35 & 119.2 & 67.2 & 26.017 & 300 & 289.9 & -7.7 \\
\hline $28-6,19-21$ & 469.69 & 228.6 & 79.1 & 10.611 & 100 & 230.6 & -31.6 \\
\hline
\end{tabular}


Table 2. (Continued).

\begin{tabular}{|c|c|c|c|c|c|c|c|c|c|}
\hline \multirow[b]{2}{*}{$\begin{array}{l}\text { Core-Section } \\
\text { (interval in } \mathrm{cm} \text { ) }\end{array}$} & \multirow{2}{*}{$\begin{array}{l}\text { Sub-bottom } \\
\text { depth } \\
\text { (m) }\end{array}$} & \multicolumn{3}{|c|}{$\begin{array}{c}\text { Before } \\
\text { demagnetization }\end{array}$} & \multirow{2}{*}{$\begin{array}{l}\text { Demagnetization } \\
\text { field } \\
\text { (Oe) }\end{array}$} & \multicolumn{4}{|c|}{$\begin{array}{c}\text { After } \\
\text { demagnetization }\end{array}$} \\
\hline & & $\begin{array}{l}\mathrm{D} \\
\left({ }^{\circ}\right)\end{array}$ & $\begin{array}{l}1 \\
\left({ }^{\circ}\right)\end{array}$ & $\begin{array}{c}\text { Int } \\
(\mu \mathrm{G})\end{array}$ & & $\begin{array}{l}\text { D } \\
\left({ }^{\circ}\right)\end{array}$ & $\begin{array}{l}1 \\
\left({ }^{\circ}\right)\end{array}$ & $\begin{array}{l}\text { Int } \\
(\mu \mathrm{G})\end{array}$ & Polarity \\
\hline $28-3,18-20$ & 471.18 & 19.3 & 24.6 & 3.519 & 50 & 23.4 & -16.8 & 3.898 & $\mathbf{R}$ \\
\hline $29-1,33-35$ & 471.83 & 64.3 & 50.1 & 1.449 & 150 & 82.3 & -43.9 & 3.213 & $\mathbf{R}$ \\
\hline $29-1,88-90$ & 472.38 & 8.1 & 71.1 & 1.627 & 200 & 319.6 & 11.7 & 0.249 & $\mathbf{R}$ ? \\
\hline $29-2,46-48$ & 473.46 & 92.6 & 81.1 & 6.185 & 100 & 115.9 & 79.4 & 4.085 & $\mathrm{~N}$ \\
\hline $29-2,97-99$ & 473.97 & 90.3 & 59.7 & 4.556 & 200 & 76.2 & 43.2 & 0.470 & $\mathrm{~N}$ \\
\hline $29-3,24-26$ & 474.74 & 26.5 & -26.4 & 14.104 & 100 & 37.7 & -26.9 & 14.294 & $\mathbf{R}$ \\
\hline $29-3,89-91$ & 475.39 & 42.1 & 37.6 & 0.563 & 150 & 47.5 & -63.3 & 0.387 & $\mathbf{R}$ \\
\hline $29.4,52-54$ & 476.52 & 262.7 & 73.5 & 3.812 & 100 & 266.2 & 57.5 & 3.650 & $\mathrm{~N}$ \\
\hline $29-5,53-55$ & 478.03 & 127.4 & 56.9 & 3.831 & 100 & 133.0 & 56.9 & 2.181 & $\mathrm{~N}$ \\
\hline $30-1,144-146$ & 482.44 & 18.7 & 61.7 & 1.959 & 100 & 24.1 & 60.3 & 1.722 & $\mathrm{~N}$ \\
\hline $30-2,125-127$ & 483.75 & 146.5 & 73.9 & 3.579 & 100 & 141.0 & 69.4 & 4.040 & $\mathrm{~N}$ \\
\hline $30-3,15-17$ & 484.15 & 135.3 & 70.3 & 3.104 & 100 & 136.1 & 68.9 & 2.845 & $\mathrm{~N}$ \\
\hline $31-1,27-29$ & 490.77 & 69.5 & 54.8 & 3.381 & 100 & 71.5 & 53.3 & 2.710 & $\mathrm{~N}$ \\
\hline $31-2,90-93$ & 492.90 & 350.4 & 62.7 & 3.354 & NRM & & & & $\mathrm{N}$ \\
\hline $32-1,52-54$ & 500.52 & 15.0 & 59.7 & 3.267 & 200 & 319.7 & -43.6 & 1.146 & $\mathbf{R}$ \\
\hline $32-1,113-116$ & 501.13 & 66.5 & 67.9 & 1.455 & 100 & 306.5 & -49.3 & 0.378 & $\mathbf{R}$ \\
\hline $32-2,31-34$ & 501.81 & 343.7 & 53.0 & 6.095 & 100 & 132.5 & 69.0 & 0.235 & $\mathrm{~N}$ \\
\hline $32-3,23-26$ & 503.23 & 84.7 & 81.1 & 8.042 & 100 & 139.6 & 68.2 & 1.253 & $\mathrm{~N}$ \\
\hline $32-4,43-45$ & 504.93 & 354.8 & 59.9 & 5.023 & 75 & 134.9 & 68.5 & 0.161 & $\mathrm{~N}$ \\
\hline $33-1,39-41$ & 509.89 & 314.2 & 63.5 & 1.519 & 75 & 83.2 & -14.9 & 0.502 & $\mathbf{R}$ \\
\hline $33-1,106-108$ & 510.56 & 331.1 & 34.9 & 3.604 & 200 & 185.8 & -58.7 & 1.655 & $\mathbf{R}$ \\
\hline $33-2,36-39$ & 511.36 & 47.7 & 57.2 & 3.276 & 200 & 293.4 & -56.4 & 2.207 & $\mathbf{R}$ \\
\hline $33-2,107-109$ & 512.07 & 81.2 & 79.7 & 2.775 & 150 & 101.1 & 72.8 & 0.279 & $\mathrm{~N}$ \\
\hline $33-3,120-123$ & 513.70 & 26.4 & 33.4 & 5.314 & 150 & 79.9 & -43.6 & 2.151 & $\mathbf{R}$ \\
\hline $34-1,42-45$ & 519.42 & 318.3 & 53.7 & 13.204 & 200 & 281.6 & 56.0 & 0.498 & $\mathrm{~N}$ \\
\hline $34-1,124-126$ & 520.24 & 155.5 & 79.3 & 16.318 & 100 & 169.3 & 68.0 & 2.281 & $\mathrm{~N}$ \\
\hline $34-2,135-138$ & 521.85 & 1.8 & 67.4 & 12.543 & 100 & 354.1 & 67.7 & 2.145 & $\mathrm{~N}$ \\
\hline $34-3,45-48$ & 522.45 & 36.5 & 34.6 & 4.792 & 200 & 291.7 & -50.2 & 3.002 & $\mathbf{R}$ \\
\hline $34-3,105-108$ & 523.05 & 324.8 & 60.7 & 9.752 & 100 & 82.2 & 28.0 & 0.260 & $?$ \\
\hline $34-4,115-118$ & 524.65 & 146.7 & 77.2 & 10.008 & 100 & 162.9 & 60.4 & 1.510 & $\mathrm{~N}$ \\
\hline $34-5,116-119$ & 526.16 & 115.4 & 51.2 & 9.104 & 50 & 131.5 & 50.3 & 4.118 & $\mathrm{~N}$ \\
\hline $34-6,112-114$ & 527.62 & 290.8 & 66.9 & 8.674 & 50 & 279.8 & 67.0 & 4.202 & $\mathrm{~N}$ \\
\hline $34-7,11-14$ & 528.11 & 296.0 & -71.7 & 8.139 & 50 & 261.6 & -74.0 & 4.576 & $\mathbf{R}$ \\
\hline $35-1,27-29$ & 528.77 & 343.0 & 61.4 & 14.277 & 50 & 335.1 & 62.1 & 8.728 & $\mathrm{~N}$ \\
\hline
\end{tabular}

Note: Symbols and abbreviations as in Table 1.

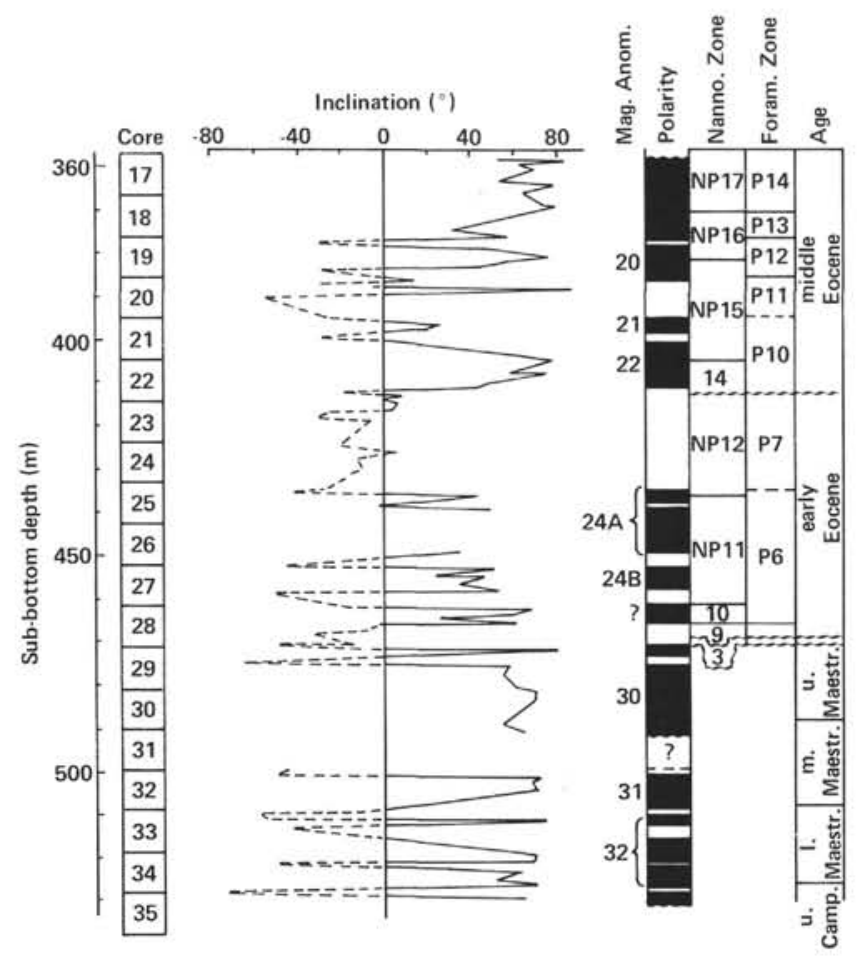

Figure 6. Downhole plot of magnetic inclination after optimum demagnetization and inferred polarity reversal sequence for Hole 548A.

sedimentation. However, at this site nannoplankton Zone NP10 is unusually short compared with the overlying zones. Furthermore, more than 45 thin bentonite layers have been recognized at this level at Site 550 , but they do not appear to be present at Site 549 (Knox, this vol- ume). This suggests that the upper part of nannoplankton Zone NP10 is probably absent at Site 549.

The sequence below the Paleocene/Maestrichtian hiatus is less complete. Figure 13 shows the paleomagnetic results for the cores that were sampled. The two normal polarity intervals in the Maestrichtian and Campanian are thought to represent Anomalies 32 and 33, respectively. They are preceded by a long normal polarity interval that extends from the top of the Santonian to the lower Barremian. In this long Cretaceous normal polarity interval three short polarity reversals (each one represented by only a single sample) were detected in Cores 37,48 , and 83 , respectively. The upper two are of midAlbian age, and the lower one is Barremian. Anomaly MO was not identified in this section but probably corresponds to the hiatus between early Albian and Barremian sediments. The paleomagnetic results from this hole indicate that the base of the Cretaceous quiet zone was not reached at Site 549. Thus, according to the van Hinte (1976) polarity time scale, which places the base of the Cretaceous normal polarity interval close to the base of the Barremian, the sediments lying directly above basement at Site 549 are not older than Barremian in age.

\section{NRM Intensity and Volume Susceptibility}

Figure 14 shows graphs of the downhole variation of NRM intensity and volume susceptibility. The general increase in NRM intensity and volume susceptibility below Core 8 corresponds to the lithologic change between light-colored nannofossil chalks and brown and gray nannofossil chalks. This increase in both properties in nannoplankton Zone NP14 agrees with the results from Site 548 and Hole $400 \mathrm{~A}$ of Leg 48. 

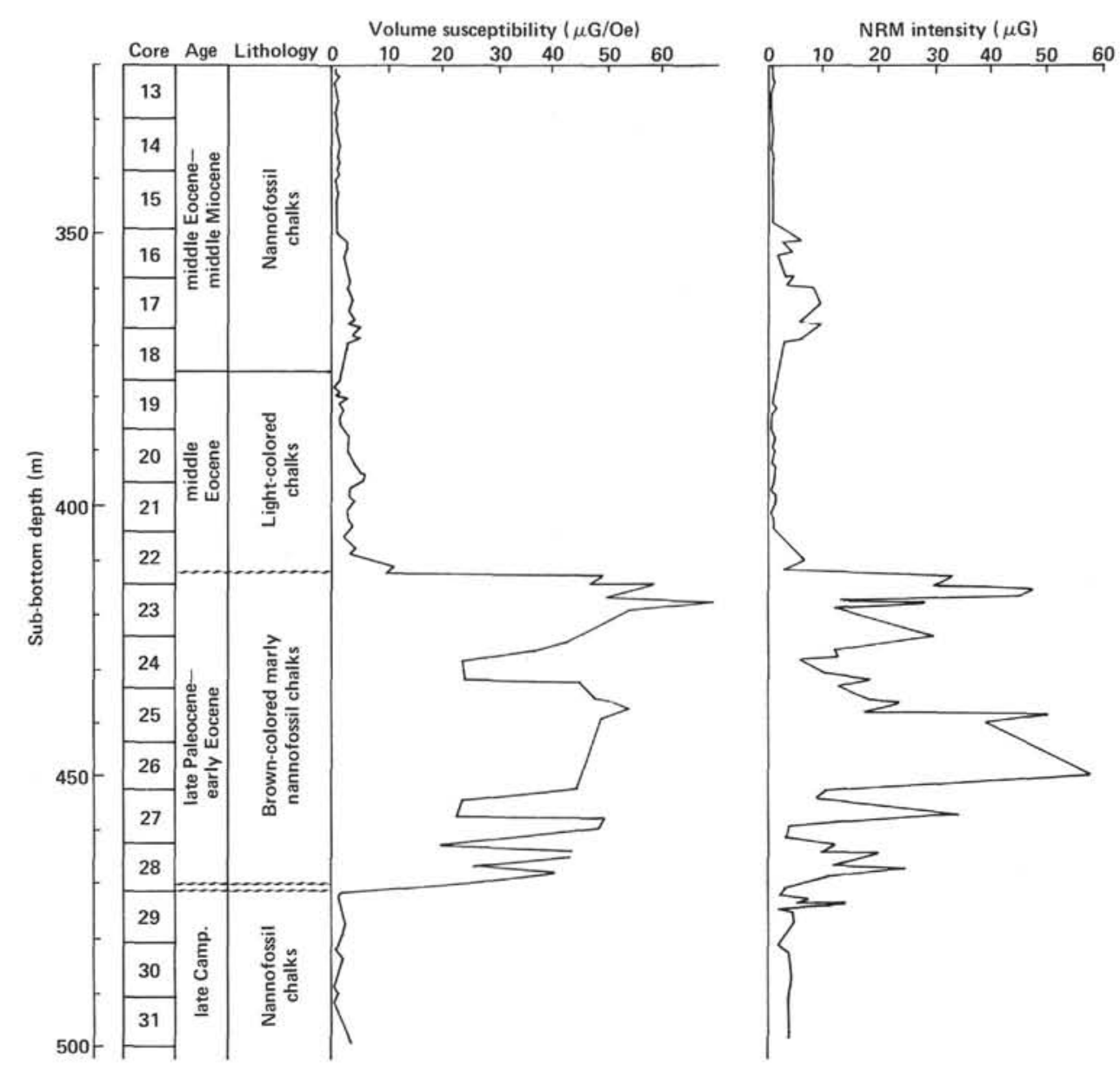

Figure 7. Correlation between volume susceptibility, NRM intensity, and lithology at Hole 548A. Interval between unconformities in Core 28 is early Paleocene in age.

\section{Site 550}

At Site 550 two holes (550 and 550B) were rotary drilled on the Porcupine Abyssal Plain. This site was at the greatest water depth of the sites drilled in the transect across the Goban Spur. Hole 550 was first washed to $99.5 \mathrm{~m}$ sub-bottom and then rotary drilled to a depth of $527 \mathrm{~m}$. Hole $550 \mathrm{~B}$ was washed to $456 \mathrm{~m}$ sub-bottom and then continuously cored to $720.5 \mathrm{~m}$ sub-bottom. The overlap between the two holes drilled at Site 550 resulted in the acquisition of a complete section across the Cretaceous/Tertiary boundary. The sediments recovered at this site were a sequence of Pliocene to late Albian nannofossil chalks and mudstones resting on oceanic basement. Hiatuses occur in the Miocene and early Oligocene to middle Eocene.

\section{Paleomagnetic Measurements}

Approximately 350 samples were measured from Site 550 , and the paleomagnetic results for these samples are summarized in Table 4 . The demagnetization behavior of representative normal and reverse polarity samples is shown in Figure 15. As with the samples from the previous two sites, the reverse polarity samples possess a low-coercivity component of normal polarity that is removed by demagnetization in low alternating fields. The normal polarity samples maintain a well-defined direction with a positive inclination during af demagnetization in fields up to 200 Oe.

\section{Polarity Reversal Stratigraphy}

Graphs of the variation of magnetic inclination with depth for Holes 550 and 550B are shown in Figures 16 and 17 , respectively. At Hole 550 a series of short normal polarity zones was identified in Cores 5 to 10 ; the zones span nannoplankton Zones NN15 to NN12 and foraminifer Zones N20 to N19 and are believed to represent the sequence of short normal polarity intervals that characterize Anomalies 2A and 3.

The recovery of the underlying Miocene deposits was relatively poor, and because of the high frequency of geomagnetic field reversals during the Miocene an unambiguous interpretation of the sequence of polarity reversals observed is not possible.

Three hiatuses have been identified from biostratigraphic evidence in the upper half of Core 24. The hiatuses represent a span of approximately 25 m.y., an interval that extends through the early Eocene into the Oligocene. The sediments below Core 24 had notably higher NRM intensities and volume susceptibilities, and recovery improved considerably, enabling the sequence of polarity reversals to be identified. These reversals 

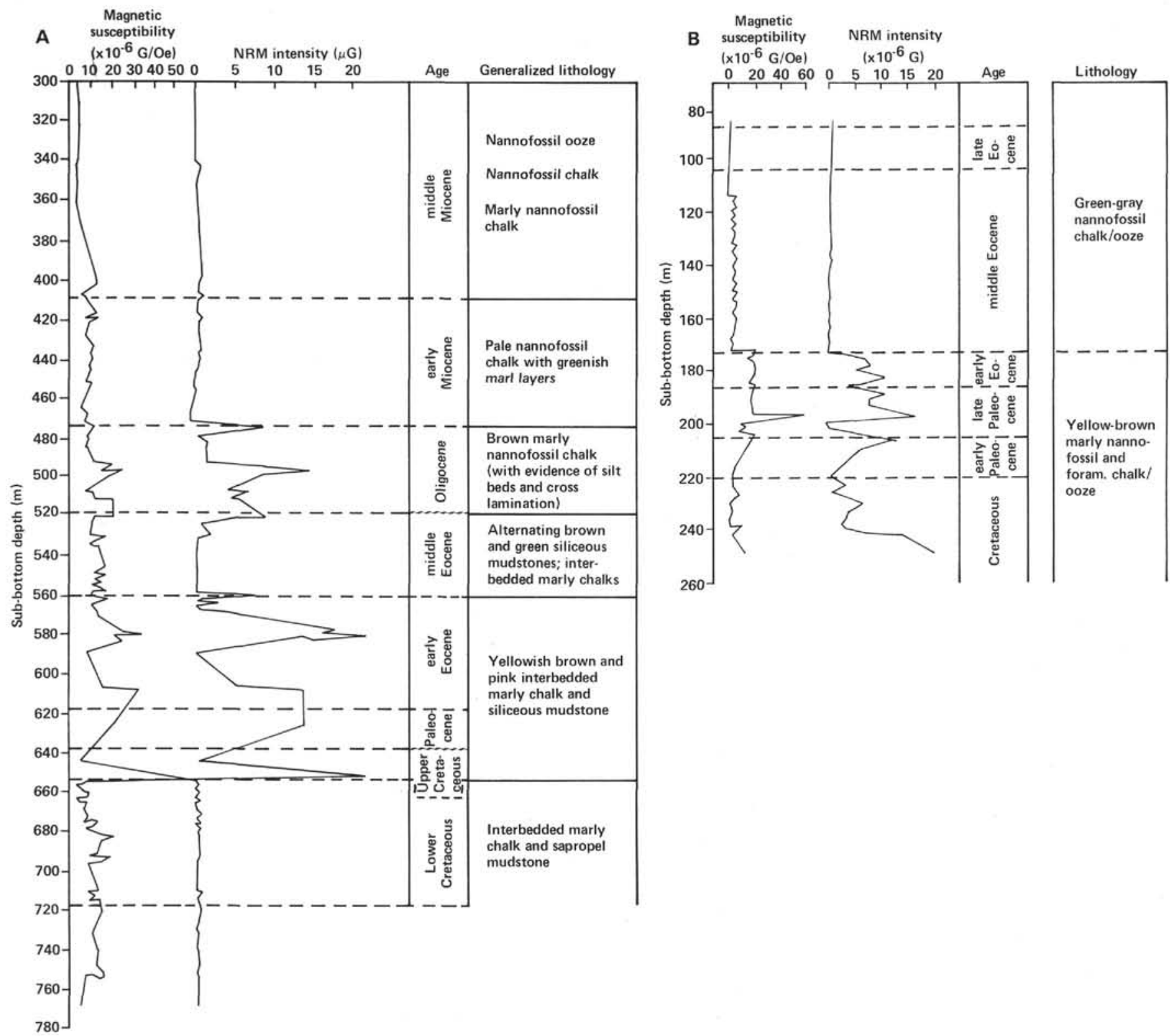

Figure 8. Leg 48 correlation between magnetic susceptibility, NRM intensity, and lithology. A. Hole 400A. B. Hole 401. (From Hailwood, 1979.)

span the Paleocene and early Eocene and are thought to represent Anomalies 22 to 27, although the section is incomplete because of a late Paleocene hiatus.

The identification of Anomalies 28, 29, and 30 was not straightforward in Hole 550, but fortunately the uppermost part of Hole 550B overlapped the lower part of Hole 550 so that the section from the Maestrichtian to early Paleocene was repeated. This permitted unambiguous identification of these anomalies in both holes. The position of Anomaly 29 is of particular interest, inasmuch as the Cretaceous/Tertiary boundary is generally accepted as lying within the reverse polarity interval immediately preceding this anomaly (Lowrie and $\mathrm{Al}$ varez, 1981 and Hailwood et al., 1979). Detailed paleomagnetic sampling has allowed the base of Anomaly 29 to be defined within $\pm 2 \mathrm{~cm}$ at Hole 550B (between 469.67 and $469.71 \mathrm{~m}$ sub-bottom). This supports the micropaleontological interpretation that places the Cretaceous/Tertiary boundary in Hole 550B within Core 2, Section 3.

In Core 8 at Hole 550B a change in polarity was recorded in lithologic Subunit 3b (at $526.8 \mathrm{~m}$ sub-bottom) within sediments that were initially interpreted as a single thick turbidite unit. Further sampling confirmed the change in polarity and enabled its position to be precisely located (at $526.80 \pm 0.02 \mathrm{~m}$ sub-bottom). The presence of a polarity reversal in this unit is inconsistent with the instantaneous emplacement of these massive light-colored beds. A more detailed examination of the core revealed the presence of fine banding in the vicinity of the polarity change that could represent a boundary between two separate turbiditic layers. This well-defined polarity change in Core 8 suggests the presence of a short reverse polarity interval within Anomaly 31 . 
Table 3. Paleomagnetic results, Site 549.

\begin{tabular}{|c|c|c|c|c|c|c|c|}
\hline & Sub-bottom & & $\begin{array}{r}\text { Before } \\
\text { nagnetiz }\end{array}$ & & Demagnetization & & demag \\
\hline $\begin{array}{l}\text { Core-Sample } \\
\text { (interval in } \mathrm{cm} \text { ) }\end{array}$ & $\begin{array}{l}\text { depth } \\
\text { (m) }\end{array}$ & $\begin{array}{l}\mathrm{D} \\
\left({ }^{\circ}\right)\end{array}$ & $\begin{array}{c}\mathrm{I} \\
\left({ }^{\circ}\right)\end{array}$ & $\begin{array}{l}\text { Int } \\
(\mu \mathrm{G})\end{array}$ & $\begin{array}{l}\text { field } \\
\text { (Oe) }\end{array}$ & $\begin{array}{l}\mathrm{D} \\
\left({ }^{\circ}\right)\end{array}$ & $\begin{array}{l}1 \\
\left({ }^{\circ}\right)\end{array}$ \\
\hline Hole $549 \mathrm{~A}$ & & & & & & & \\
\hline $1-1,18-20$ & 0.18 & 99.5 & 71.4 & 5.568 & so & 114.8 & 73.1 \\
\hline $1-2,52-55$ & 2.02 & 113.1 & 76.9 & 11.494 & so & 119.1 & 72.1 \\
\hline $1-3,47-50$ & 3.47 & 95.8 & 71.0 & 10.622 & so & 103.1 & 63.4 \\
\hline $1-4,61-63$ & 5.11 & 270.6 & 57.7 & 13.832 & so & 266.8 & 60.6 \\
\hline $1-5,26-29$ & 6.29 & 98.7 & 71.0 & 12.785 & 50 & 103.8 & 69.5 \\
\hline $2-1,124-126$ & 9.24 & 215.4 & 63.2 & 11.105 & 50 & 197.8 & 62.1 \\
\hline $2-2,124-126$ & 10.74 & 309.7 & 37.3 & 16.102 & 50 & 299.7 & 41.0 \\
\hline $2-3,114-117$ & 12.14 & 331.3 & 59.0 & 9.563 & 50 & 329.3 & 59.5 \\
\hline $2-4,35-38$ & 12.85 & 229.8 & 46.0 & 9.497 & 50 & 227.1 & 43.9 \\
\hline $2-5,36-38$ & 14.36 & 241.6 & 61.8 & 19.812 & so & 247.0 & 65.6 \\
\hline $2-6,38-40$ & 15.88 & 238.0 & 52.5 & 26.364 & 50 & 235.7 & 54.7 \\
\hline $3-1,19-21$ & 17.69 & 231.3 & 5.1 & 75.908 & 150 & 314.6 & -21.1 \\
\hline $3-1,115-117$ & 19.64 & 321.9 & 72.6 & 10.211 & 200 & 350.4 & 80.6 \\
\hline $3-2,28-30$ & 19.28 & 333.4 & 65.8 & 14.047 & 100 & 341.7 & 59.9 \\
\hline $3-3,52-54$ & 21.02 & 158.7 & 77.3 & 5.674 & 50 & 181.9 & 69.4 \\
\hline $3-4,33-35$ & 22.33 & 136.0 & 68.6 & 4.970 & 50 & 136.9 & 66.5 \\
\hline $3-5,50-52$ & 24.00 & 67.2 & 70.5 & 21.084 & 50 & 68.0 & 69.5 \\
\hline $3-5,129-131$ & 24.79 & 64.5 & -25.7 & 1.429 & 150 & 297.4 & -67.3 \\
\hline $3-6,69-71$ & 25.69 & 253.4 & -59.6 & 2.583 & 50 & 264.2 & -73.4 \\
\hline $4-1,38-40$ & 27.38 & 325.9 & -48.2 & 0.155 & 25 & 237.4 & -27.2 \\
\hline $4-2,38-40$ & 28.88 & 101.9 & -16.0 & 0.009 & NRM & & \\
\hline $4-2,106-108$ & 29.56 & 276.6 & 29.9 & 10.039 & NRM & & \\
\hline $4-2,38-40$ & 30.38 & 112.3 & 2.6 & 0.027 & NRM & & \\
\hline $4-3,136-138$ & 31.36 & 271.5 & 64.0 & 0.035 & NRM & & \\
\hline $4-4,38-40$ & 31.88 & 39.5 & -16.6 & 0.012 & NRM & & \\
\hline $4-4,119-121$ & 32.69 & 264.4 & 51.3 & 0.050 & NRM & & \\
\hline $4-5,36-38$ & 33.36 & 144.8 & 29.7 & 0.039 & NRM & & \\
\hline $4-5,117-119$ & 34.17 & 359.0 & 38.5 & 0.022 & NRM & & \\
\hline $4-6,29-31$ & 34.79 & 258.1 & -9.8 & 24.208 & 50 & 234.6 & -9.6 \\
\hline $4-6,116-118$ & 35.56 & 208.7 & -72.2 & 0.193 & 50 & 216.9 & -55.6 \\
\hline $5-1,60-62$ & 37.10 & 09.7 & 33.0 & 0.045 & NRM & & \\
\hline $5-2,62-64$ & 38.62 & 95.6 & 13.4 & 0.031 & NRM & & \\
\hline $5-3,45-47$ & 39.95 & 202.3 & 49.4 & 3.035 & 50 & 263.4 & 54.5 \\
\hline $5-4,40-42$ & 41.40 & 152.6 & 44.1 & 0.056 & NRM & & \\
\hline $5-4,107-109$ & 42.07 & 202.3 & 44.1 & 0.064 & NRM & & \\
\hline $5-5,46-48$ & 42.96 & 71.7 & -19.0 & 0.017 & NRM & & \\
\hline $5-5,91-93$ & 43.41 & 295.5 & 33.0 & 0.084 & NRM & & \\
\hline $5-6,45-47$ & 47.45 & 339.3 & 69.9 & 0.065 & NRM & & \\
\hline $5-6,84-86$ & 44.84 & 306.8 & 54.2 & 0.082 & NRM & & \\
\hline $6-1,120-122$ & 47.20 & 268.7 & 62.1 & 0.176 & 25 & 130.7 & -56.4 \\
\hline $6-2,40-42$ & 47.90 & 249.1 & -36.7 & 0.041 & NRM & & \\
\hline $6-2,122-124$ & 48.72 & 312.4 & 42.3 & 0.022 & NRM & & \\
\hline $6-3,68-70$ & 49.68 & 283.5 & 14.4 & 0.034 & NRM & & \\
\hline $6-3,132-134$ & 50.32 & 308.0 & -31.6 & 0.043 & NRM & & \\
\hline $6-4,54-56$ & 51.04 & 66.4 & -70.1 & 0.064 & NRM & & \\
\hline $6-4,105-107$ & 51.55 & 83.4 & -59.8 & 0.595 & 50 & 183.3 & 13.7 \\
\hline $6-5,59-61$ & 52.59 & 172.1 & 26.9 & 0.034 & NRM & & \\
\hline $6-5,120-122$ & 53.20 & 325.0 & -20.2 & 0.025 & NRM & & \\
\hline $6-6,48-50$ & 53.48 & 113.9 & -51.5 & 0.036 & NRM & & \\
\hline $6-6,99-101$ & 54.49 & 146.2 & -43.2 & 0.033 & NRM & & \\
\hline $7-1,47-49$ & 55.97 & 349.9 & -0.3 & 1.197 & 200 & 348.3 & 10.3 \\
\hline $7-1,103-105$ & 56.53 & 309.1 & 54.5 & 0.550 & 75 & 297.0 & 40.2 \\
\hline $7-2,48-50$ & 57.48 & 313.0 & 42.3 & 2.180 & 50 & 319.1 & 46.0 \\
\hline $7-3,101-103$ & 59.51 & 324.1 & 55.0 & 2.762 & 50 & 327.8 & 55.7 \\
\hline $7-4,55-57$ & 60.55 & 342.1 & 24.2 & 1.072 & 50 & 331.4 & 32.5 \\
\hline $7-5,58-60$ & 62.08 & 346.4 & 45.3 & 3.189 & 50 & 349.3 & 52.7 \\
\hline $7-5,101-103$ & 62.51 & 30.5 & 31.9 & 1.747 & 150 & 48.6 & 37.2 \\
\hline $7-6,55-57$ & 63.55 & 69.1 & 42.3 & 0.260 & so & 71.0 & 52.1 \\
\hline $7-6,98-100$ & 63.98 & 259.2 & 80.0 & 0.260 & 50 & 209.0 & -25.9 \\
\hline $8-1,33-35$ & 65.33 & 90.6 & -4.6 & 0.088 & NRM & & \\
\hline $8-2,33-35$ & 66.83 & 358.0 & 1.1 & 0.001 & NRM & & \\
\hline $8-2,122-124$ & 67.72 & 248.0 & -71.0 & 0.069 & NRM & & \\
\hline $8-3,33-35$ & 68.33 & 278.7 & 47.8 & 0.002 & NRM & & \\
\hline $8-4,33-35$ & 69.83 & 312.7 & 10.7 & 0.013 & NRM & & \\
\hline $8-5,33-35$ & 71.33 & 108.6 & 24.6 & 0.038 & NRM & & \\
\hline $8-6,33-35$ & 72.83 & 346.7 & 56.7 & 0.035 & NRM & & \\
\hline $9-1,23-25$ & 74.73 & 174.3 & -42.1 & 0.412 & so & 167.4 & -31.2 \\
\hline $9-1,120-122$ & 75.70 & 247.4 & 45.0 & 0.241 & so & 279.2 & 49.4 \\
\hline $9-2,23-25$ & 76.23 & 314.2 & 25.9 & 0.003 & NRM & & \\
\hline $9-2,120-122$ & 77.20 & 210.9 & 55.6 & 0.124 & NRM & & \\
\hline $9-3,23-25$ & 77.73 & 117.6 & -5.4 & 0.000 & NRM & & \\
\hline $9-3,120-122$ & 78.70 & 206.9 & 27.7 & 0.095 & NRM & & \\
\hline $9-4,23-25$ & 79.23 & 244.7 & 33.2 & 0.043 & NRM & & \\
\hline $9-4,120-122$ & 80.20 & 112.8 & 51.1 & 37.06 & 150 & 116.0 & 27.0 \\
\hline $9-5,23-25$ & 80.73 & 186.9 & 2.4 & 0.090 & NRM & & \\
\hline $9-5,120-122$ & 81.70 & 7.7 & -67.3 & 0.100 & NRM & & \\
\hline $9-6,23-25$ & 82.23 & 74.5 & -30.4 & 0.015 & NRM & & \\
\hline $10-1,46-48$ & 84.46 & 144.9 & -49.9 & 0.021 & NRM & & \\
\hline $10-1,125-127$ & 85.25 & 291.9 & -5.8 & 0.027 & NRM & & \\
\hline $10-2,46-48$ & 85.96 & 165.2 & 24.0 & 0.035 & NRM & & \\
\hline $10-2,125-127$ & 86.75 & 215.4 & -10.0 & 0.036 & NRM & & \\
\hline $10-3,46-48$ & 87.46 & 30.3 & -25.1 & 0.024 & NRM & & \\
\hline $10-3,125-127$ & 88.25 & 306.5 & 8.5 & 0.027 & NRM & & \\
\hline $10-4,46-48$ & 88.96 & 188.3 & 72.2 & 0.068 & NRM & & \\
\hline $10-4,125-127$ & 89.75 & 100.3 & 42.7 & 0.063 & NRM & & \\
\hline $10-5,46-48$ & 90.46 & 358.1 & 54.2 & 0.036 & NRM & & \\
\hline $10-5,115-117$ & 91.15 & 241.6 & -39.7 & 0.117 & NRM & & \\
\hline $10-6,48-50$ & 91.98 & 337.8 & -43.7 & 1.758 & so & 355.9 & -30.7 \\
\hline
\end{tabular}


Table 3. (Continued).

\begin{tabular}{|c|c|c|c|c|c|c|c|}
\hline & & & $\begin{array}{r}\text { Before } \\
\text { nagnetize }\end{array}$ & & & & demag \\
\hline $\begin{array}{l}\text { Core-Sample } \\
\text { (interval in cm) }\end{array}$ & $\begin{array}{l}\text { depth } \\
\text { (m) }\end{array}$ & $\begin{array}{c}\text { D } \\
\left({ }^{\circ}\right)\end{array}$ & $\begin{array}{l}1 \\
\left({ }^{\circ}\right)\end{array}$ & $\begin{array}{l}\text { Int } \\
(\mu \mathrm{G})\end{array}$ & $\begin{array}{l}\text { field } \\
(\mathrm{Oe})\end{array}$ & $\begin{array}{l}\mathrm{D} \\
\left({ }^{\circ}\right)\end{array}$ & $\begin{array}{l}1 \\
\left({ }^{\circ}\right)\end{array}$ \\
\hline Hole 549A (Cont.) & & & & & & & \\
\hline $10-6,125-127$ & 92.75 & 43.8 & -52.8 & 0.206 & 50 & 344.5 & -58.2 \\
\hline $11-1,58-61$ & 94.08 & 253.7 & 21.9 & 0.045 & NRM & & \\
\hline $11-2,22-24$ & 95.22 & 66.8 & 19.8 & 0.016 & NRM & & \\
\hline $11-2,112-115$ & 96.12 & 260.5 & -23.7 & 0.017 & NRM & & \\
\hline $11-3,49-52$ & 97.0 & 230.2 & -48.5 & 0.009 & NRM & & \\
\hline $11-4,52-54$ & 98.52 & 262.4 & -31.4 & 0.006 & NRM & & \\
\hline $11-5,51-54$ & 100.01 & 184.9 & -23.3 & 0.022 & NRM & & \\
\hline $11-5,118-120$ & 100.68 & 193.9 & -38.6 & 0.076 & NRM & & \\
\hline $11-6,54-57$ & 101.54 & 41.6 & 14.8 & 0.021 & NRM & & \\
\hline $11-6,122-125$ & 102.22 & 279.3 & 1.2 & 0.021 & NRM & & \\
\hline $12-1,36-39$ & 103.36 & 270.4 & 8.8 & 0.176 & 25 & 268.2 & -11.4 \\
\hline $12-1,115-118$ & 104.15 & 191.3 & -72.0 & 0.016 & NRM & & \\
\hline $12-2,48-51$ & 104.98 & 178.2 & 1.2 & 0.071 & NRM & & \\
\hline $13-1,41-44$ & 106.41 & i7 7.4 & 25.7 & 0.044 & NRM & & \\
\hline $13-2,43-46$ & 107.93 & $i 70 \mathrm{i}$ & -56.8 & 0.091 & NRM & & \\
\hline $13-3,41-44$ & 109.41 & $12 s .1$ & -75.9 & 0.012 & NRM & & \\
\hline $14-1,44-47$ & 111.44 & 82.7 & -2.2 & 0.277 & 25 & 76.3 & 31.2 \\
\hline $14-1,109-111$ & 112.09 & 84.5 & 38.6 & 0.011 & NRM & & \\
\hline $14-2,35-38$ & 112.85 & 170.1 & 36.3 & 0.405 & 25 & 133.6 & 48.2 \\
\hline $15-1,35-38$ & 116.35 & 11.0 & 1.7 & 0.331 & 25 & 226.9 & 48.3 \\
\hline $15-2,24-27$ & 117,74 & 98.1 & -55.8 & 0.061 & NRM & & \\
\hline $15, \mathrm{CC}(24-27)$ & 119.24 & 340.0 & 80.3 & 0.120 & NRM & & \\
\hline $16-1,56-59$ & 120.06 & 30.7 & -41.1 & 0.197 & 25 & 66.4 & -39.7 \\
\hline $16-1,110-113$ & 120.60 & 335.7 & -9.6 & 0.099 & NRM & & \\
\hline $17-1,44-47$ & 122.94 & 14.8 & -24.0 & 1.242 & 25 & 13.8 & -24.6 \\
\hline $17-1,91-93$ & 123.41 & 37.8 & 53.2 & 0.044 & NRM & & \\
\hline $17-2,26-29$ & 124.26 & 157.0 & 11.4 & 0.099 & NRM & & \\
\hline $18-1,84-87$ & 126.34 & 154.1 & 66.3 & 0.440 & 25 & 248.2 & 63.5 \\
\hline $21-1,47-49$ & 129.47 & 111.9 & -81.3 & 0.329 & 25 & 53.9 & 62.0 \\
\hline $24-1,46-48$ & 132.46 & 206.3 & -36.0 & 0.080 & NRM & & \\
\hline $24-1,86-88$ & 132.86 & 96.4 & -29.8 & 0.089 & NRM & & \\
\hline $24-2,28-30$ & 133.78 & 237.5 & 72.8 & 0.152 & NRM & & \\
\hline $25-1,35-38$ & 135.35 & 165.4 & -36.6 & 0.248 & 25 & 83.4 & -23.8 \\
\hline $25-2,26-28$ & 136.76 & 119.4 & 26.6 & 0.298 & 50 & 22.1 & 41.0 \\
\hline $25-2,97-99$ & 137.47 & 166.9 & -32.9 & 0.058 & NRM & & \\
\hline $26-1,54-55$ & 138.03 & 224.6 & 37.6 & 0.029 & NRM & & \\
\hline $27-1,52-54$ & 139.02 & 268.3 & 61.4 & 0.184 & 25 & 204.9 & 20.2 \\
\hline $27-1,112-114$ & 139.62 & 197.8 & 59.5 & 0.067 & NRM & & \\
\hline $28-1,27-30$ & 140.27 & 66.0 & -8.6 & 0.118 & NRM & & \\
\hline $28-2,30-32$ & 141.80 & 162.9 & 14.7 & 0.165 & 25 & 246.0 & 74.8 \\
\hline $32-1,44-46$ & 147.94 & 168.5 & 28.0 & 0.208 & 25 & 174.3 & -8.3 \\
\hline $32-1,114-116$ & 148.64 & 136.4 & -20.2 & 0.149 & NRM & & \\
\hline $32-2,19-21$ & 149.19 & 140.3 & -2.8 & 0.246 & 25 & 170.1 & -23.3 \\
\hline $32-2,84-86$ & 149.84 & 185.4 & -55.8 & 0.038 & NRM & & \\
\hline $33-1,42-44$ & 150.92 & 91.1 & 75.8 & 0.153 & 25 & 58.6 & 57.8 \\
\hline $33-1,114-116$ & 151.64 & 43.2 & 44.9 & 0.067 & NRM & & \\
\hline $33-2,42-44$ & 152.42 & 80.4 & 42.9 & 0.173 & 25 & 69.8 & -24.4 \\
\hline $33-2,114-116$ & 153.14 & 185.4 & -55.8 & 0.038 & NRM & & \\
\hline $34-1,38-40$ & 155.88 & 217.1 & -33.9 & 0.121 & NRM & & \\
\hline $34-1,120-123$ & 156.20 & 316.8 & -44.5 & 0.126 & NRM & & \\
\hline $35-1,38-40$ & 160.88 & 166.8 & 78.8 & 0.089 & NRM & & \\
\hline $36, \mathrm{CC}(12-14)$ & 170.12 & 31.6 & 071.7 & 0.037 & NRM & & \\
\hline $37, \mathrm{CC}(20-22)$ & 175.20 & 345.1 & -40.1 & 39.546 & 50 & 343.8 & -41.0 \\
\hline $38-1,23-25$ & 175.73 & 100.4 & -17.9 & 0.029 & NRM & & \\
\hline $39-1,38-40$ & 180.88 & 87.4 & -24.6 & 0.069 & NRM & & \\
\hline $39-1,120-123$ & 181.70 & 356.1 & 42.1 & 0.102 & NRM & & \\
\hline $39-2,38-40$ & 182.38 & 0.7 & -38.9 & 0.062 & NRM & & \\
\hline $40-1,25-27$ & 185.75 & 338.1 & -19.7 & 0.014 & NRM & & \\
\hline $40-1,104-106$ & 186.54 & 280.0 & 21.6 & 0.091 & NRM & & \\
\hline Hole 549 & & & & & & & \\
\hline $2-1,113-116$ & 199.63 & 174.0 & 5.5 & 0.023 & NRM & & \\
\hline $2-1,121-123$ & 199.71 & 358.1 & 2.4 & 0.060 & NRM & & \\
\hline $2-1,132-134$ & 199.82 & 344.5 & 67.1 & 0.019 & NRM & & \\
\hline $2-2,31-35$ & 200.31 & 286.4 & -35.3 & 0.019 & NRM & & \\
\hline $2-2,125-127$ & 201.25 & 201.8 & 59.1 & 0.040 & NRM & & \\
\hline $2-3,113-116$ & 202.63 & 23.4 & 59.0 & 0.244 & 50 & 86.7 & 42.0 \\
\hline $2-4,32-35$ & 203.32 & 308.6 & 40.4 & 0.252 & 75 & 327.2 & 38.2 \\
\hline $2-5,107-109$ & 206.57 & 140.0 & -65.8 & 0.035 & NRM & & \\
\hline $3-1,23-25$ & 208.23 & 228.5 & 68.3 & 0.214 & 75 & 317.6 & 52.2 \\
\hline $3-1,136-138$ & 209.36 & 77.0 & -9.4 & 0.019 & NRM & & \\
\hline $4-1,34-37$ & 217.84 & 213.4 & 29.8 & 0.040 & NRM & & \\
\hline $4-1,139-141$ & 218.89 & 302.5 & -24.4 & 3.556 & 50 & 214.8 & -18.1 \\
\hline $4-2,133-136$ & 220.33 & 49.4 & -20.6 & 34.188 & 50 & 44.2 & -35.0 \\
\hline $4-3,55-58$ & 221.05 & 310.4 & -40.0 & 0.384 & NRM & & \\
\hline $4-4,24-26$ & 222.26 & 352.8 & -21.5 & 0.077 & & & \\
\hline $4-5,19-21$ & 224.69 & 100.3 & 48.3 & 0.038 & NRM & & \\
\hline $4-6,31-33$ & 225.31 & 308.5 & 49.8 & 0.041 & NRM & & \\
\hline $4-7,39-41$ & 226.89 & 161.5 & 30.7 & 0.064 & NRM & & \\
\hline $5-1,27-30$ & 227.27 & 114.9 & 68.4 & 0.149 & 25 & 2.5 & 40.6 \\
\hline $5-2,132-134$ & 229.82 & 32.0 & 51.3 & 0.103 & 25 & 216.0 & 70.6 \\
\hline $5-3,131-133$ & 231.31 & 315.6 & 36.0 & 0.124 & NRM & & \\
\hline $5-4,111-113$ & 232.61 & 69.4 & 52.8 & 0.131 & 25 & 74.0 & 52.0 \\
\hline $5-5,127-129$ & 234.27 & 73.5 & 54.3 & 0.063 & NRM & & \\
\hline $5-6,127-130$ & 235,77 & 156.4 & 29.2 & 0.149 & 25 & 166.7 & 57.9 \\
\hline $5-7,43-46$ & 236.43 & 66.4 & 16.7 & 0.282 & 25 & 45.6 & 45.4 \\
\hline $6-1,90-92$ & 237.40 & 7.2 & 73.6 & 0.309 & 50 & 20.9 & 40.6 \\
\hline $6-2,90-92$ & 238.90 & 296.0 & 29.3 & 0.182 & 25 & 336.6 & 25.9 \\
\hline $6-3,38-40$ & 239.88 & 28.4 & 56.5 & 0.136 & 25 & 48.7 & 49.7 \\
\hline
\end{tabular}


Table 3. (Continued).

\begin{tabular}{|c|c|c|c|c|c|c|}
\hline & & & $\begin{array}{r}\text { Before } \\
\text { magnetiz: }\end{array}$ & & & \\
\hline $\begin{array}{l}\text { Core-Sample } \\
\text { (interval in } \mathrm{cm} \text { ) }\end{array}$ & $\begin{array}{l}\text { depth } \\
\text { (m) }\end{array}$ & $\begin{array}{l}D \\
\left({ }^{\circ}\right)\end{array}$ & $\begin{array}{c}1 \\
\left({ }^{\circ}\right)\end{array}$ & $\begin{array}{l}\text { Int } \\
(\mu \mathrm{G})\end{array}$ & $\begin{array}{l}\text { field } \\
\text { (Oe) }\end{array}$ & $\begin{array}{l}\mathrm{D} \\
\left({ }^{\circ}\right)\end{array}$ \\
\hline Hole 549 (Cont.) & & & & & & \\
\hline $6-4,50-52$ & 241.50 & 192.5 & 12.9 & 0.051 & NRM & \\
\hline $6-5,21-23$ & 242.71 & 31.4 & 5.4 & 0.041 & NRM & \\
\hline $6-5,83-85$ & 243.33 & 10.6 & 19.7 & 0.151 & 25 & 356.6 \\
\hline $6-6,83-85$ & 244.83 & 347.0 & -0.7 & 0.030 & NRM & \\
\hline $7-1,13-15$ & 246.13 & 5.0 & 50.7 & 0.094 & NRM & \\
\hline $7-1,123-125$ & 247.23 & 168.3 & -37.7 & 0.132 & 25 & 225.0 \\
\hline $7-2,8-10$ & 247.58 & 343.1 & -12.7 & 0.092 & NRM & \\
\hline $7-2,100-102$ & 248.5 & 51.4 & -39.3 & 0.152 & NRM & \\
\hline $7-3,109-111$ & 250.9 & 8.6 & 40.8 & 0.975 & NRM & \\
\hline $7-5,8-10$ & 252.08 & 58.9 & -6.6 & 0.099 & NRM & \\
\hline $7-5,129-131$ & 253.29 & 143.5 & 12.1 & 0.056 & NRM & \\
\hline $8-1,127-129$ & 256.77 & 169.4 & 46.3 & 0.004 & NRM & \\
\hline $8-2,90-92$ & 257.90 & 295.4 & -43.7 & 0.442 & 25 & 229.1 \\
\hline $8-3,47-49$ & 258.97 & 341.3 & 3.6 & 0.104 & NRM & \\
\hline $8-3,99-101$ & 258.49 & 12.0 & 59.3 & 0.211 & 25 & 19.7 \\
\hline $8-4,24-26$ & 260.24 & 27.2 & -0.3 & 0.153 & 25 & 21.1 \\
\hline $8-4,138-140$ & 261.38 & 356.2 & 30.5 & 0.045 & NRM & \\
\hline $9-1,17-19$ & 265.17 & 43.8 & -41.3 & 4.146 & 150 & 52.2 \\
\hline $10-1,105-108$ & 275.55 & 305.9 & 60.0 & 10.189 & 50 & 253.1 \\
\hline $10-2,30-33$ & 276.30 & 321.2 & 76.9 & 12.296 & 50 & 49.2 \\
\hline $10-2,92-95$ & 276.92 & 133.5 & -27.5 & 10.745 & 200 & 138.0 \\
\hline $10-3,104-107$ & 278.54 & 318.2 & -7.9 & 6.021 & 50 & 313.8 \\
\hline $10-4,117-120$ & 280.17 & 6.6 & -13.6 & 3.688 & 50 & 10.5 \\
\hline $10-5,99-101$ & 281.49 & 286.9 & -37.6 & 9.769 & 50 & 278.0 \\
\hline $10-6,97-100$ & 282.97 & 70.5 & -52.2 & 11.602 & 50 & 70.8 \\
\hline $10-7,10-12$ & 283.60 & 226.8 & -11.2 & 6.873 & 50 & 224.3 \\
\hline $11-1,27-30$ & 284.27 & 78.2 & -57.7 & 7.527 & 50 & 77.4 \\
\hline $11-4,26-29$ & 288.76 & 6.1 & 14.2 & 11.530 & 50 & 7.6 \\
\hline $11-5,123-126$ & 291.23 & 11.8 & 18.8 & 3.413 & 50 & 14.3 \\
\hline $11-6,93-95$ & 292.43 & 95.3 & 19.2 & 2.249 & 50 & 102.7 \\
\hline $11-7,19-22$ & 293.19 & 85.3 & 45.8 & 18.536 & 50 & 88.4 \\
\hline $12-1,109-111$ & 294.59 & 312.2 & 25.8 & 18.428 & 50 & 310.6 \\
\hline $12-2,94-97$ & 295.94 & 211.0 & 21.0 & 5.263 & 500 & 185.7 \\
\hline $12-3,9-12$ & 296.59 & 219.1 & 36.4 & 14.783 & 100 & 217.1 \\
\hline $12-4,95-98$ & & & & 22.800 & 500 & 155.3 \\
\hline $13-1,26-29$ & & & 28.1 & 5.937 & 100 & 156.1 \\
\hline $13-2,132-135$ & 305.82 & 294.6 & 13.9 & 5.430 & 100 & 292.2 \\
\hline $13-3,27-29$ & 306.27 & 24.4 & 68.2 & 7.470 & 100 & 319.9 \\
\hline $13-4,80-83$ & 308.30 & 100.6 & 44.7 & 1.975 & 100 & 96.8 \\
\hline $13-5,44-46$ & 309.44 & 135.0 & 36.8 & 1.817 & 50 & 135.7 \\
\hline $13-6,102-105$ & 311.52 & 304.8 & 44.1 & 25.876 & 100 & 314.2 \\
\hline $13-7,12-15$ & 312.12 & 268.3 & 32.9 & 28.832 & 400 & 288.2 \\
\hline $14-1,73-76$ & 313.23 & 76.7 & 25.5 & 2.104 & 50 & 76.7 \\
\hline $14-2,128-131$ & 315.28 & 270.9 & 38.2 & 3.703 & 350 & 278.7 \\
\hline $14-3,123-125$ & 316.73 & 48.0 & 48.9 & 1.178 & 50 & 48.6 \\
\hline $14-4,16-19$ & 317.16 & 121.9 & 49.3 & 0.154 & 50 & 112.5 \\
\hline $14-4,123-125$ & 318.22 & 31.6 & 17.0 & 0.378 & 25 & 24.9 \\
\hline $14-5,75-78$ & 319.28 & 60.6 & -51.9 & 0.039 & NRM & \\
\hline $14-6,139-141$ & 321.39 & 60.2 & -44.9 & 4.695 & 50 & 62.7 \\
\hline $14-7,2-5$ & 321.52 & 251.7 & 74.0 & 7.751 & 100 & 250.8 \\
\hline $15-1,9-11$ & 322.09 & 26.6 & -7.6 & 0.558 & 50 & 27.3 \\
\hline $15-1,126-128$ & 323.26 & 299.3 & 29.8 & 0.675 & 400 & 293.4 \\
\hline $15-2,25-27$ & 323.75 & 10.5 & 18.8 & 0.674 & 100 & 4.4 \\
\hline $15-2,109-110$ & 324.58 & 229.3 & -4.5 & 2.662 & 100 & 218.6 \\
\hline $15-4,124-126$ & 327.74 & 191.2 & 4.0 & 1.267 & 100 & 206.3 \\
\hline $15-5,112-114$ & 329.12 & 275.0 & 62.0 & 0.781 & 100 & 242.0 \\
\hline $15-6,136-138$ & 330.86 & 58.6 & -26.5 & 0.447 & 100 & 119.0 \\
\hline $16-1,15-17$ & 331.65 & 7.6 & -45.5 & 0.921 & 100 & 4.0 \\
\hline $16-1,140-142$ & 332.90 & 113.0 & 33.9 & 2.241 & 300 & 130.2 \\
\hline $16-2,141-143$ & 334.41 & 157.0 & 63.8 & 1.714 & 100 & 134.9 \\
\hline $16-4,28-30$ & 336.28 & 356.1 & 73.8 & 28.795 & 500 & 253.1 \\
\hline $16-5,44-46$ & 337.94 & 165.0 & 56.7 & 8.878 & 150 & 179.1 \\
\hline $16-6,127-129$ & 340.27 & 253.5 & 46.3 & 8.474 & 150 & 228.8 \\
\hline $16-7,25-27$ & 340.75 & 182.7 & 74.5 & 8.638 & 150 & 212.7 \\
\hline $17-1,126-128$ & 342.26 & 284.3 & 11.2 & 1.592 & 300 & 288.3 \\
\hline $17-2,130-132$ & 343.80 & 193.4 & -11.6 & 1.234 & 100 & 179.5 \\
\hline $17-3,118-121$ & 345.18 & 113.1 & -52.9 & 1.584 & 100 & 34.2 \\
\hline $17-4,101-103$ & 346.51 & 307.0 & -14.0 & 3.610 & 100 & 312.2 \\
\hline $17-5,113-115$ & 348.13 & 145.0 & -10.3 & 1.475 & 100 & 154.6 \\
\hline $17-6,136-138$ & 349.86 & 144.7 & -11.2 & 14.094 & 100 & 143.9 \\
\hline $18-1,103-105$ & 351.53 & 149.2 & 14.6 & 3.675 & 200 & 145.0 \\
\hline $18-2,21-23$ & 352.21 & 20.2 & 16.3 & 1.088 & 150 & 19.1 \\
\hline $18-2,101-103$ & 353.01 & 208.3 & 23.2 & 10.492 & 200 & 210.5 \\
\hline $18-3,31-33$ & 353.81 & 30.0 & 26.1 & 7.847 & 200 & 39.7 \\
\hline $18-3,91-93$ & 354.41 & 44.2 & 9.9 & 2.11 & 200 & 49.1 \\
\hline $19-1,46-48$ & 360.48 & 223.1 & -20.3 & 0.581 & 50 & 217.7 \\
\hline $19-2,55-58$ & 362.05 & 247.2 & -59.0 & 1.718 & 50 & 257.0 \\
\hline $19-3,116-118$ & 364.16 & 6.6 & -6.8 & 0.610 & 50 & 352.9 \\
\hline $19-4,12-14$ & 364.62 & 244.0 & 22.8 & 0.210 & 50 & 231.4 \\
\hline $19-4,115-117$ & 365.65 & 205.3 & -61.0 & 0.109 & NRM & \\
\hline $19-5,26-28$ & 366.26 & 22.4 & -25.4 & 0.088 & NRM & \\
\hline $20-1,10-12$ & 369.60 & 27.6 & -12.2 & 2.262 & 50 & 31.0 \\
\hline $20-2,43-45$ & 371.43 & 117.0 & 45.9 & 0.273 & 50 & 84.0 \\
\hline $20-3,38-40$ & 372.88 & 106.6 & 36.5 & 0.112 & 25 & 83.9 \\
\hline $20-3,142-144$ & 373.92 & 349.2 & -10.1 & 0.160 & 50 & 355.5 \\
\hline $20-4,31-33$ & 374.31 & 88.8 & 27.0 & 0.454 & 25 & 50.2 \\
\hline $20-5,9-11$ & 375.69 & 213.6 & 42.1 & 0.448 & 25 & 229.3 \\
\hline $20-6,15-17$ & 777.15 & 220.0 & 65.6 & 0.092 & NRM & \\
\hline $21-1,114-116$ & 380.14 & 37.2 & 63.7 & 1.766 & 50 & 282.9 \\
\hline
\end{tabular}


Table 3. (Continued).

\begin{tabular}{|c|c|c|c|c|c|c|}
\hline & Sub-bottom & & $\begin{array}{r}\text { Before } \\
\text { nagnetiz }\end{array}$ & & & \\
\hline $\begin{array}{c}\text { Core-Sample } \\
\text { (interval in cm) }\end{array}$ & $\begin{array}{l}\text { depth } \\
\text { (m) }\end{array}$ & $\begin{array}{l}\mathrm{D} \\
\left({ }^{\circ}\right)\end{array}$ & $\begin{array}{l}1 \\
\left({ }^{\circ}\right)\end{array}$ & $\begin{array}{c}\text { Int } \\
(\mu \mathrm{G})\end{array}$ & $\begin{array}{l}\text { field } \\
\text { (Oe) }\end{array}$ & $\begin{array}{c}\text { D } \\
\left({ }^{\circ}\right)\end{array}$ \\
\hline Hole 549 (Cont.) & & & & & & \\
\hline $21-2,24-26$ & 380.74 & 48.3 & -16.0 & 0.215 & 25 & 207.5 \\
\hline $21-2,83-85$ & 381.33 & 211.4 & -6.5 & 0.992 & so & 203.0 \\
\hline $21-3,118-120$ & 393.18 & 348.8 & 42.7 & 6.565 & 50 & 330.8 \\
\hline $22-1,52-54$ & 389.02 & 309.6 & 64.4 & 5.995 & 150 & 212.5 \\
\hline $22-1,104-106$ & 389.54 & 229.5 & -16.5 & 6.212 & 200 & 204.8 \\
\hline $22-2,32-34$ & 390.32 & 212.8 & 62.7 & 13.192 & 300 & 219.5 \\
\hline $22-3,31-33$ & 391.81 & 343.4 & 53.3 & 3.561 & 200 & 343.5 \\
\hline $22-3,131-133$ & 392.81 & 157.2 & 73.3 & 3.137 & 200 & 35.5 \\
\hline $22-4,46-48$ & 393.46 & 147.5 & 57.2 & 4.160 & 150 & 153.6 \\
\hline $22-5,24-26$ & 394.74 & 149.9 & 66.0 & 3.900 & 150 & 15.3 \\
\hline $22-5,55-57$ & 395.05 & 207.2 & 76.8 & 4.978 & 200 & 194.4 \\
\hline $23-1,23-25$ & 398.23 & 18.3 & 58.9 & 14.204 & 150 & 57.7 \\
\hline $23-2,140-143$ & 400.90 & 24.3 & 56.2 & 11.201 & 150 & 65.0 \\
\hline $23-3,12-15$ & 401.12 & 236.9 & 60.1 & 17.074 & 150 & 266.8 \\
\hline $23-3,145-147$ & 402.45 & 356.7 & 40.3 & 5.741 & 100 & 2.9 \\
\hline $23-4,7-10$ & 402.57 & 20.5 & 49.8 & 18.407 & 100 & 54.5 \\
\hline $23-4,115-117$ & 403.65 & 293.0 & 41.7 & 5.282 & 150 & 305.0 \\
\hline $23-5,3-6$ & 404.13 & 204.8 & 32.1 & 13.563 & 200 & 213.8 \\
\hline $25-5,135-138$ & 405.35 & 25.8 & 77.1 & 6.713 & 200 & 285.8 \\
\hline $24-1,9-12$ & 407.59 & 337.7 & -27.7 & 2.538 & 100 & 320.3 \\
\hline $24-1,144-147$ & 408.94 & 339.6 & 50.3 & 5.047 & 100 & 348.1 \\
\hline $24-2,5-8$ & 409.05 & 224.5 & 46.9 & 5.383 & 100 & 220.0 \\
\hline $24-3,7-10$ & 410.57 & 294.8 & 57.2 & 3.790 & 100 & 288.6 \\
\hline $25-1,55-58$ & 417.55 & 121.3 & 62.2 & 15.832 & 100 & 127.4 \\
\hline $25-2,5-8$ & 418.55 & 308.5 & 57.2 & 28.718 & 100 & 284.7 \\
\hline $26-1,21-24$ & 426.71 & 265.0 & 42.6 & 0.485 & 100 & 274.5 \\
\hline $27-1,7-10$ & 436.07 & 353.2 & -0.2 & 1.395 & 100 & 305.0 \\
\hline $28-1,36-39$ & 445.86 & 302.9 & 64.1 & 6.923 & 100 & 295.2 \\
\hline $28-1,101-103$ & 448.01 & 336.0 & 54.5 & 97.37 & 100 & 328.4 \\
\hline $28-3,35-38$ & 448.85 & 171.7 & 60.3 & 62.027 & 100 & 177.3 \\
\hline $29-1,5-8$ & 455.05 & 173.7 & 58.1 & 54.863 & 100 & 175.6 \\
\hline $35-1,75-77$ & $\$ 12.75$ & 129.9 & 45.2 & 0.354 & NRM & \\
\hline $36-1,5-7$ & 521.55 & 316.1 & 50.9 & 0.148 & NRM & \\
\hline $37-1,41-43$ & 522.91 & 124.6 & 23.3 & 0.972 & 100 & 120.0 \\
\hline $37-1,106-108$ & 523.56 & 224.3 & 49.5 & 0.503 & 100 & 218.1 \\
\hline $37-2,4-7$ & 524.04 & 63.3 & -38.7 & 0.451 & 50 & 70.8 \\
\hline $38-1,35-38$ & 531.55 & 181.7 & -63.6 & 0.154 & 100 & 302.0 \\
\hline $39-1,24-27$ & 540.74 & 277.3 & 36.5 & 0.020 & NRM & \\
\hline $40-1,22-25$ & 550.22 & 89.3 & 53.0 & 0.092 & NRM & \\
\hline $42-1,52-55$ & 569.52 & 271.5 & 61.4 & 0.125 & NRM & \\
\hline $42-2,12-15$ & 570.62 & 139.6 & 4.9 & 0.218 & 50 & 116.4 \\
\hline $42-3,13-16$ & 571.13 & 22.1 & 17.9 & 0.327 & 50 & 358.8 \\
\hline $43-1,92-94$ & 579.42 & 328.9 & 60.7 & 0.824 & so & 343.2 \\
\hline $43-2,29-31$ & 580.29 & 22.7 & 59.5 & 0.640 & so & 1.0 \\
\hline $43-3,45-47$ & 581.95 & 102.8 & 51.4 & 1.110 & 50 & 105.8 \\
\hline $43-4,68-70$ & 583.63 & 148.7 & 50.7 & 1.288 & 50 & 151.0 \\
\hline $44-1,32-34$ & 588.32 & 183.0 & 59.9 & 0.234 & 50 & 182.9 \\
\hline $44-2,53-55$ & 590.03 & 222.4 & 59.2 & 0.813 & 50 & 203.5 \\
\hline $44-3,4-6$ & 591.04 & 317.0 & 51.4 & 0.550 & 50 & 306.1 \\
\hline $44-4,121-123$ & 593.71 & 230.7 & 62.1 & 1.280 & so & 231.9 \\
\hline $45-1,67-69$ & 598.17 & 254.9 & 52.3 & 1.564 & 50 & 257,1 \\
\hline $45-2,7-9$ & 599.07 & 82.7 & 52.0 & 1.103 & 50 & 82.8 \\
\hline $45-3,15-17$ & 600.65 & 235.7 & 50.0 & 1.663 & 50 & 234.6 \\
\hline $45-4,40-42$ & 602.40 & 262.8 & 51.4 & 1.743 & so & 253.1 \\
\hline $45-5,15-17$ & 603.65 & 182.7 & 55.5 & 1.649 & 50 & 186.8 \\
\hline $46-1,59-61$ & 607.59 & 97.1 & 50.7 & 4.272 & 50 & 103.3 \\
\hline $46-2,62-64$ & 609.12 & 105.4 & 49.9 & 2.237 & 50 & 107.7 \\
\hline $46-3,51-53$ & 610.51 & 70.8 & 58.7 & 2.147 & 50 & 71.5 \\
\hline $46-4,66-68$ & 612.16 & 74.2 & 58.2 & 2.042 & 50 & 71.6 \\
\hline $47-1,110-112$ & 617.60 & 83.3 & 67.9 & 5.725 & 50 & 85.2 \\
\hline $47-2,20-22$ & 618.20 & 4.5 & 49.8 & 1.401 & 50 & 350.4 \\
\hline $47-3,53-55$ & 620.03 & 206.4 & 54.0 & 1.721 & 50 & 225.0 \\
\hline $47-4,10-12$ & 621.10 & 63.7 & 65.1 & 0.943 & 50 & 67.5 \\
\hline $47-4,97-99$ & 621.97 & 203.2 & 65.1 & 1.453 & 200 & 205.1 \\
\hline $47-5,16-18$ & 622.66 & 156.3 & 61.3 & 2.535 & 200 & 158.3 \\
\hline $48-1,15-17$ & 626.15 & 161.9 & -61.3 & 1.826 & 50 & 226.2 \\
\hline $52-1,27-29$ & 664.27 & 325.6 & -1.6 & 0.760 & 75 & 290.2 \\
\hline $54-1,12-14$ & 683.12 & 2.2 & 63.2 & 4.419 & 75 & 358.5 \\
\hline $54-2,80-82$ & 685.30 & 93.3 & 64.0 & 2.821 & 75 & 95.3 \\
\hline $54-3,109-111$ & 687.09 & 66.8 & 57.6 & 1.119 & 50 & 52.4 \\
\hline $54-4,32-34$ & 687.82 & 274.2 & 72.0 & 1.353 & 50 & 263.2 \\
\hline $55-1,29-31$ & 692.29 & 3.8 & 55.6 & 0.674 & 50 & 359.9 \\
\hline $55-2,16-18$ & 693.66 & 103.3 & 56.3 & 1.263 & 50 & 111.0 \\
\hline $55-3,135-137$ & 696.35 & 337.2 & 55.9 & 18.036 & 50 & 332.1 \\
\hline $55-4,67-69$ & 697.17 & 29.9 & 51.1 & 10.214 & 50 & 44.3 \\
\hline $55-5,75-77$ & 698.75 & 295.6 & 64.3 & 1.586 & 100 & 295.0 \\
\hline $56-1,139-141$ & 702.39 & 177.8 & 77.6 & 2.161 & 100 & 183.3 \\
\hline $56-2,49-51$ & 702.99 & 245.5 & 52.9 & 2.816 & 100 & 245.0 \\
\hline $56-3,91-93$ & 704.91 & 8.7 & 53.0 & 20.059 & 100 & 7.9 \\
\hline $56-4,35-37$ & 705.85 & 308.4 & 70.6 & 1.804 & 100 & 307.1 \\
\hline $56-5,30-32$ & 707.30 & 348.0 & 54.2 & 16.428 & 100 & 345.3 \\
\hline $57-1,22-24$ & 710.22 & 195.7 & 71.3 & 22.861 & 100 & 167.8 \\
\hline $57-2,22-25$ & 711.75 & 299.1 & 51.2 & 16.844 & 100 & 290.5 \\
\hline $57-3,24-26$ & 713.24 & 214.7 & 59.1 & 40.611 & 100 & 205.4 \\
\hline $57-4,52-54$ & 716.02 & 92.9 & 52.2 & 19.362 & 100 & 94.3 \\
\hline $57-5,8-10$ & 717.08 & 149.9 & 60.5 & 8.972 & 100 & 155.2 \\
\hline $58-1,107-109$ & 720.07 & 121.6 & 63.6 & 38.502 & 100 & 133.6 \\
\hline $58-2,18-20$ & 620.68 & 245.2 & 55.7 & 69.032 & 100 & 240.2 \\
\hline $58-3,45-47$ & 722.45 & 23.0 & 53.0 & 35.461 & 100 & 26.6 \\
\hline
\end{tabular}


Table 3. (Continued).

\begin{tabular}{|c|c|c|c|c|c|c|c|c|c|}
\hline \multirow[b]{2}{*}{$\begin{array}{l}\text { Core-Sample } \\
\text { (interval in } \mathrm{cm} \text { ) }\end{array}$} & \multirow{2}{*}{$\begin{array}{l}\text { Sub-bottom } \\
\text { depth } \\
\text { (m) }\end{array}$} & \multicolumn{3}{|c|}{$\begin{array}{c}\text { Before } \\
\text { demagnetization }\end{array}$} & \multirow{2}{*}{$\begin{array}{l}\text { Demagnetization } \\
\text { field } \\
(\mathrm{Oe}) \\
\end{array}$} & \multicolumn{4}{|c|}{$\begin{array}{c}\text { After } \\
\text { demagnetization }\end{array}$} \\
\hline & & $\begin{array}{l}D \\
\left({ }^{\circ}\right)\end{array}$ & $\begin{array}{l}1 \\
\left({ }^{\circ}\right)\end{array}$ & $\begin{array}{c}\text { Int } \\
(\mu \mathrm{G})\end{array}$ & & $\begin{array}{l}\mathrm{D} \\
\left({ }^{\circ}\right)\end{array}$ & $\begin{array}{c}\mathrm{I} \\
\left(^{\circ}\right)\end{array}$ & $\begin{array}{c}\text { Int } \\
(\mu \mathrm{G})\end{array}$ & Polarity \\
\hline \multicolumn{10}{|l|}{ Hole 549 (Cont.) } \\
\hline $58-4,22-24$ & 723.72 & 280.3 & 57.6 & 80.158 & 100 & 277.8 & 53.3 & 54.604 & N \\
\hline $58-5,19-22$ & 725.19 & 254.3 & 60.1 & 10.078 & 100 & 251.3 & 56.4 & 4.324 & $\mathrm{~N}$ \\
\hline $58-6,19-21$ & 725.69 & 103.4 & 47.9 & 113.185 & 100 & 105.6 & 45.8 & 76.069 & N \\
\hline $58-7,15-17$ & 728. & 275.3 & 44.6 & 87,493 & 100 & 268.6 & 43.8 & 58.705 & $\mathrm{~N}$ \\
\hline $59-1,16-18$ & 728.16 & 328.8 & 56.1 & 62.597 & 100 & 323.6 & 52.5 & 30.187 & $\mathrm{~N}$ \\
\hline $59-2,16-17$ & 729.66 & 13.5 & 71.3 & 3.235 & 100 & 13.3 & 53.4 & 0.545 & $\mathrm{~N}$ \\
\hline $59-3,11-13$ & 731.11 & 141.0 & 59.5 & 76.498 & 100 & 150.1 & 63.2 & 19.118 & $\mathrm{~N}$ \\
\hline $60-1,109-111$ & 738.09 & 150.6 & 56.6 & 2.018 & 100 & 152.7 & 51.7 & 1.061 & \\
\hline $60-2,98-100$ & 739.98 & 39.5 & 60.6 & 1.310 & 100 & 83.5 & 63.1 & 0.631 & $\mathrm{~N}$ \\
\hline $60-3,33-35$ & 740.33 & 115.3 & 64.8 & 1.999 & 100 & 95.9 & 60.8 & 1.882 & N \\
\hline $60-4,36-38$ & 741.86 & 244.9 & 59.5 & 4.092 & 100 & 259.0 & 72.6 & 1.632 & $\mathrm{~N}$ \\
\hline $60-5,31-33$ & 743.31 & 21.0 & 53.8 & 27.247 & 100 & 23.0 & $\$ 1.7$ & 14.311 & $\mathrm{~N}$ \\
\hline $60-6,37-39$ & 745.87 & 288.6 & 51.6 & 13.822 & 100 & 289.1 & 51.7 & 5.552 & $\mathrm{~N}$ \\
\hline $61-1,25-27$ & 746.25 & 96.9 & 66.3 & 0.230 & 100 & 17.9 & 70.1 & 0.327 & $\mathrm{~N}$ \\
\hline $61-2,49-51$ & 747.99 & 351.1 & 55.1 & 1.780 & 100 & 340.9 & 53.7 & 1.207 & $\mathrm{~N}$ \\
\hline $61-3,16-18$ & 749.16 & 342.6 & 53.6 & 0.603 & 100 & 312.6 & 48.4 & 1.115 & N \\
\hline $73-1,95-97$ & 807.45 & 94.2 & 48.9 & 0.347 & 50 & 81.9 & 53.9 & 0.775 & N \\
\hline $73-2,87-89$ & 808.87 & 236.1 & 47.0 & 1.934 & 50 & 226.9 & 60.0 & 1.659 & $\mathrm{~N}$ \\
\hline $74-1,14-17$ & 811.14 & 264.2 & 61.3 & 1.535 & 50 & 246.6 & 56.9 & 0.989 & $\mathrm{~N}$ \\
\hline $74-2,43-45$ & 812.93 & 232.9 & 55.5 & 1.242 & 100 & 234.4 & 53.5 & 1.584 & $\mathrm{~N}$ \\
\hline $74-3,121-124$ & 815.21 & 233.4 & 58.6 & 0.768 & 100 & 211.7 & 58.4 & 0.512 & $\mathrm{~N}$ \\
\hline $75-1,128-131$ & 817.25 & 156.4 & 61.9 & 1.414 & 100 & 167.0 & 58.8 & 1.225 & $\mathrm{~N}$ \\
\hline $75-2,124-126$ & 818.74 & 313.8 & 68.2 & 0.633 & 100 & 200.4 & 76.9 & 0.336 & $\mathrm{~N}$ \\
\hline $75-3,7-10$ & 819.07 & 67.0 & 75.3 & 0.337 & 100 & 64.0 & 32.2 & 0.113 & $\mathrm{~N}$ \\
\hline $76-1,102-104$ & 826.02 & 19.6 & 61.0 & 5.593 & 100 & 15.7 & 62.0 & 2.792 & $\mathrm{~N}$ \\
\hline $76-2,134-136$ & 827.84 & 306.5 & 72.5 & 4.449 & 100 & 308.9 & 66.4 & 2.180 & $\mathrm{~N}$ \\
\hline $76-3,7-9$ & 828.07 & 291.4 & 77.4 & 2.572 & 100 & 294.5 & 60.9 & 2.265 & $\mathrm{~N}$ \\
\hline $78-1,86-88$ & 843.86 & 344.7 & 59.7 & 12.276 & 100 & 347.4 & 55.5 & 7.478 & $\mathrm{~N}$ \\
\hline $79-1,61-63$ & 852.61 & 146.6 & 62 & 2.776 & 100 & 151.8 & 58.2 & 2.610 & $\mathrm{~N}$ \\
\hline $80-1,64-$ & 861.64 & 174.2 & 5. & 0.951 & 50 & 162.9 & 59.8 & 1.359 & N \\
\hline $80-2,79-81$ & 863.29 & 100.6 & 22.1 & 0.808 & 50 & 104.1 & 20.6 & 0.763 & N \\
\hline $81-1,135-137$ & 871.35 & 147.2 & 65 & 2.090 & 50 & 152,0 & 54.8 & 1.385 & $\mathrm{~N}$ \\
\hline $81-2,44$ & 871.94 & 271.8 & si & 0.548 & 50 & 262.9 & 54.1 & 0.535 & $\mathrm{~N}$ \\
\hline $82-1,33-35$ & 874.83 & 323.4 & 42.4 & 0.293 & 50 & 11.0 & 68.2 & 0.323 & $\mathrm{~N}$ \\
\hline $82-2,51-54$ & 875.01 & 279.1 & -15.3 & & 150 & 261.4 & -1.5 & 0.335 & $\mathrm{R}$ ? \\
\hline $83-1,13-15$ & 879.13 & 216.2 & 66.6 & 0.125 & 100 & 230.8 & 51.8 & 0.207 & $\mathrm{~N}$ \\
\hline $83-2,69-71$ & 881.19 & 248.3 & 84.3 & 1.327 & so & 229.1 & 79.4 & 1.237 & $\mathrm{~N}$ \\
\hline $83-3,9-11$ & 882.09 & 290.3 & -59.6 & 0.496 & 75 & 273.6 & -65.0 & 0.083 & $\mathbf{R}$ \\
\hline $84-1,56-59$ & 884.56 & 149.2 & 72.4 & 0.863 & 150 & 151.0 & 62.2 & 0.772 & $\mathrm{~N}$ \\
\hline $84-2,82-84$ & 886.32 & 71.3 & 28.1 & 1.584 & 300 & 66.7 & 34.0 & 1.339 & $\mathrm{~N}$ \\
\hline $85-1,19-21$ & 888.19 & 219.9 & 56.4 & 3.415 & 100 & 221.2 & 54.5 & 2.032 & $N$ \\
\hline $85-2,49-51$ & 889.97 & 118.7 & 54.3 & 1.197 & 100 & 136.0 & 55.6 & 0.673 & $\mathrm{~N}$ \\
\hline $85-3,9-11$ & 891.09 & 231.2 & 58.6 & 2.858 & 100 & 240.8 & 50.9 & 1.897 & $\mathrm{~N}$ \\
\hline $86-1,16-18$ & 897,16 & 209.3 & 67.4 & 0.619 & 100 & 166.5 & 47.5 & 0.354 & $\mathrm{~N}$ \\
\hline $86-2,27-29$ & 898.77 & 211.0 & 76.2 & 0.259 & 25 & 140.5 & 76.8 & 0.205 & $\mathrm{~N}$ \\
\hline $86-3,25-27$ & 900.25 & 178.2 & 47.0 & 0.095 & 25 & 228.1 & 60.6 & 0.175 & $\mathrm{~N}$ \\
\hline $87-2,37-39$ & 907.37 & 224.9 & 53.5 & 4.133 & 50 & 234.1 & 58.1 & 4.229 & N \\
\hline $87-3,9-11$ & 909.9 & 11.8 & & 1.272 & so & 9.2 & 55.2 & 1.222 & $\mathrm{~N}$ \\
\hline $88-1,22-25$ & 910.72 & 179.9 & 52.3 & 2.017 & 50 & 177.7 & 53.9 & 1.551 & $\mathrm{~N}$ \\
\hline $88-2,99-102$ & 912.99 & 245.3 & 60.2 & 0.082 & NRM & & & & N \\
\hline $88-3,37-40$ & 913. & 252.1 & 69 & 0.1 & 100 & 186.2 & 63.4 & 0.277 & N \\
\hline $89-1,88$ & 920. & 138.2 & & & 100 & 137.6 & 51.9 & 0.663 & $N$ \\
\hline $89-2,12$ & 922. & & 56 & & 50 & 353.0 & 51.4 & 0.691 & $\mathrm{~N}$ \\
\hline 9 & & & -2 & & 75 & & 33.3 & 0.395 & $\mathrm{~N}$ ? \\
\hline & & & 44 & & so & 340.6 & 61.2 & 0.942 & N \\
\hline 9 & & 357 & & & 50 & 2.6 & 63.4 & 0.359 & $\mathrm{~N}$ \\
\hline & & & & & NRM & & & & $N$ \\
\hline & & & & & 50 & 355.8 & 69.6 & 0.35 & $N$ \\
\hline $92-1,50-$ & & & 43.9 & & NRM & & & & $N$ \\
\hline $93-1,46-48$ & & 305.8 & 23.6 & 0.280 & 50 & 314.5 & 47.3 & 0.338 & N \\
\hline $94-1,20-22$ & 964.70 & 80.3 & 12.4 & 0.176 & 50 & 108.6 & 68.4 & 0.676 & $\mathrm{~N}$ \\
\hline
\end{tabular}

Note: Symbols and abbreviations as in Table 1.

The paleomagnetic data show no evidence of hiatuses in the Upper Cretaceous to Paleocene section of Hole $550 \mathrm{~B}$, but biostratigraphic studies suggest that the lower part of Anomaly 33 and the upper part of the long Cretaceous normal polarity interval may be absent. Three short reversals have been detected in Cores 23 to 25 that may represent the mixed polarity interval of late Albian age identified by van Hinte (1976) and recognized at DSDP Site 263 off Western Australia (Green and Brecher, 1974 and Jarrard, 1974) and Sites 400 and 402 in the Bay of Biscay (Hailwood, 1979). The identification of these reversals in the sediments directly overlying basement is of particular significance in view of the age of the oldest sediments at this site. The paleomagnetic evidence therefore suggests a late Albian age for these sediments. This is in broad agreement with the biostratigraphic studies.

\section{Site $\mathbf{5 5 1}$}

Site 551 was washed to $100 \mathrm{~m}$ and then rotary drilled. The sediments recovered were a condensed sequence of early Eocene calcareous muds resting uncomfortably on Late Cretaceous nannofossil chalks. The results from this hole are listed in Table 5 . Figure 18 shows the downhole variation in inclination and the polarity reversal sequence deduced for the hole. Three normal polarity intervals occur in the Cretaceous part of the sequence. The upper two, above the Cenomanian/Campanian unconformity, probably represent Anomalies 32 and 33, the lowest one Anomaly 34 . 

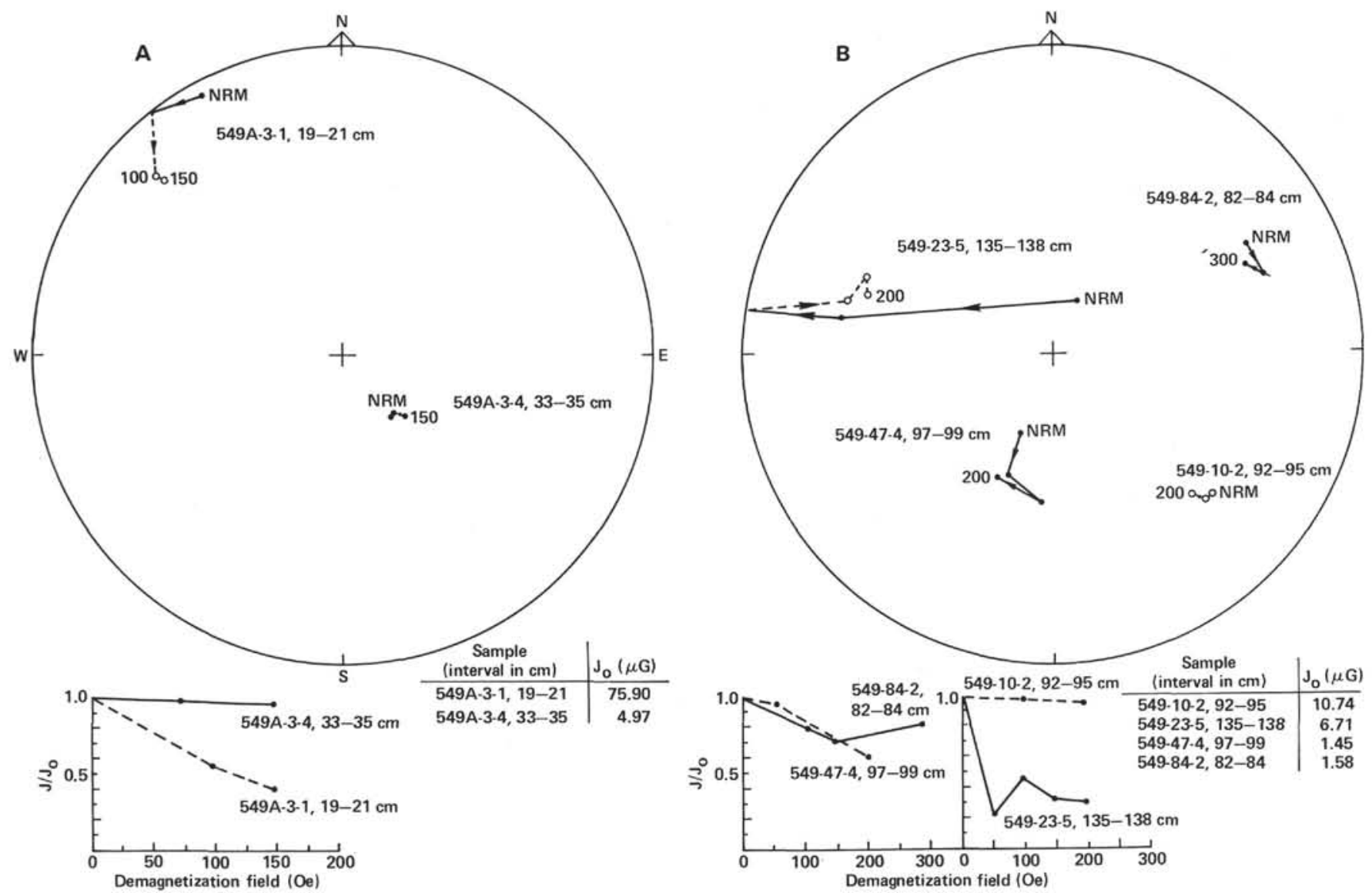

Figure 9. Response of typical samples from Site 549 to af demagnetization. A. Samples from Hole 549A. B. Samples from Hole 549. Symbols as in Fig. 2.

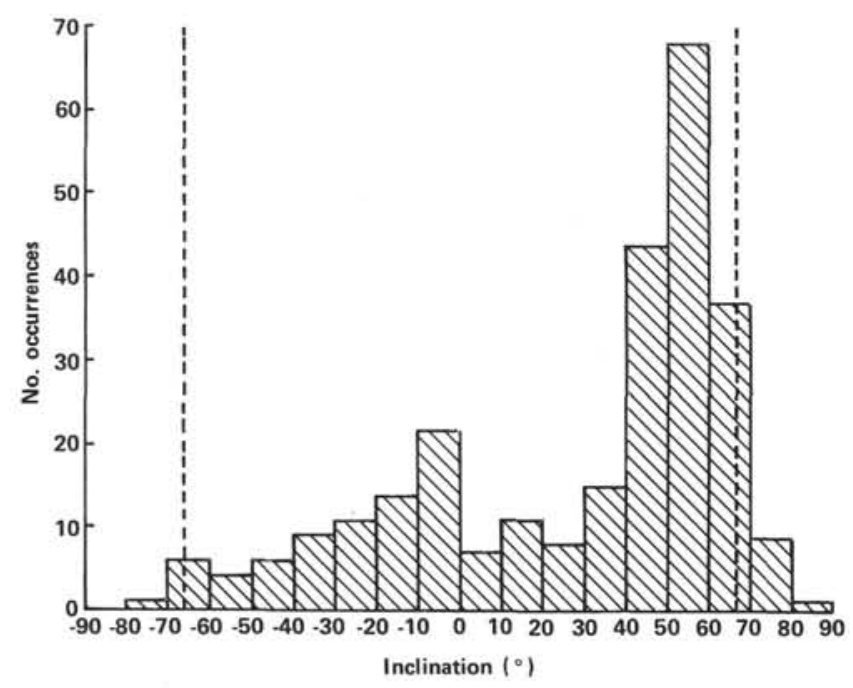

Figure 10. Histogram of magnetic inclination values for Hole 549. Dashed lines represent present-day theoretical geocentric dipole values.

\section{CONCLUSIONS}

Magnetostratigraphic studies for the sites drilled across the Goban Spur have enabled polarity reversal sequences to be established. The correlation of reversal sequenc- es with standard Cenozoic and Cretaceous polarity time scales permits the refinement and enhancement of the biostratigraphic dating of the sediments.

In this study Site 549 provided the section most suitable for paleomagnetic studies, because an almost complete sequence of upper Paleocene to upper Oligocene pelagic sediments containing rich faunal assemblages was recovered. Unfortunately, the upper Eocene and Oligocene sediments were too weakly magnetized for an unequivocal magnetostratigraphy to be determined, but good results were obtained from the middle Eocene and upper Paleocene section. Figure 19 shows a summary of the paleomagnetic and biostratigraphic data for Sites 548,549 , and 550 , together with a magnetostratigraphic correlation. Although the section at Site 549 appeared to be complete from biostratigraphic evidence, studies of the ash layers (Knox, this volume) indicate a hiatus at the NP9/NP10 boundary. The normal polarity interval at the NP9/NP8 boundary at Site 549 is rather low to be Anomaly 25, which may be absent as a result of the hiatus. If it is absent, the correlation shown in Figure 19 between the normal polarity intervals assigned to Anomaly 25 at Sites 549 and 550 would be incorrect.

Figure 19 also illustrates generally better agreement between the magnetostratigraphy and the nannoplankton zones than the foraminifer zones. For example, the 


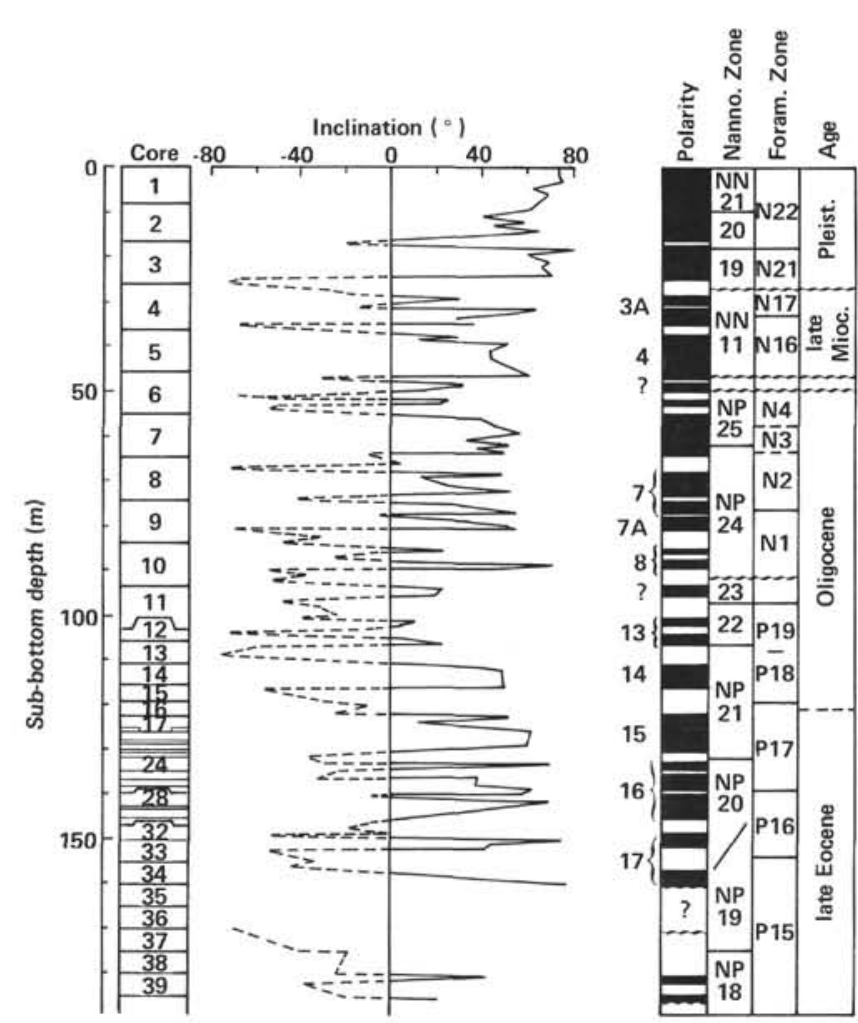

Figure 11. Downhole variation of magnetic inclination and inferred polarity reversal sequence for Hole 549A.

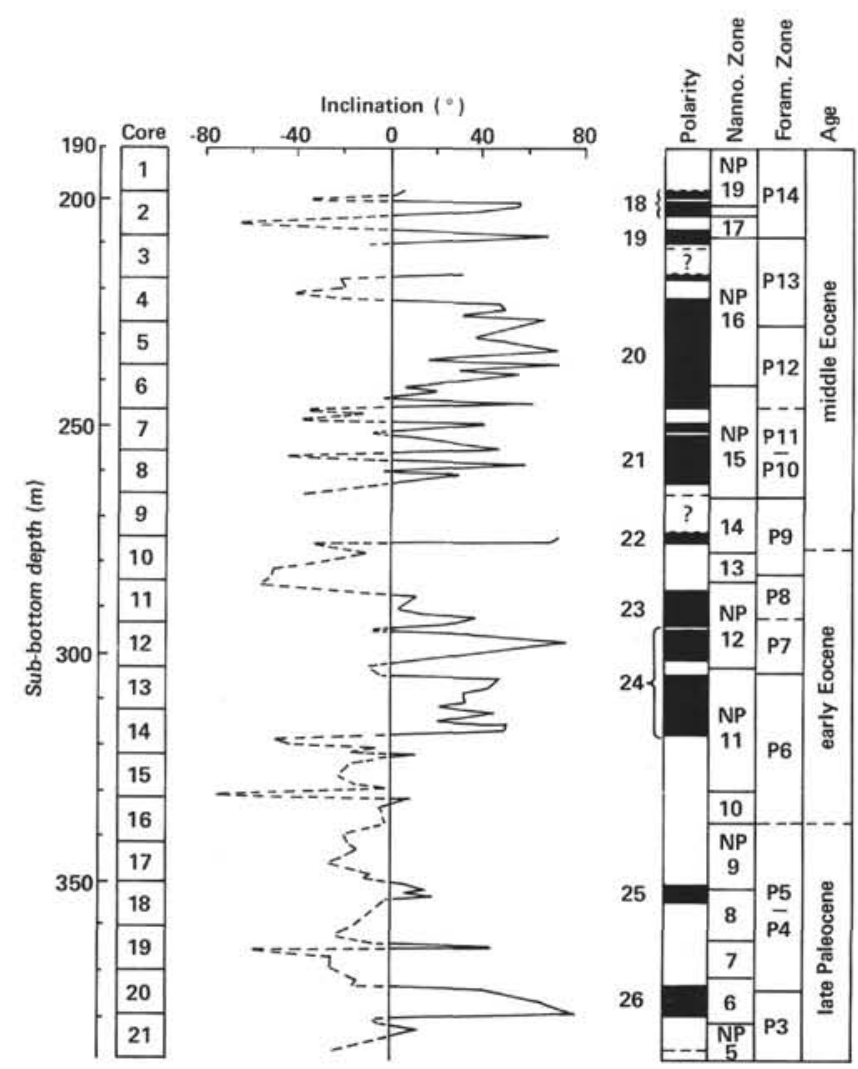

Figure 12. Variation of magnetic inclination with depth and inferred polarity reversal sequence for the late Paleocene to middle Eocene sediments from Hole 549.

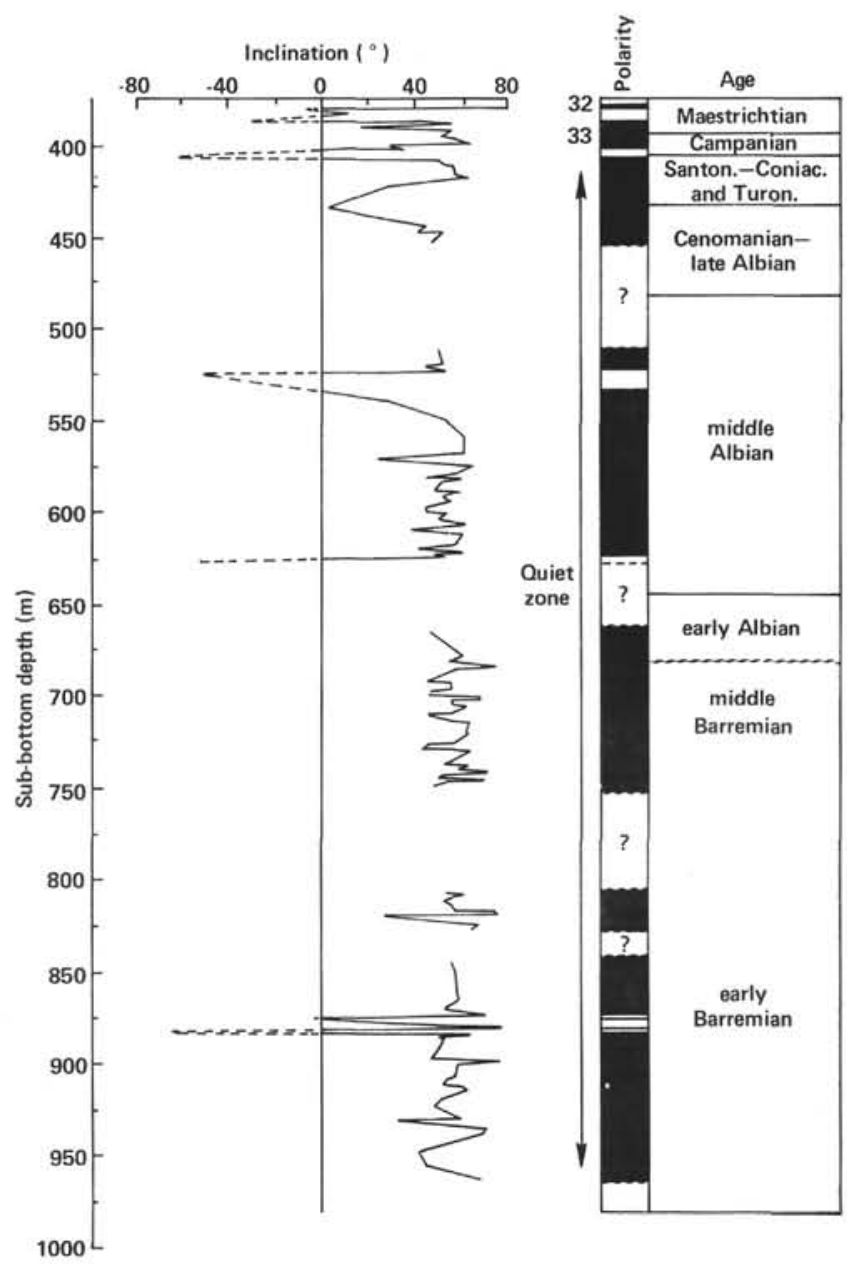

Figure 13. Downhole variation of magnetic inclination and inferred polarity reversal sequence for the Cretaceous sediments from Hole 549.

normal polarity interval assigned to Anomaly 23 lies within NP12 at both Sites 549 and 550, but it lies within P8/P7 at Site 549 and P9/P8 at Site 550.

An important result of the paleomagnetic studies is that changes in the values of NRM intensity and magnetic susceptibility correlated with changes in these values at other sites. At Sites 548 and 549 a significant downhole increase in the values of NRM intensity and magnetic susceptibility was noted in nannoplankton Zone NP14, which agrees with the results from Holes $400 \mathrm{~A}$ and 401 of Leg 48. This increase is of particular interest in view of the results of the magnetic fabric studies on these sediments (Hailwood and Folami, this volume), which suggest that a change in sediment transport processes occurred at this time.

The paleomagnetic studies are also of value in assigning absolute ages to the sediments. The assignment of absolute ages is dealt with in Snyder et al. (this volume).

\section{ACKNOWLEDGMENTS}

I would like to thank all the people who have assisted me during the course of this research, particularly Ernie Hailwood for his helpful advice and encouragement. I am grateful to the shipboard scientific and technical party, especially to Carla Müller and Scott Snyder for access to their paleontological results. 


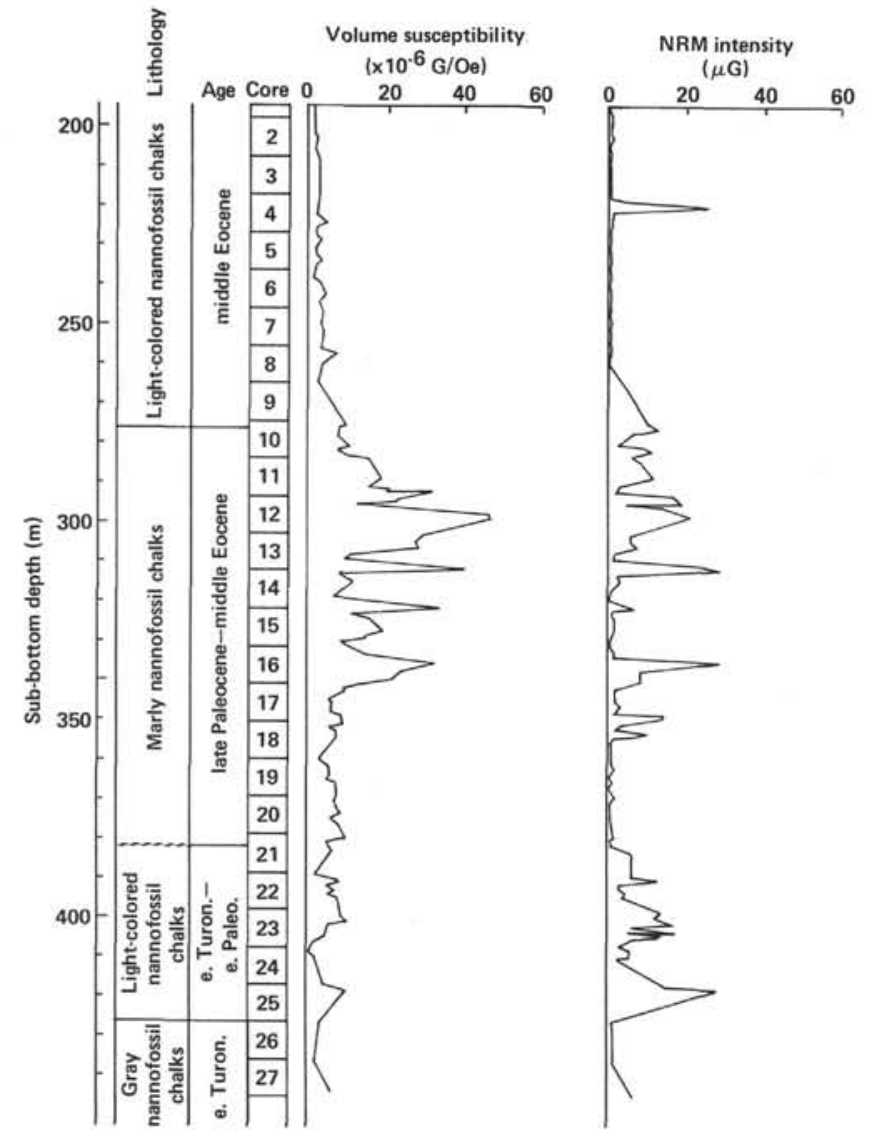

Figure 14. Downhole variation of magnetic susceptibility and NRM intensity, Hole 549.
I would like to thank Joan Wedge and Jean Watson for typing this manuscript. Financial assistance from the Natural Environment Research Council is gratefully acknowledged.

\section{REFERENCES}

Green, K. A., and Brecher, A., 1974. Preliminary paleomagnetic results for sediments from Site 263, Leg 27. In Veevers, J. J., Heirtzler, J. R., et al., Init. Repts. DSDP, 27: Washington (U.S. Govt. Printing Office), 405-413.

Hailwood, E. A., 1979. Paleomagnetism of late Mesozoic to Holocene sediments from the Bay of Biscay and Rockall Plateau, drilled on IPOD Leg 48. In Montadert, L., Roberts, D. G., et al., Init. Repts. DSDP, 48: Washington (U.S. Govt. Printing Office), 305-339.

Hailwood, E. A., Bock, W., Costa, L., Dupeuble, P. A., Müller, C., and Schnitker, D., 1979. Chronology and biostratigraphy of Northeast Atlantic sediments, DSDP Leg 48. In Montadert, L., Roberts, D. G., et al., Init. Repts. DSDP, 48: Washington (U.S. Govt. Printing Office), 1119-1141.

Jarrard, R. D., 1974. Paleomagnetism of some Leg 27 sediment cores. In Veevers, J. J., Heirtzler, J. R., et al., Init. Repts. DSDP, 27: Washington (U.S. Govt. Printing Office), 415-423.

LaBrecque, J. L., Kent, D. V., and Cande, S. C., 1977. Revised magnetic polarity time scale for the Late Cretaceous and Cenozoic time. Geology, 5:330-335.

Lowrie, W., and Alvarez, W., 1981. One hundred million years of geomagnetic polarity history. Geology, 9:392-397.

Molyneux, L., 1971. A compiete results magnetometer for measuring the remanent magnetization of rocks. Geophys. J. R. Astron. Soc., 24:429-433.

Ness, G., Levi, S., and Couch, R., 1980. Marine magnetic anomaly time scales for the Cenozoic and Late Cretaceous. A précis, critique and synthesis. Rev. Geophys. Space Phys., 18:753-770.

van Hinte, J. E., 1976. A Cretaceous time scale. Bull. Am. Assoc. Pet. Geol., 60:498-516.

Date of Initial Receipt: February 14, 1983 Date of Acceptance: June 22, 1983 
Table 4. Paleomagnetic results, Site 550.

\begin{tabular}{|c|c|c|c|c|c|c|c|}
\hline & Sub-bottom & & $\begin{array}{r}\text { Before } \\
\text { magnetiz }\end{array}$ & & Demagnetization & & $\begin{array}{r}\text { Aft } \\
\text { demagne }\end{array}$ \\
\hline $\begin{array}{l}\text { Core-Sample } \\
\text { (interval in } \mathrm{cm} \text { ) }\end{array}$ & $\begin{array}{l}\text { depth } \\
\text { (m) }\end{array}$ & $\begin{array}{l}\text { D } \\
\left({ }^{\circ}\right)\end{array}$ & $\begin{array}{l}1 \\
\left({ }^{\circ}\right)\end{array}$ & $\begin{array}{l}\text { Int } \\
(\mu G)\end{array}$ & $\begin{array}{l}\text { field } \\
(\mathrm{Oe})\end{array}$ & $\begin{array}{l}\text { D } \\
\left({ }^{\circ}\right)\end{array}$ & $\begin{array}{c}1 \\
\left({ }^{\circ}\right)\end{array}$ \\
\hline Hole 550 & & & & & & & \\
\hline $5-1,26-28$ & 128.26 & 136.8 & 11.8 & 0.158 & 25 & 155.5 & 62.7 \\
\hline $5-1,124-127$ & 129.24 & 28.5 & -45.2 & 0.035 & NRM & & \\
\hline $5-2,26-28$ & 129.76 & 342.4 & -19.0 & 36.836 & 50 & 340.0 & -19.1 \\
\hline $5-2,111-113$ & 130.61 & 305.8 & 07.3 & 0.713 & 50 & 300.1 & -11.6 \\
\hline $5-3,23-29$ & 131.27 & 5.0 & 1.9 & 0.004 & NRM & & \\
\hline $5-3,112-114$ & 132.12 & 198.4 & 58.3 & 1.975 & 50 & 184.4 & 68.4 \\
\hline $5-4,27-29$ & 132.77 & 259.2 & -67.0 & 0.066 & NRM & & \\
\hline $5-4,113-115$ & 133.63 & 82.8 & 19.2 & 0.108 & NRM & & \\
\hline $5-5,31-33$ & 134.31 & 175.0 & -9.8 & 0.093 & NRM & & \\
\hline $5-6,33-35$ & 135.83 & 162.4 & -0.3 & 0.071 & NRM & & \\
\hline $5-7,31-33$ & 137.31 & 340.1 & -33.6 & 0.085 & NRM & & \\
\hline $6-1,31-33$ & 137.81 & 82.2 & -21.0 & 1.126 & 50 & 62.0 & -19.5 \\
\hline $6-2,35-37$ & 139.35 & 145.1 & 42.0 & 0.121 & 25 & 222.4 & -79.5 \\
\hline $6-3,139-141$ & 141.89 & 240.4 & -42.3 & 0.548 & 25 & 213.9 & -35.1 \\
\hline $6-4,34-36$ & 142.34 & 113.4 & -15.3 & 0.198 & 25 & 146.3 & 25.6 \\
\hline $6-5,11-13$ & 143.61 & 2.9 & 34.1 & 0.093 & NRM & & \\
\hline $6-6,129-131$ & 146.29 & 281.4 & 62.2 & 0.113 & 25 & 267.5 & 70.9 \\
\hline $6-7,37-39$ & 146.87 & 12.9 & -1.9 & 0.315 & 25 & 259.9 & 60.3 \\
\hline $7-1,29-31$ & 147.29 & 82.9 & 43.8 & 4.337 & 50 & 80.0 & 43.0 \\
\hline $7-3,124-126$ & 151.24 & 302.9 & -49.0 & 0.096 & NRM & & \\
\hline $7-4,18-20$ & 151.68 & 323.4 & -47.0 & 2.321 & 50 & 329.7 & -49.0 \\
\hline $7-5,126-128$ & 154.26 & 140.8 & 7.5 & 0.124 & 25 & 61.9 & -48.1 \\
\hline $7-6,26-28$ & 154.76 & 50.0 & 55.9 & 0.114 & NRM & & \\
\hline $8-3,124-126$ & 160.74 & 349.3 & 9.9 & 0.041 & NRM & & \\
\hline $8-4,56-58$ & 161.56 & 136.6 & -18.9 & 0.221 & 25 & 87.6 & -74.3 \\
\hline $8-5,21-23$ & 162.71 & 147.2 & -9.0 & 0.092 & NRM & & \\
\hline $9-1,73-75$ & 166.73 & 178.2 & 36.7 & 0.140 & NRM & & \\
\hline $9-3,18-20$ & 169.18 & 11.8 & 2.2 & 0.381 & 25 & 14.6 & -16.6 \\
\hline $9-4,67-69$ & 171.17 & 137.3 & 19.3 & 0.101 & NRM & & \\
\hline $9-5,7-9$ & 172.07 & 59.8 & 56.4 & 0.129 & NRM & & \\
\hline $9-6,16-18$ & 173.66 & 104.5 & 28.3 & 0.081 & NRM & & \\
\hline $9-6,127-129$ & 174.77 & 275.9 & 21.9 & 0.032 & NRM & & \\
\hline $10-1,134-136$ & 176.84 & 21.5 & -34.3 & 0.103 & NRM & & \\
\hline $10-2,111-113$ & 178.11 & 238.5 & -44.6 & 0.120 & NRM & & \\
\hline $10-3,41-43$ & 178.91 & 23.5 & 34.6 & 0.453 & 25 & 40.2 & 26.3 \\
\hline $11-1,23-25$ & 185.23 & 84.2 & -46.8 & 0.168 & 25 & 104.2 & -13.6 \\
\hline $11-2,53-55$ & 187.03 & 83.2 & -22.3 & 0.178 & 25 & 127.9 & -56.2 \\
\hline $11-3,99-101$ & 188.99 & 339.9 & -48.6 & 0.840 & 25 & 309.3 & -61.4 \\
\hline $12-3,37-39$ & 197.87 & 249.3 & -43.0 & 1.773 & 50 & 247.6 & -27.1 \\
\hline $12-4,25-27$ & 199.25 & 205.7 & 8.5 & 0.163 & 25 & 191.2 & -14.8 \\
\hline $13-1,24-26$ & 204.22 & 111.7 & -38.9 & 0.112 & NRM & & \\
\hline $14-1,37-39$ & 213.87 & 53.0 & -54.0 & 0.091 & NRM & & \\
\hline $14-2,43-45$ & 215.43 & 80.6 & 54.6 & 0.180 & 25 & 335.5 & -16.7 \\
\hline $14-3,31-33$ & 216.81 & 330.0 & -57.6 & 0.236 & 25 & 16.8 & -13.6 \\
\hline $14-3,106-108$ & 217.56 & 231.0 & -58.0 & 0.024 & NRM & & \\
\hline $15-1,27-29$ & 223.27 & 298.3 & 7.3 & 0.047 & NRM & & \\
\hline $15-1,77-79$ & 223.37 & 347.0 & 2.1 & 0.009 & NRM & & \\
\hline $16-1,19-21$ & 232.69 & 348.9 & 6.8 & 0.153 & 25 & 32.3 & -13.3 \\
\hline $16-2,18-20$ & 234.18 & 106.3 & 4.8 & 0.203 & 25 & 94.0 & -8.7 \\
\hline $16-2,91-93$ & 234.91 & 38.8 & 46.8 & 0.060 & NRM & & \\
\hline $16-3,23-25$ & 235.73 & 342.1 & -76.4 & 0.280 & 25 & 140.4 & 62.9 \\
\hline $16-3,95-97$ & 236.45 & 0.0 & 16.8 & 1.948 & 50 & 30.5 & 18.8 \\
\hline $16-4,14-16$ & 237.14 & 35.3 & 13.9 & 16.433 & 50 & 35.3 & 9.2 \\
\hline $17-1,131-133$ & 243.31 & 19.0 & 38.7 & 2.987 & 50 & 8.3 & 43.8 \\
\hline $17-2,20-22$ & 243.70 & 256.7 & 15.1 & 4.000 & 50 & 253.0 & 22.3 \\
\hline $17-3,28-30$ & 245.28 & 92.7 & 18.3 & 4.308 & 50 & 100.0 & 18.8 \\
\hline $18-1,43-45$ & 251.93 & 20.6 & -27.1 & 0.410 & 25 & 12.3 & -22.1 \\
\hline $18-2,25-27$ & 253.25 & 13.8 & -47.4 & 0.159 & NRM & & \\
\hline $18-3,24-26$ & 254.74 & 108.4 & -15.3 & 0.122 & NRM & & \\
\hline $18-4,8-10$ & 256.08 & 18.6 & -21.1 & 0.103 & NRM & & \\
\hline $19-1,29-31$ & 261.29 & 340.4 & -11.9 & 0.152 & 25 & 163.0 & -31.6 \\
\hline $19-1,96-98$ & 261.96 & 306.0 & -36.9 & 0.160 & NRM & & \\
\hline $19-2,17-20$ & 262.67 & 6.2 & 7.0 & 0.430 & 25 & 22.1 & -2.7 \\
\hline $19-3,5-8$ & 264.05 & 17.2 & -25.5 & 0.155 & NRM & & \\
\hline $20-11,6-8$ & 279.80 & 96.1 & -2.8 & 13.797 & 50 & 100.9 & -1.9 \\
\hline $21-1,19-21$ & 280.19 & 101.3 & 25.9 & 0.027 & NRM & & \\
\hline $21-2,40-42$ & 281.90 & & & & & & \\
\hline $21-2,108-110$ & 282.58 & 257.8 & -32.8 & 0.180 & NRM & & \\
\hline $21-3,28-30$ & 283.28 & 29.2 & -24.5 & 0.017 & NRM & & \\
\hline $22-1,58-60$ & 290.08 & 252.7 & -61.2 & 1.743 & 50 & 248.6 & -56.7 \\
\hline $22-1,131-133$ & 290.81 & 121.9 & 63.8 & 4.460 & 200 & 155.7 & -44.1 \\
\hline $22-2,51-53$ & 291.51 & 147.9 & 74.1 & 5.468 & 50 & 75.0 & 77.0 \\
\hline $22-2,113-115$ & 292.13 & 185.0 & 66.0 & 3.347 & 50 & 177.0 & 40.5 \\
\hline $22-3,58-60$ & 293.08 & 242.4 & 77.7 & 12.300 & 50 & 355.7 & 54.7 \\
\hline $22-4,58-60$ & 294.58 & 191.4 & 80.1 & 14.949 & 50 & 192.6 & 71.1 \\
\hline $22-5,28-30$ & 295.78 & 23.5 & 77.5 & 3.389 & 50 & 195.8 & 76.8 \\
\hline $23-1,118-120$ & 200.18 & 48.8 & -44.8 & 0.869 & 25 & 43.3 & -47.4 \\
\hline $23-2,83-85$ & 301.33 & 255.9 & 62.4 & 10.684 & 50 & 253.1 & 63.5 \\
\hline $23-3,77-79$ & 302.77 & 120.0 & 53.4 & 21.867 & 50 & 120.0 & 41.8 \\
\hline $23-4,123-125$ & 304.73 & 332.9 & 87.2 & 2.373 & 50 & 40.4 & -49.6 \\
\hline $23-5,84-86$ & 305.84 & 125.1 & 67.9 & 17.333 & 50 & 139.3 & 68.1 \\
\hline $23-11,17-19$ & 308.17 & 222.4 & -79.0 & 0.742 & 50 & 345.7 & -78.8 \\
\hline $24-1,5-6$ & 308.55 & 260.1 & 57.4 & 28.710 & 50 & 249.9 & 53.0 \\
\hline $24-1,106-108$ & 309.56 & 311.6 & 41,1 & 28.189 & 200 & 316.3 & 29.7 \\
\hline $24-2,4-6$ & 310.04 & 48.5 & 72.1 & 18.176 & 75 & 273.9 & -48.0 \\
\hline $24-2,136-138$ & 311.36 & 69.3 & 62.1 & 20.634 & 200 & 187.5 & -5.8 \\
\hline $24-3,29-31$ & 311.79 & 75.7 & 59.6 & 20.783 & 150 & 137.4 & 53.0 \\
\hline $24-3,123-125$ & 312.73 & 346.6 & 45.4 & 27.164 & 150 & 8.5 & -24.7 \\
\hline
\end{tabular}


Table 4. (Continued).

\begin{tabular}{|c|c|c|c|c|c|c|c|}
\hline & Sub-bottom & & $\begin{array}{r}\text { Befo } \\
\text { emagnet: }\end{array}$ & & & & $\begin{array}{r}\text { A } \\
\text { demagn }\end{array}$ \\
\hline $\begin{array}{l}\text { Core-Sample } \\
\text { (interval in } \mathrm{cm} \text { ) }\end{array}$ & $\begin{array}{l}\text { depth } \\
\text { (m) }\end{array}$ & $\underset{\left({ }^{\circ}\right)}{\mathrm{D}}$ & $\begin{array}{l}1 \\
\left({ }^{\circ}\right)\end{array}$ & $\begin{array}{l}\text { Int } \\
(\mu \mathrm{G})\end{array}$ & $\begin{array}{l}\text { field } \\
(\mathrm{Oe})\end{array}$ & $\underset{\left({ }^{\circ}\right)}{\mathrm{D}}$ & $\begin{array}{l}\text { I } \\
\left({ }^{\circ}\right)\end{array}$ \\
\hline Hole 550 (Cont.) & & & & & & & \\
\hline $24-4,66-68$ & 313.66 & 333.2 & 71.2 & 12.634 & 75 & 61.3 & -31.1 \\
\hline $24-4,121-123$ & 314.21 & 339.9 & 32.4 & 15.287 & 150 & 194.9 & -37.0 \\
\hline $24-5,45-47$ & 314.95 & 326.7 & 57.4 & 21.135 & 100 & 210.6 & -49.6 \\
\hline $24-5,101-103$ & 315.51 & 317.9 & 54.2 & 26.673 & 150 & 284.9 & 48.6 \\
\hline $25-1,69-71$ & 318.69 & 97.3 & 56.8 & 29.252 & 200 & 128.1 & 37.1 \\
\hline $25-1,128-130$ & 319.28 & 38.0 & 39.2 & 11.872 & 150 & 44.6 & -45.4 \\
\hline $25-2,6-8$ & 319.56 & 28.8 & 59.6 & 18.703 & 100 & 0.7 & -38.2 \\
\hline $25-3,84-86$ & 321.84 & 296.7 & 50.4 & 15.785 & 100 & 46.7 & -0.5 \\
\hline $25-4,16-18$ & 433.66 & 135.1 & 78.1 & 17.366 & 100 & 157.3 & -1.5 \\
\hline $25-5,15-17$ & 324.15 & 37.4 & 50.0 & 13.666 & 100 & 7.8 & -22.5 \\
\hline $26-1,17-19$ & 327.67 & 304.9 & 41.7 & 42.004 & 400 & 259.4 & 46.3 \\
\hline $26-1,124-126$ & 328.74 & 326.3 & 37.8 & 38.100 & 150 & 329.0 & 37.1 \\
\hline $27-1,33-36$ & 337.33 & 255.3 & -0.2 & 29.273 & 150 & 243.5 & -36.6 \\
\hline $27 \cdot 2,28-31$ & 338.78 & 348.5 & -29.6 & 32.426 & 150 & 347.9 & -36.7 \\
\hline $27-3,23-26$ & 340.23 & 51.7 & 9.1 & 32.547 & 150 & 57.5 & -32.7 \\
\hline $27-3,146-149$ & 341.46 & 296.0 & -25.4 & 23.348 & 150 & 277.8 & -33.6 \\
\hline $27-4,117-120$ & 342.67 & 143.8 & 50.9 & 58.304 & 150 & 150.6 & 40.4 \\
\hline $27-5,20-23$ & 343.20 & 295.4 & 48.2 & 31.351 & 150 & 42.0 & 0.3 \\
\hline $27-6,12-15$ & 344.6 & 311.8 & 39.8 & 29.348 & 150 & 311.4 & 34.9 \\
\hline $27-6,102-105$ & 345.02 & 45.8 & -50.5 & 32.826 & 150 & 45.6 & -54.5 \\
\hline $27-7,16-18$ & 346.16 & 229.1 & -29.2 & 22.234 & 150 & 222.8 & -33.9 \\
\hline $28-1,44-47$ & 346.94 & 345.6 & 29.3 & 33.882 & 150 & 345.8 & 26.6 \\
\hline $28-1,112-114$ & 347.62 & 298.7 & -20.4 & 25.41 & 150 & 297.6 & -22.1 \\
\hline $28-2,26-29$ & 348.26 & 290.3 & -2.0 & 12.312 & 150 & 288.6 & -7.3 \\
\hline $28-2,138-140$ & 349.38 & 68.1 & -12.1 & 19.236 & 150 & 72.9 & -14.1 \\
\hline $28-3,36-38$ & 349.86 & 318.1 & 52.6 & 18.716 & 150 & 321.2 & 46.8 \\
\hline $28-4,32-34$ & 351.32 & 183.5 & 48.7 & 21.840 & 150 & 182.1 & 49.4 \\
\hline $28-5,24-26$ & 352.34 & 136.2 & 56.0 & 29.927 & 150 & 139.4 & 53.4 \\
\hline $29-1,15-18$ & 356.15 & 97.2 & 49.5 & 37.912 & 150 & 96.7 & 43.0 \\
\hline $29-2,26-29$ & 357.76 & 268.5 & 41.7 & 46.888 & 150 & 270.2 & 37.6 \\
\hline $29-3,27-30$ & 359.27 & 107.3 & 56.9 & 35.857 & 150 & 47.5 & 19.9 \\
\hline $29-3,103-105$ & 360.03 & 174.0 & -9.8 & 19.240 & $i 50$ & 196.8 & -30.1 \\
\hline $29-4,29-32$ & 360.79 & 149.4 & 20.4 & 13.074 & 150 & 141.9 & -8.9 \\
\hline $29-5,17-20$ & 362.17 & 341.4 & 12.7 & 12.820 & 150 & 335.6 & -32.9 \\
\hline $29-6,38-41$ & 363.88 & 137.7 & 28.2 & 12.221 & 150 & 149.4 & -25.0 \\
\hline $30-1,11-14$ & 365.61 & 36.2 & 2.6 & 18.888 & 150 & 357.8 & -61.0 \\
\hline $30-1,88-91$ & 366.38 & 134.3 & -37.3 & 9.877 & 150 & 138.6 & -27.6 \\
\hline $30-2,2-5$ & 367.02 & 58.9 & 61.6 & 10.072 & 150 & 18.2 & 53.3 \\
\hline $30-3,32-35$ & 368.82 & 103.7 & -2.1 & 0.350 & 50 & 168.0 & -31.8 \\
\hline $30-4,10-13$ & 370.10 & 337.8 & 82.5 & 3.867 & 50 & 194.7 & -9.4 \\
\hline $30-5,14-17$ & 371.64 & 217.2 & 21.7 & 8.740 & 50 & 221.7 & -24.0 \\
\hline $30-6,14-17$ & 373.14 & 246.1 & -3.2 & 8.306 & 150 & 249.9 & -39.6 \\
\hline $31-1,18-21$ & 375.18 & 63.5 & -14.9 & 6.161 & 150 & 59.9 & -47.9 \\
\hline $31-2,29-31$ & 376.87 & 118.6 & 49.1 & 13.327 & 150 & 85.3 & -17.9 \\
\hline $31-3,29-32$ & 378.29 & 135.1 & 10.7 & 20.395 & 150 & 149.3 & -24.4 \\
\hline $31-4,32-34$ & 379.82 & 273.3 & 5.1 & 21.749 & 150 & 264.3 & -13.4 \\
\hline $31-5,26-28$ & 381.26 & 90.8 & -0.7 & 0.438 & 50 & 68.5 & -64.4 \\
\hline $32-1,14-16$ & 384.64 & 29.5 & 65.1 & 9.430 & 50 & 111.4 & 36.7 \\
\hline $32-2,31-33$ & 386.31 & 321.5 & -22.4 & 6.914 & 150 & 307.7 & -37.3 \\
\hline $32-3,24-26$ & 387.74 & 207.6 & 8.8 & 17.064 & 150 & 202.8 & -32.6 \\
\hline $32-4,27-29$ & 389.27 & 58.4 & 28.0 & 4.181 & 150 & 69.9 & -39.4 \\
\hline $32-5,21-23$ & 390.71 & 121.1 & 17.4 & 13.186 & 150 & 123.3 & -27.4 \\
\hline $32-6,45-47$ & 392.45 & 4.1 & -4.4 & 21.919 & 150 & 0.1 & -34.8 \\
\hline $32-7,37-39$ & 393.87 & 42.1 & 23.1 & 13.894 & 150 & 53.8 & -20.3 \\
\hline $33-1,16-18$ & 394.16 & 151.1 & 30.8 & 2.717 & 150 & 161.6 & -36.6 \\
\hline $33-2,23-25$ & 395.73 & 39.9 & 55.4 & 11.332 & 150 & 43.3 & -36.6 \\
\hline $33-2,129-131$ & 396.79 & 342.5 & -0.0 & 10.909 & 150 & 352.1 & -29.3 \\
\hline $33-3,19-21$ & 397.19 & 93.2 & 58.0 & 18.371 & 150 & 98.1 & -22.3 \\
\hline $33-4,24-26$ & 398.74 & 99.3 & 88.8 & 18.682 & 150 & 269.5 & -26.0 \\
\hline $33-4,98-100$ & 399.48 & 103.7 & 46.7 & 63.021 & 150 & 98.4 & 37.4 \\
\hline $33-5,30-32$ & 400.30 & 18.5 & 86.8 & 20.698 & 150 & 135.7 & -19.1 \\
\hline $34-1,131-133$ & 404.81 & 271.2 & 6.5 & 6.726 & 150 & 285.1 & -15.5 \\
\hline $34-2,135-137$ & 406.35 & 250.9 & -3.5 & 0.898 & 150 & 254.5 & -19.9 \\
\hline $34-3,34-36$ & 406.84 & 226.8 & -25.7 & 1.254 & 150 & 227.8 & -42.6 \\
\hline $34-4,16-18$ & 408.16 & 181.2 & -1.2 & 0.073 & NRM & & \\
\hline $34-4,28-30$ & 409.78 & 141.8 & -14.6 & 0.100 & NRM & & \\
\hline $34-6,13-16$ & 411.13 & 320.4 & -9.5 & 23.789 & 150 & 252.2 & -35.3 \\
\hline $34-6,136-138$ & 412.36 & 259.2 & 63.5 & 0.023 & NRM & & \\
\hline $35-1,31-33$ & 413.31 & 317.6 & -35.8 & 0.440 & 150 & 324.6 & -31.5 \\
\hline $35-1,97-99$ & 413.97 & 261.9 & -8.9 & 0.237 & 50 & 26.2 & 6.4 \\
\hline $35-4,83-85$ & 418.33 & 291.0 & -25.4 & 0.054 & NRM & & \\
\hline $35-5,51-53$ & 419.51 & 1.3 & -17.7 & 0.876 & 50 & & \\
\hline $36-2,101-103$ & 425.5 & 110.4 & 57.9 & 69.120 & 50 & 99.9 & 55.7 \\
\hline $36-3,146-148$ & 426.96 & 41.1 & 84.6 & 3.969 & so & 168.8 & 42.5 \\
\hline $36-4,124-126$ & 428.24 & 138.4 & 28.2 & 0.047 & NRM & & \\
\hline $37-1,10-12$ & 432.10 & 210.7 & 57.7 & 0.261 & 25 & 211.2 & 70.3 \\
\hline $27-2,54-56$ & 434.04 & 321.7 & 83.1 & 0.235 & 25 & 20.5 & 72.2 \\
\hline $37-3,42-44$ & 435.42 & 126.1 & -.56 & 0.048 & NRM & & \\
\hline $37-3,119-121$ & 436.19 & 7.6 & 9.8 & 0.028 & NRM & & \\
\hline $37-4,125-127$ & 437.75 & 327.6 & 29.5 & 0.039 & NRM & & \\
\hline $37-5,52-54$ & 438.52 & 102.5 & -9.5 & 0.017 & NRM & & \\
\hline $38-1,127-130$ & 442.77 & 161.4 & -43.3 & 0.490 & 25 & 187.8 & -47.8 \\
\hline $38-2,28-30$ & 443.28 & 305.1 & 46.0 & 0.069 & NRM & & \\
\hline $38-3,10-12$ & 444.60 & 41.3 & 42.1 & 0.012 & NRM & & \\
\hline $38-5,33-35$ & 447.83 & 181.7 & 54.0 & 0.105 & NRM & & \\
\hline $38-5,86-88$ & 448.36 & 345.1 & 35.6 & 10.249 & 150 & 314.0 & 38.7 \\
\hline $38-6,9-11$ & 449.09 & 285.3 & -4.8 & 0.161 & 25 & 273.7 & -56.3 \\
\hline $38-6,191-93$ & 449.91 & 317.5 & -22.3 & 0.429 & 50 & 329.3 & -27.2 \\
\hline
\end{tabular}


Table 4. (Continued).

\begin{tabular}{|c|c|c|c|c|c|c|c|c|}
\hline & & & $\begin{array}{l}\text { Before } \\
\text { nagnetiz }\end{array}$ & & Demagnetization & & $\begin{array}{r}\text { Aft } \\
\text { demagne }\end{array}$ & $\begin{array}{l}\text { ter } \\
\text { etization }\end{array}$ \\
\hline $\begin{array}{l}\text { Core-Sample } \\
\text { (interval in } \mathrm{cm} \text { ) }\end{array}$ & $\begin{array}{l}\text { depth } \\
\text { (m) }\end{array}$ & $\begin{array}{l}D \\
\left({ }^{\circ}\right)\end{array}$ & $\begin{array}{l}1 \\
\left({ }^{\circ}\right)\end{array}$ & $\begin{array}{l}\text { Int } \\
(\mu \mathrm{G})\end{array}$ & $\begin{array}{l}\text { field } \\
\text { (Oe) }\end{array}$ & $\begin{array}{l}\mathrm{D} \\
\left({ }^{\circ}\right)\end{array}$ & $\begin{array}{l}1 \\
\left({ }^{\circ}\right)\end{array}$ & $\begin{array}{l}\text { Int } \\
(\mu \mathrm{G})\end{array}$ \\
\hline Hole 550 (Cont.) & & & & & & & & \\
\hline $39-1,11-14$ & 451.11 & 140.2 & 86.2 & 4.261 & 25 & 148.7 & 83.7 & 4.095 \\
\hline $39-1,137-140$ & 452.37 & 299.1 & -28.9 & 3.295 & 100 & 285.4 & -35.9 & 3.075 \\
\hline $39-8,29-31$ & 452.34 & 91.9 & -30.1 & 0.074 & NRM & & & \\
\hline $39-2,109-111$ & 453.59 & 271.6 & -27.4 & 7.931 & 100 & 274.6 & -31.6 & 8.166 \\
\hline $39-3,134-137$ & 455.34 & 248.8 & -36.7 & 0.038 & NRM & 2100 & Novo & . \\
\hline $39-4,6-9$ & 455.56 & 24.9 & -24.4 & 4.668 & 25 & 27.7 & -22.6 & 4.550 \\
\hline $39-5,50-53$ & 457.50 & 20.6 & 60.6 & 29.347 & 150 & 203.7 & -26.3 & 4.622 \\
\hline $39-5,139-142$ & 458.39 & 299.5 & 71.3 & 0.153 & 25 & 343.7 & 64.2 & 0.166 \\
\hline $41-2,37-39$ & 471.87 & 114.0 & 80.4 & 6.613 & 25 & 100.6 & 84.3 & 6.533 \\
\hline $41-2,128-131$ & 472.78 & 332.2 & -74.8 & 78.494 & 150 & 88.2 & 40.3 & 0.869 \\
\hline $41-3,26-29$ & 473.26 & 112.1 & 80.4 & 21.80 & 25 & 127.8 & 77.7 & 17.471 \\
\hline $42-1,106-108$ & 480.5 & 9.8 & 61.7 & 3.190 & 25 & 12.8 & 59.6 & 3.422 \\
\hline $42-2,45-48$ & 481.45 & 87.4 & 63.3 & 0.739 & 25 & 88.2 & 66.9 & 0.878 \\
\hline $43-1,36-38$ & 489.36 & 340.0 & 71.6 & 10.986 & 25 & 336.9 & 73.1 & 10.453 \\
\hline $43-2,26-28$ & 490.76 & 131.8 & 74.0 & 6.728 & 50 & 133.6 & 78.1 & 6.618 \\
\hline $43-3,8-10$ & 492.08 & 332.4 & 62.9 & 11.683 & 50 & 328.9 & 63.0 & 11.405 \\
\hline $47-1,20-22$ & 522.70 & 294.5 & 67.0 & 30.861 & 50 & 297.2 & 74.7 & 15.132 \\
\hline $47-1,118-120$ & 523.18 & 2.2 & 29.7 & 5.681 & 100 & 77.1 & -42.6 & 4.223 \\
\hline $47-2,28-30$ & 524.28 & 15.6 & 74.5 & 13.613 & 150 & 184.3 & -52.1 & 2.737 \\
\hline $47-2,117-121$ & 524.69 & 306.1 & 73.8 & 10.960 & 150 & 319.2 & -24.9 & 0.984 \\
\hline $47-3,51-53$ & 526.01 & 254.6 & 67.0 & 30.721 & 150 & 269.2 & 58.1 & 2.376 \\
\hline $47-3,105-107$ & 526.05 & 162.4 & 67.5 & 12.317 & 150 & 188.4 & 54.8 & 2.025 \\
\hline Hole 550B & & & & & & & & \\
\hline $1-1,52-54$ & 456.52 & 298.7 & 60.2 & 22.893 & 150 & 303.4 & 64.7 & 4.460 \\
\hline $1-2,95-97$ & 458.45 & 137.3 & 57.5 & 40.250 & 150 & 157.4 & 46.0 & 21.375 \\
\hline $1-3,112-114$ & 460.12 & 300.9 & 63.2 & 23.511 & 150 & 297.7 & 68.9 & 5.887 \\
\hline $1-4,128-130$ & 461.78 & 136.7 & 78.1 & 27.905 & 300 & 306.0 & -23.0 & 3.215 \\
\hline $1-5,144-146$ & 463.44 & 36.6 & 57.1 & 92.584 & 500 & 330.6 & -4.7 & 2.493 \\
\hline $1-6,9-11$ & 463.57 & 246.5 & 63.8 & 73.764 & 400 & 285.4 & -4.7 & 0.145 \\
\hline $2-1,55-57$ & 466.05 & 117.4 & 62.8 & 23.865 & 500 & 9.9 & 35.5 & 0.448 \\
\hline $2-1,123-125$ & 466.73 & 5.4 & 50.9 & 20.090 & 150 & 56.0 & 32.0 & 0.734 \\
\hline $2-2,99-101$ & 467.99 & 344.3 & 74.2 & 58.929 & 500 & 359.3 & 19.0 & 4.513 \\
\hline $2-3,17-19$ & 468.67 & 324.4 & 73.8 & 54.678 & 300 & 350.8 & 83.5 & 17.735 \\
\hline $2-3,21-24$ & 468.71 & 356.5 & 88.5 & 25.564 & 200 & 330.1 & -43.9 & 0.062 \\
\hline $2-3,32-34$ & 468.82 & 305.2 & 43.4 & 25.788 & 200 & 37.8 & -59.2 & 1.982 \\
\hline $2-3,81-83$ & 469.31 & 352.7 & 53.3 & 26.096 & 150 & 159.1 & -16.4 & 1.617 \\
\hline $2-3,113-115$ & 469.63 & 68.3 & 80.8 & 25.090 & 150 & 267.1 & -24.9 & 1.458 \\
\hline $3-1,5-6$ & 475.05 & 256.5 & -64.4 & 20.214 & 100 & 3.9 & -81.7 & 2.235 \\
\hline $3-1,137-139$ & 476.37 & 197.0 & 50.7 & 20.843 & 300 & 272.8 & -66.1 & 0.387 \\
\hline $3-2,21-23$ & 478.21 & 128.2 & 69.9 & 17.200 & 300 & 139.7 & 64.2 & 1.882 \\
\hline $4-1,26-29$ & 484.76 & 193.8 & 68.1 & 8.128 & 300 & 199.9 & 68.1 & 6.810 \\
\hline $4-2,22-25$ & 486.22 & 62.6 & 63.1 & 10.433 & 300 & 50.2 & 61.2 & 4.232 \\
\hline $5-1,33-36$ & 494.33 & 15.9 & 57.5 & 14.469 & 300 & 14.8 & 52.5 & 3.715 \\
\hline $5-2,14-17$ & 495.64 & 141.5 & 60.8 & 20.954 & 300 & 124.3 & 63.5 & 3.714 \\
\hline $5-3,10-13$ & 497.10 & 257.7 & 58.7 & 5.597 & 300 & 308.0 & 42.2 & 0.807 \\
\hline $5-4,116-118$ & 499.66 & 136.3 & 64.5 & 6.436 & 300 & 116.2 & 58.9 & 0.455 \\
\hline $7=1,75-77$ & 513.75 & 306.3 & 67.6 & 25.483 & 200 & 333.2 & 72.3 & 4.171 \\
\hline $7-2,101-103$ & 515.51 & 325.2 & 59.5 & 29.017 & 200 & 323.1 & 49.1 & 6.612 \\
\hline $7-3,42-44$ & 518.42 & 119.8 & 65.1 & 34.765 & 200 & 105.9 & 52.4 & 14.195 \\
\hline $8-1,128-130$ & 523.78 & 202.6 & 70.6 & 26.337 & 400 & 347.3 & 62.2 & 2.800 \\
\hline $8-2,9-11$ & 524.00 & 230.3 & 49.3 & 21.742 & 150 & 220.6 & 39.1 & 2.627 \\
\hline $8-2,121-123$ & 525.21 & 255.0 & 84.8 & 18.035 & 200 & 45.4 & -32.2 & 4.677 \\
\hline $8-3,115-117$ & 526.65 & 301.5 & 73.3 & 17.250 & 200 & 117.3 & -53.2 & 0.857 \\
\hline $8-3,123-125$ & 526.73 & 30.9 & -74.1 & 22.563 & NRM & & & \\
\hline $8-3,127-129$ & 526.77 & 54.1 & -66.4 & 23.503 & 200 & 335.4 & -71.7 & 1.097 \\
\hline $8-3,131-133$ & 526.81 & 300.5 & 67.5 & 23.152 & 300 & 106.8 & 44.0 & 0.118 \\
\hline $8-3,147-149$ & 526.97 & 286.0 & 64.2 & 28.129 & 200 & 290.3 & 65.5 & 2.747 \\
\hline $8-4,11-13$ & 527.11 & 307.4 & 66.3 & 25.810 & 200 & 267.6 & 58.8 & 2.823 \\
\hline $8-4,125-127$ & 528.25 & 356.2 & 69.8 & 23.755 & 200 & 17.0 & 60.8 & 1.628 \\
\hline $8-5,93-95$ & 529.43 & 289.8 & 67.6 & 35.543 & 200 & 306.8 & 65.8 & 5.025 \\
\hline $8-6,9-11$ & 530.09 & 9.6 & 54.9 & 30.657 & 200 & 8.4 & 62.0 & 3.133 \\
\hline $9-1,42-45$ & 532.45 & 131.5 & 66.7 & 23.260 & 300 & 162.0 & 31.5 & 0.800 \\
\hline $9-2,44-46$ & 533.94 & 324.9 & 68.5 & 28.177 & 200 & 324.3 & 74.3 & 4.301 \\
\hline $9-3,33-35$ & 535.85 & 31.4 & 24.5 & 0.126 & NRM & & & \\
\hline $9-3,85-87$ & 535.33 & 113.4 & -24.7 & 0.216 & 25 & 78.6 & 10.6 & 0.259 \\
\hline $9-4,30-32$ & 536.80 & 265.4 & -14.8 & 0.175 & 25 & 236.1 & -28.5 & 0.478 \\
\hline $9-5,22-24$ & 538.22 & 81.1 & 58.7 & 0.311 & 25 & 145.8 & 3.9 & 0.066 \\
\hline $10-1,55-57$ & 542.07 & 343.6 & 49.4 & 18.222 & 150 & 252.1 & -19.5 & 3.419 \\
\hline $10-3,14-16$ & 544.64 & 131.8 & 59.4 & 40.185 & 300 & 128.1 & 52.2 & 9.561 \\
\hline $10-4,78-80$ & 546.78 & 18.2 & 41.4 & 0.478 & 25 & 5.4 & 3.7 & 0.221 \\
\hline $10-4,118-120$ & 547.18 & 333.6 & 59.0 & 0.242 & 50 & 336.4 & 50.0 & 0.072 \\
\hline $10-5,67-69$ & 548.17 & 352.6 & -13.6 & 0.253 & 25 & 352.6 & -27.2 & 0.228 \\
\hline $11-1,35-37$ & 551.35 & 325.6 & 66.5 & 27.781 & 150 & 21.4 & 57.4 & 2.652 \\
\hline $11-2,25-27$ & 552.75 & 26.6 & 74.4 & 1.320 & 50 & 185.6 & 69.0 & 0.460 \\
\hline $11-3,107-109$ & 555.07 & 295.7 & 36.7 & 0.139 & 25 & 301.8 & 78.1 & 0.350 \\
\hline $11-4,28-30$ & 555.78 & 71.1 & 17.4 & 0.303 & 25 & 14.3 & -23.8 & 0.075 \\
\hline $12-5,32-34$ & 560.82 & 202.9 & 61.1 & 0.355 & 50 & 74.4 & 82.4 & 0.042 \\
\hline $12-2,130-132$ & 563.30 & 295.9 & 60.0 & 29.548 & 300 & 280.3 & 42.1 & 26.343 \\
\hline $12-3,9-11$ & 563.59 & 51.3 & 49.5 & 29.756 & 400 & 42.4 & 33.5 & 1.184 \\
\hline $12-4,58-60$ & 565.58 & 216.2 & 78.8 & 17.440 & 200 & 265.3 & 73.2 & 1.256 \\
\hline $13-1,19-22$ & 570.19 & 11.9 & 51.7 & 21.997 & 300 & 11.4 & 14.9 & 3.867 \\
\hline $13-2,38-40$ & 571.88 & 221.4 & 57.0 & 83.854 & 200 & 207.8 & 35.9 & 21.92 \\
\hline $13-3,15-17$ & 573.15 & 68.9 & 54.2 & 36.158 & 400 & 79.9 & 33.2 & 4.616 \\
\hline $13-4,99-101$ & 575.49 & 88.0 & 61.9 & 0.697 & 25 & 100.3 & 68.3 & 0.775 \\
\hline $13-5,65-67$ & 576.65 & 211.9 & 32.0 & 0.315 & 25 & 215.1 & 60.6 & 0.342 \\
\hline $13-6,63-66$ & 575.13 & 158.7 & 40.1 & 0.485 & 25 & 103.0 & 70.0 & 0.194 \\
\hline $13-7,15-17$ & 579.15 & 3.0 & 5.8 & 2.270 & 50 & 27.7 & 21.2 & 0.828 \\
\hline
\end{tabular}


Table 4. (Continued).

\begin{tabular}{|c|c|c|c|c|c|c|c|c|c|}
\hline \multirow[b]{2}{*}{$\begin{array}{l}\text { Core-Sample } \\
\text { (interval in } \mathrm{cm} \text { ) }\end{array}$} & \multirow{2}{*}{$\begin{array}{l}\text { Sub-bottom } \\
\text { depth } \\
\text { (m) }\end{array}$} & \multicolumn{3}{|c|}{$\begin{array}{c}\text { Before } \\
\text { demagnetization }\end{array}$} & \multirow{2}{*}{$\begin{array}{l}\text { Demagnetization } \\
\text { field } \\
\text { (Oe) }\end{array}$} & \multicolumn{4}{|c|}{$\begin{array}{c}\text { After } \\
\text { demagnetization }\end{array}$} \\
\hline & & $\begin{array}{l}\text { D } \\
\left({ }^{\circ}\right)\end{array}$ & $\begin{array}{c}1 \\
\left({ }^{\circ}\right)\end{array}$ & $\begin{array}{c}\text { Int } \\
(\mu \mathrm{G})\end{array}$ & & $\begin{array}{l}\text { D } \\
\left({ }^{\circ}\right)\end{array}$ & $\begin{array}{l}1 \\
\left({ }^{\circ}\right)\end{array}$ & $\begin{array}{l}\text { Int } \\
(\mu \mathrm{G})\end{array}$ & Polarity \\
\hline \multicolumn{10}{|l|}{ Hole 550B (Cont.) } \\
\hline $14-1,6-8$ & 579.56 & 195.5 & 43.1 & 0.602 & 50 & 194.1 & 42.0 & 0.813 & $\mathrm{~N}$ \\
\hline $14-2,6-8$ & 581.06 & 314.4 & 36.6 & 0.666 & 50 & 301.1 & 32.1 & 0.834 & $\mathrm{~N}$ \\
\hline $14-3,12-14$ & 582.62 & 212.6 & 42.7 & 0.545 & 50 & 198.0 & 49.2 & 0.739 & $\mathrm{~N}$ \\
\hline $14-4,16-18$ & 584.16 & 48.1 & 18.3 & 1.146 & 150 & 47,7 & 9.3 & 0.853 & $\mathbf{R}$ \\
\hline $14-4,126-128$ & 585.26 & 45.6 & -14.1 & 0.168 & NRM & & & & $\mathbf{R}$ \\
\hline $14-5,41-43$ & 585.91 & 331.4 & -18.7 & 0.273 & 100 & 275.0 & -21.7 & 0.350 & $\mathbf{R}$ \\
\hline $14-5,128-130$ & 586.78 & 358.5 & 47.2 & 0.119 & NRM & & & & $\mathrm{N}$ \\
\hline $15-1,51-53$ & 589.51 & 120.6 & 14.0 & 0.228 & 100 & 146.5 & 36.1 & 0.126 & $\mathrm{~N}$ \\
\hline $15-1,133-136$ & 590.33 & 164.7 & 13.1 & 0.073 & NRM & & & & $\mathrm{N}$ \\
\hline $15-2,104-107$ & 591.54 & 90.9 & 47.3 & 0.799 & 50 & 89.1 & 51.0 & 0.880 & $\mathrm{~N}$ \\
\hline $15-3,16-19$ & 592.16 & 315.6 & 38.2 & 0.597 & 50 & 261.8 & 34.6 & 0.571 & $\mathrm{~N}$ \\
\hline $15-4,119-122$ & 594.69 & 173.3 & 40.4 & 0.581 & so & 176.4 & 40.5 & 0.387 & $\mathrm{~N}$ \\
\hline $15-5,9-12$ & 595.09 & 269.1 & 23.8 & 0.421 & 50 & 242.0 & 30.5 & 0.265 & $\mathrm{~N}$ \\
\hline $15-6,21-24$ & 596.71 & 164.2 & 53.1 & 0.306 & 50 & 203.7 & 32.0 & 0.296 & $\mathrm{~N}$ \\
\hline $16-1,14-16$ & 598.64 & 121.5 & -24.5 & 0.253 & 50 & 14.3 & 13.7 & 0.439 & $\mathrm{~N}$ \\
\hline $16-2,72-74$ & 599.72 & 92.6 & 15.3 & 0.347 & 50 & 89.0 & 30.1 & 0.405 & $\mathrm{~N}$ \\
\hline $16-3,8-10$ & 601.58 & 298.7 & 38.0 & 0.349 & 50 & 344.3 & 12.2 & 0.363 & $\mathrm{~N}$ \\
\hline $17-1,11-13$ & 608.11 & 225.6 & 25.2 & 0.163 & 25 & 198.1 & 49.8 & 0.204 & $\mathrm{~N}$ \\
\hline $17-1,116-118$ & 609.16 & 205.1 & 58.5 & 0.149 & NRM & & & & $\mathrm{N}$ \\
\hline $17-2,35-37$ & 609.85 & 142.4 & 35.0 & 0.194 & 25 & 75.5 & -6.5 & 0.315 & $\mathbf{R}$ \\
\hline $17-2,112-114$ & 610.62 & 320.1 & 55.0 & 0.113 & NRM & & & & $\mathrm{N}$ \\
\hline $17-3,26-28$ & 611.26 & 157.6 & 25.5 & 0.320 & 25 & 182.9 & 51.3 & 0.287 & $\mathrm{~N}$ \\
\hline $17-4,58-60$ & 613.08 & 343.2 & 83.6 & 0.195 & 25 & 182.5 & 50.4 & 0.328 & $\mathrm{~N}$ \\
\hline $17-5,18-20$ & 614.18 & 41.1 & 53.6 & 0.217 & 25 & 163.4 & 48.3 & 0.574 & $\mathrm{~N}$ \\
\hline $18-1,57-59$ & 617.57 & 129.3 & 44.8 & 0.175 & 25 & 144.3 & 75.3 & 0.252 & $\mathrm{~N}$ \\
\hline $18-2,59-61$ & 619.07 & 303.4 & 50.8 & 0.221 & 25 & 293.6 & 31.7 & 0.147 & $\mathrm{~N}$ \\
\hline $18-3,92-94$ & 620.92 & 139.0 & 61.7 & 60.619 & 25 & 144.0 & 60.8 & 54.013 & $\mathrm{~N}$ \\
\hline $18-4,54-56$ & 622.04 & 312.3 & 53.4 & 32.996 & 150 & 314.1 & 42.4 & 7.592 & $\mathrm{~N}$ \\
\hline $19-4,16-18$ & 634.86 & 178.3 & 62.7 & 1.872 & so & 203.6 & 51.7 & 0.782 & $\mathrm{~N}$ \\
\hline $20-1,31-33$ & 635.31 & 284.5 & 63.7 & 74.019 & 100 & 259.3 & 48.8 & 19.746 & $\mathrm{~N}$ \\
\hline $20-2,59-60$ & 637.09 & 292.0 & 48.6 & 0.285 & 50 & 269.4 & 41.9 & 0.303 & $\mathrm{~N}$ \\
\hline $20-4,25-26$ & 639.75 & 13.1 & 50.2 & 14.291 & 50 & 16.1 & 48.2 & 10.490 & $\mathrm{~N}$ \\
\hline $20-5,52-54$ & 641.52 & 81.2 & 53.9 & 1.722 & so & 80.2 & 58.3 & 1.350 & $\mathrm{~N}$ \\
\hline $27-1,124-127$ & 645.25 & 243.7 & 57.4 & 1.107 & NRM & & & & $\mathrm{N}$ \\
\hline $21-2,34-37$ & 645.84 & 127.1 & 53.3 & 4.880 & 50 & 128.2 & 46.7 & 4.899 & $\mathrm{~N}$ \\
\hline $21-3,22-25$ & 647.22 & 290.7 & 51.6 & 2.560 & 50 & 284.2 & 52.3 & 2.221 & $\mathrm{~N}$ \\
\hline $21-4,31-34$ & 648.81 & 235.4 & 55.3 & 2.431 & 50 & 245.4 & 41.2 & 2.193 & $\mathrm{~N}$ \\
\hline $21-6,27-30$ & 651.77 & 293.3 & 46.8 & 5.614 & 50 & 294.6 & 48.8 & 5.280 & $\mathrm{~N}$ \\
\hline $22-1,28-31$ & 653.28 & 318.2 & 59.0 & 3.409 & 50 & 80.2 & 58.3 & 1.350 & $\mathrm{~N}$ \\
\hline $22-1,121-123$ & 654.21 & 132.4 & 39.4 & 0.553 & 50 & 266.1 & 43.0 & 0.649 & $\mathrm{~N}$ \\
\hline $22-2,33-35$ & 654.83 & 244.5 & 42.9 & 0.783 & 50 & 266.1 & 43.0 & 0.649 & $\mathrm{~N}$ \\
\hline $22-2,107-109$ & 655.57 & 263.4 & 40.0 & 1.458 & so & 255.6 & 49.5 & 1.766 & $\mathrm{~N}$ \\
\hline $22-3,20-23$ & 656.20 & 263.2 & 60.9 & 7.952 & 50 & 267.1 & 51.5 & 4.711 & $\mathbf{N}$ \\
\hline $22-3,99-101$ & 656.99 & 202.5 & 48.2 & 4.362 & 100 & 197.7 & 47.8 & 4.383 & $\mathrm{~N}$ \\
\hline $22-5,38-40$ & 659.38 & 163.8 & 41.1 & 5.018 & so & 166.7 & 44.7 & 5.494 & $\mathrm{~N}$ \\
\hline $22-5,107-116$ & 660.07 & 352.6 & 52.8 & 13.136 & 100 & 327.8 & 55.7 & 5.707 & $\mathrm{~N}$ \\
\hline $22-6,7-10$ & 660.57 & 291.0 & 40.7 & 3.430 & 50 & 284.9 & 32.1 & 2.084 & $\mathrm{~N}$ \\
\hline $22-6,135-140$ & 661.88 & 20.1 & 44.8 & 1.857 & 100 & 16.7 & 44.5 & 2.244 & $\mathrm{~N}$ \\
\hline $23-1,8-10$ & 662.08 & 236.9 & 54.1 & 31.172 & 150 & 223.1 & 37.7 & 9.007 & $\mathrm{~N}$ \\
\hline $23-1,105-108$ & 663.05 & 274.4 & -85.3 & 101.46 & 50 & 193.4 & -88.4 & 53.134 & $\mathbf{R}$ \\
\hline $23-2,14-17$ & 663.44 & 0.2 & 50.8 & 30.805 & 150 & 9.5 & 44.1 & 7.406 & $\mathrm{~N}$ \\
\hline $23-2,92-94$ & 664.42 & 48.5 & 55.7 & 16.779 & 50 & 19.6 & 49.4 & 11.094 & $\mathrm{~N}$ \\
\hline $23-3,89-92$ & 665.89 & 261.1 & -57.6 & 39.136 & 50 & 254.9 & -53.4 & 27.732 & $\mathbf{R}$ \\
\hline $24-1,91-93$ & 671.91 & 102.3 & 60.5 & 101.733 & 50 & 112.7 & 58.5 & 61.812 & $\mathrm{~N}$ \\
\hline $24-2,88-90$ & 673.38 & 54.2 & 45.7 & 125.303 & 150 & 53.8 & 30.2 & 24.880 & $\mathrm{~N}$ \\
\hline $24-3,38-40$ & 674.38 & 210.0 & 52.9 & 120.167 & 800 & 274.6 & 11.0 & 3.469 & $\mathrm{~N}$ \\
\hline $25-1,29-31$ & 680.29 & 48.2 & 45.6 & 162.950 & 200 & 49.7 & 33.0 & 23.778 & $\mathrm{~N}$ \\
\hline $25-2,122-125$ & 682.72 & 49.1 & 45.2 & 263.152 & 500 & 332.2 & -4.0 & 6.816 & $\mathbf{R}$ \\
\hline $25-3,27-29$ & 683.27 & 291.3 & 42.5 & 236.584 & 500 & 350.4 & 45.0 & 3.664 & $\mathrm{~N}$ \\
\hline $25-1,49-51$ & & 297.5 & 49.1 & 1217.50 & NRM & & & & \\
\hline $29-1,81-83$ & & 268.9 & 56.8 & 1638.13 & NRM & & & & \\
\hline
\end{tabular}

Note: Symbols and abbreviations as in Table 1. 

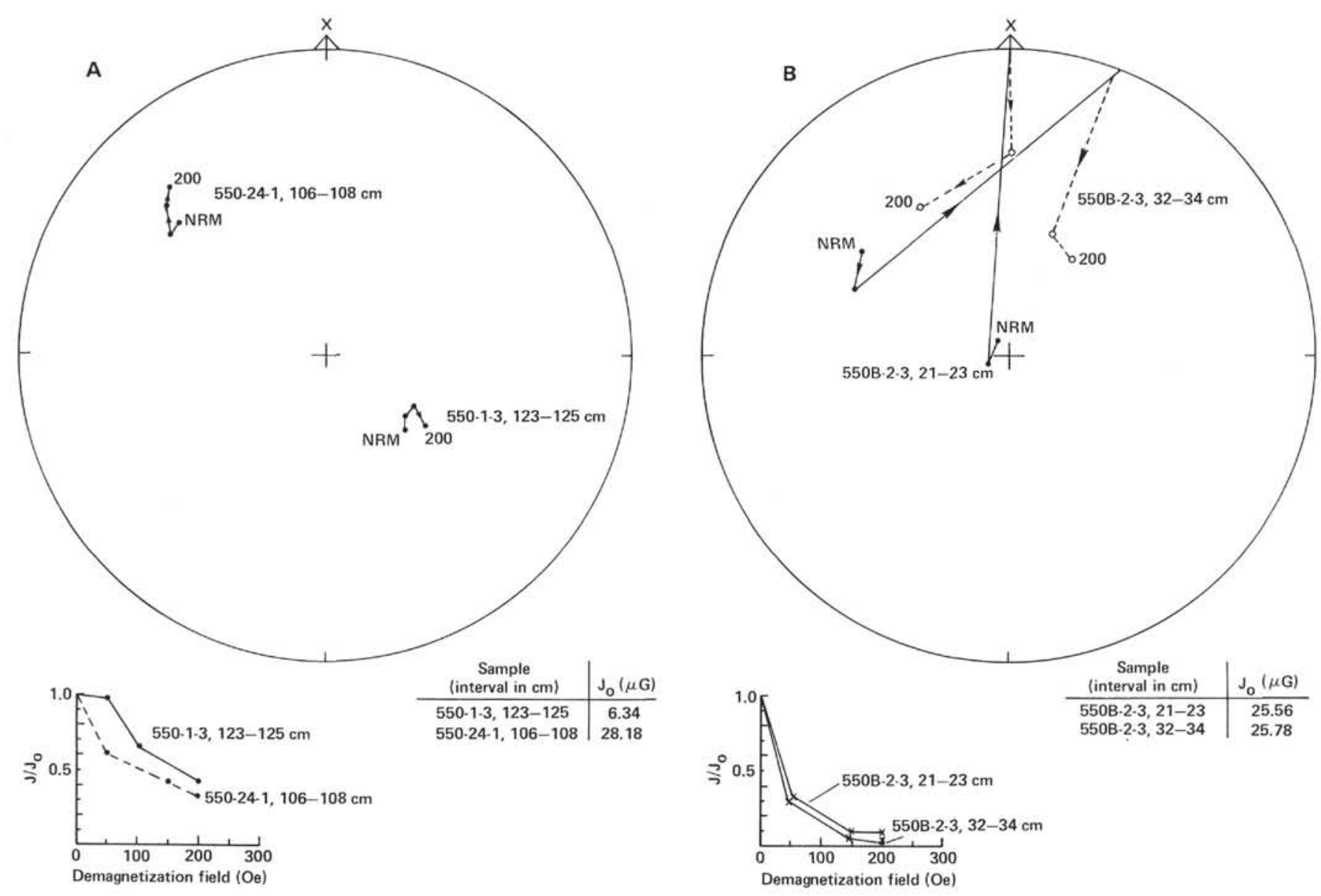

Figure 15. The response of representative samples from Site 550 to af demagnetization. A. Samples from Hole 550. B. Samples from Hole 550B. Symbols as in Fig. 2. 


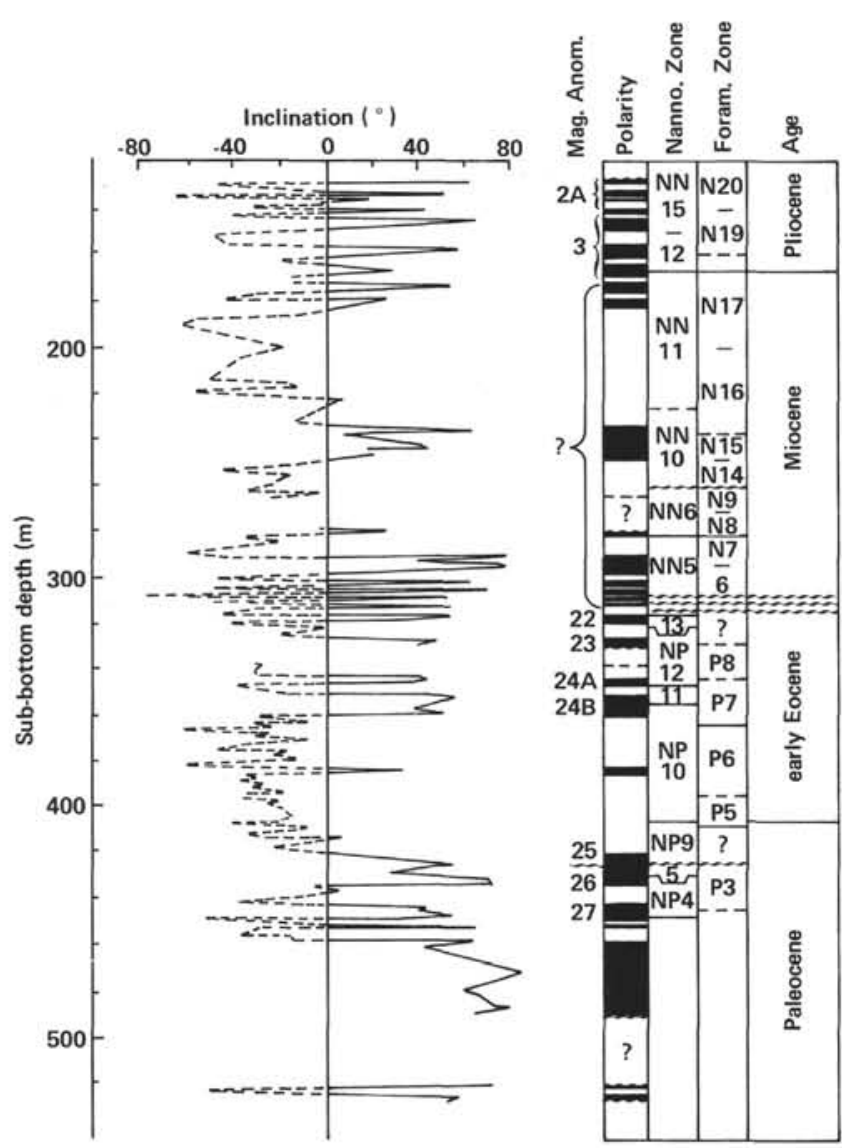

Figure 16. Downhole variation of magnetic inclination and the inferred polarity reversal sequence for Hole 550 .
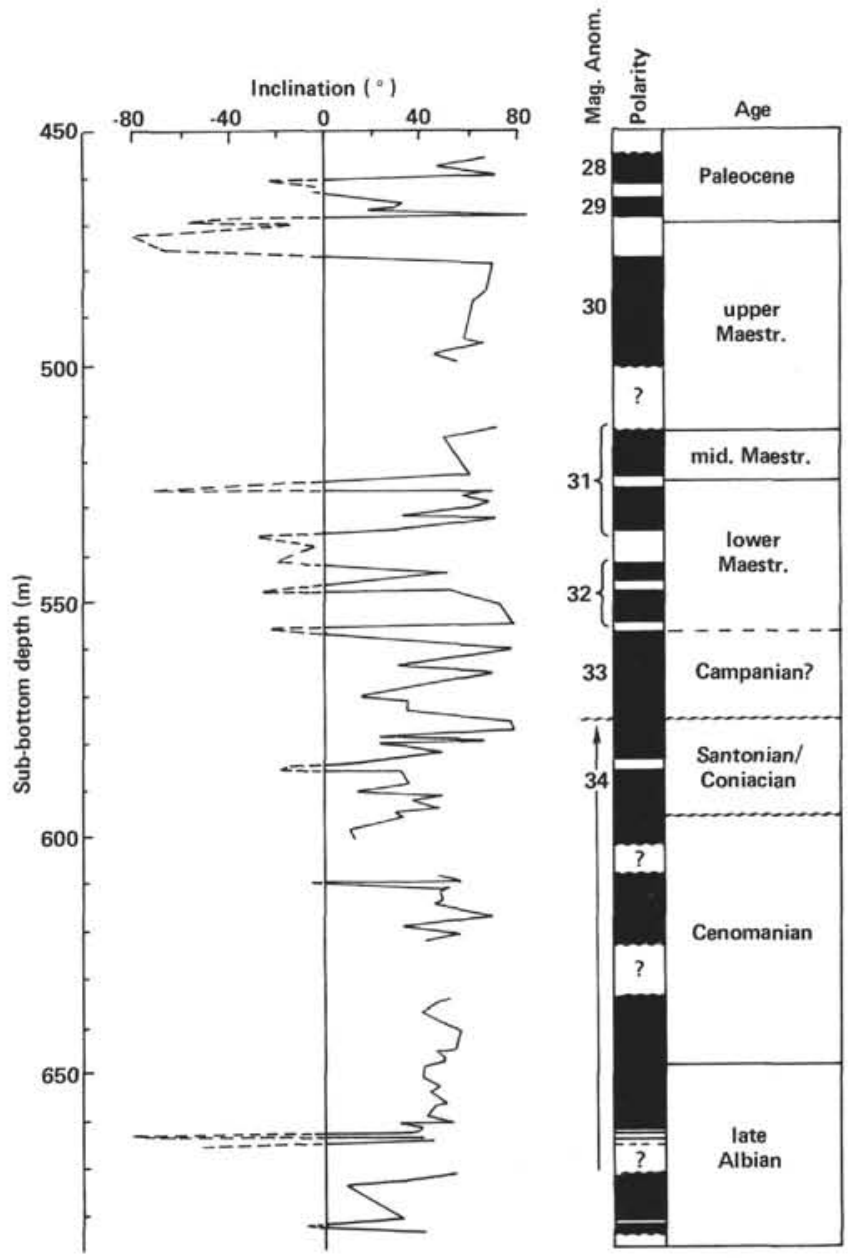

Figure 17. Downhole variation of magnetic inclination and the inferred polarity reversal sequence for Hole 550B.

Table 5. Paleomagnetic results, Hole 551.

\begin{tabular}{|c|c|c|c|c|c|c|c|c|c|}
\hline \multirow[b]{2}{*}{$\begin{array}{c}\text { Core-Section } \\
\text { (interval in } \mathrm{cm} \text { ) }\end{array}$} & \multirow{2}{*}{$\begin{array}{l}\text { Sub-bottom } \\
\text { depth } \\
\text { (m) }\end{array}$} & \multicolumn{3}{|c|}{$\begin{array}{c}\text { Before } \\
\text { demagnetization }\end{array}$} & \multirow{2}{*}{$\begin{array}{l}\text { Demagnetization } \\
\text { field } \\
(\mathrm{Oe})\end{array}$} & \multicolumn{4}{|c|}{$\begin{array}{c}\text { After } \\
\text { demagnetization }\end{array}$} \\
\hline & & $\begin{array}{l}\mathrm{D} \\
\left({ }^{\circ}\right)\end{array}$ & $\begin{array}{l}1 \\
\left({ }^{\circ}\right)\end{array}$ & $\begin{array}{l}\text { Int } \\
(\mu \mathrm{G})\end{array}$ & & $\begin{array}{l}\text { D } \\
\left({ }^{\circ}\right)\end{array}$ & $\begin{array}{c}1 \\
\left({ }^{\circ}\right)\end{array}$ & $\begin{array}{l}\text { Int } \\
(\mu \mathrm{G})\end{array}$ & Polarity \\
\hline $2-2,27-29$ & 105.77 & 323.3 & 37.0 & 7.941 & 500 & 300.9 & -8.2 & 0.519 & $\mathbf{R}$ \\
\hline $2-3,92-94$ & 107.92 & 109.0 & 68.8 & 9.702 & 300 & 131.3 & 40.0 & 1.341 & $\mathrm{~N}$ \\
\hline $3-1,56-58$ & 114.06 & 320.3 & 41.4 & 9.219 & 500 & 321.3 & 4.7 & 0.274 & $\mathrm{R}$ ? \\
\hline $3-2,31-34$ & 115.31 & 155.0 & 54.6 & 10.64 & 500 & 210.9 & -1.9 & 0.518 & $\mathrm{R}$ \\
\hline $3-3,24-26$ & 116.74 & 15.3 & 80.6 & 15.39 & 500 & 346.1 & 51.6 & 0.703 & $\mathrm{~N}$ \\
\hline $4-1,44-46$ & 123.44 & 16.1 & 45.8 & 20.96 & 300 & 42.0 & 18.3 & 0.549 & ? \\
\hline $4-2,83-85$ & 125.33 & 28.7 & 30.1 & 11.90 & 300 & 339.7 & 24.2 & 0.395 & $\mathrm{~N}$ \\
\hline $4-3,89-91$ & 126.89 & 218.2 & 62.7 & 16.697 & 300 & 227.5 & 20.0 & 1.630 & $\mathrm{~N}$ \\
\hline $4-4,80-82$ & 128.30 & 317.5 & 45.4 & 17.8 & 600 & 287.4 & 28.5 & 0.94 & $\mathrm{~N}$ \\
\hline $5-1,20-22$ & 132.70 & 54.3 & 72.6 & 2.93 & 100 & 310.6 & -34.9 & 0.59 & R \\
\hline $5-2,27-29$ & 134.27 & 38.4 & -1.8 & 0.30 & 75 & 22.3 & -8.9 & 0.207 & $\mathbf{R}$ \\
\hline $5-2,58-61$ & 139.08 & 286.5 & 80.2 & 0.41 & 75 & 196.7 & 62.6 & 0.18 & $\mathrm{~N}$ \\
\hline $6-2,141-143$ & 141.41 & 257.5 & 37.7 & 0.97 & 100 & 266.5 & 37.4 & 0.47 & $\mathrm{~N}$ \\
\hline $6-3,72-75$ & 142.22 & 140.7 & -16.6 & 4.179 & 100 & 112.9 & -63.2 & 14.70 & $\mathbf{R}$ \\
\hline
\end{tabular}

Note: Symbols and abbreviations as in Table 1. 


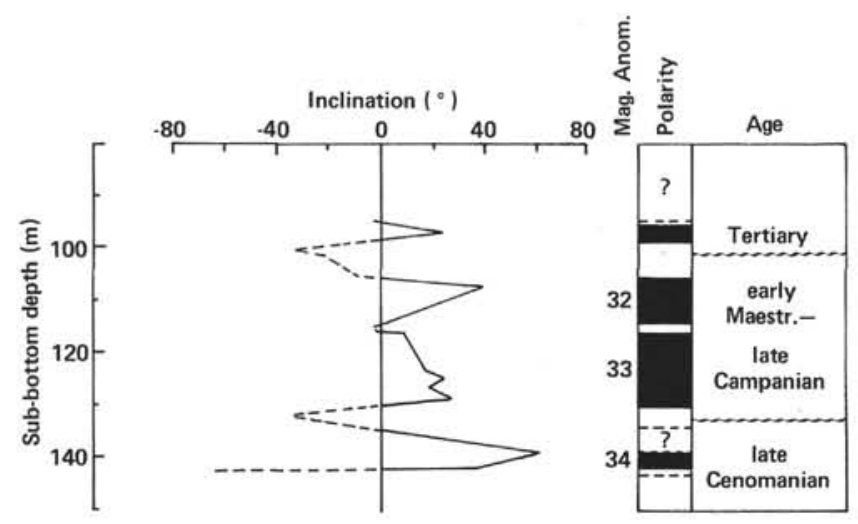

Figure 18. Downhole variation of magnetic inclination and the inferred polarity reversal sequence, Site 551 .

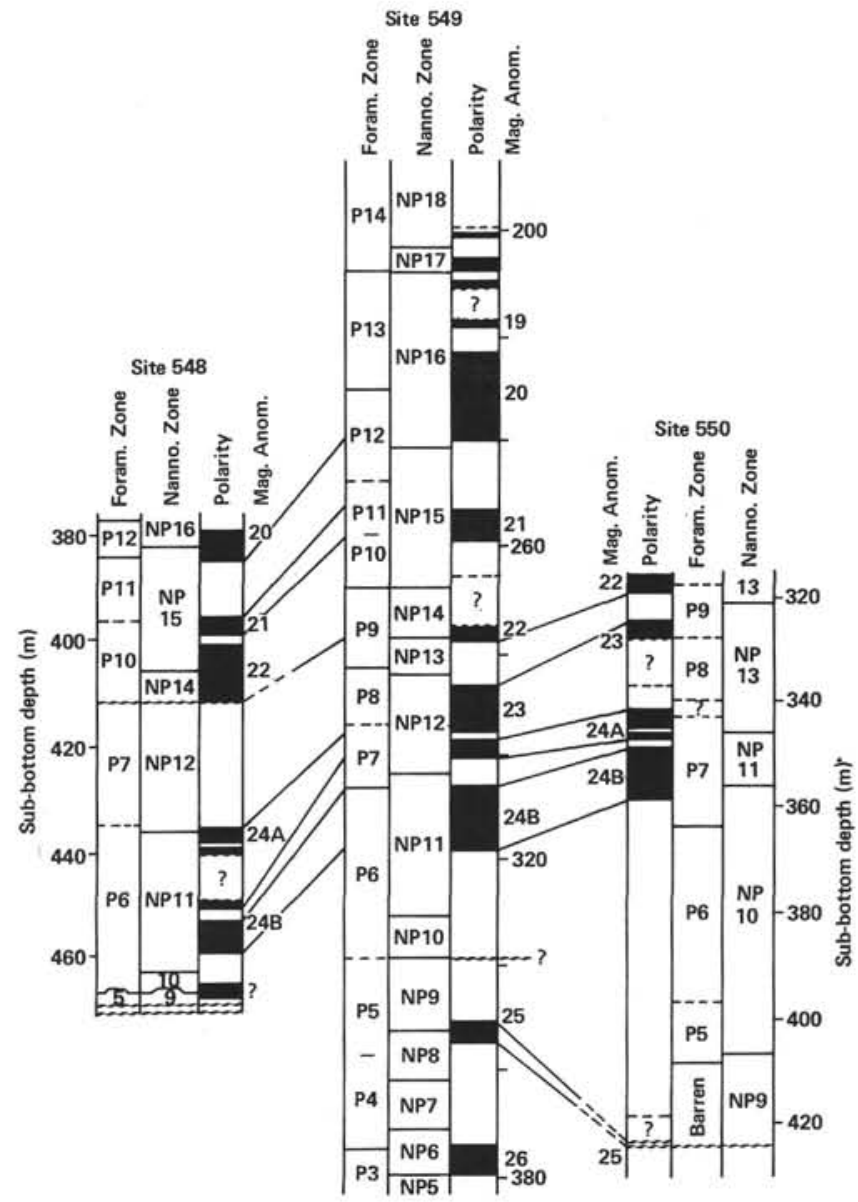

Figure 19. Magnetostratigraphic correlation of the middle Eocene to upper Paleocene sediments from Sites 548, 549, and 550. 